Chapter 11

\title{
Reconstitution and Physiological Protein Translocation Processes
}

\author{
Abol-Hassan Etémadi
}

\section{INTRODUCTION}

Two interpretations can be attached to the word "reconstitution." In a restricted sense, it generally refers to incorporation of proteins or proteinaceous complexes into bilayer membranes, often with the aim of restoring the natural function of the incorporated material. In a more general sense it refers to the in vitro rebuilding or regenerating of natural biological processes with or without involvement of membranes (Etémadi, 1985). With regard to protein translocation processes, reconstitution experiments in both the restricted and the general senses have been attempted.

Interest in protein translocation processes stemmed from studies on protein secretion, which culminated in the hypothesis of "vectorial transport" by Palade (1975). It was suggested that this process occurs cotranslationally and involves a "signaling device" at the $\mathrm{N}$ termini of precursors of the proteins (Milstein et al., 1972). This device is now referred to as the signal sequence (Blobel and Dobberstein, 1975). It had previously been proposed that secretory proteins crossed the hydrophobic core of the membrane through a channel (Redman and Sabatini, 1966). The "signal hypothesis" proposed formation of a proteinaceous transient channel or tunnel through which secretory proteins

Abol-Hassan Etémadi Laboratory of Biochemistry and Molecular Biology of Bacterial Lipids, Faculty of Sciences of the University of Paris VI, Paris, France. 

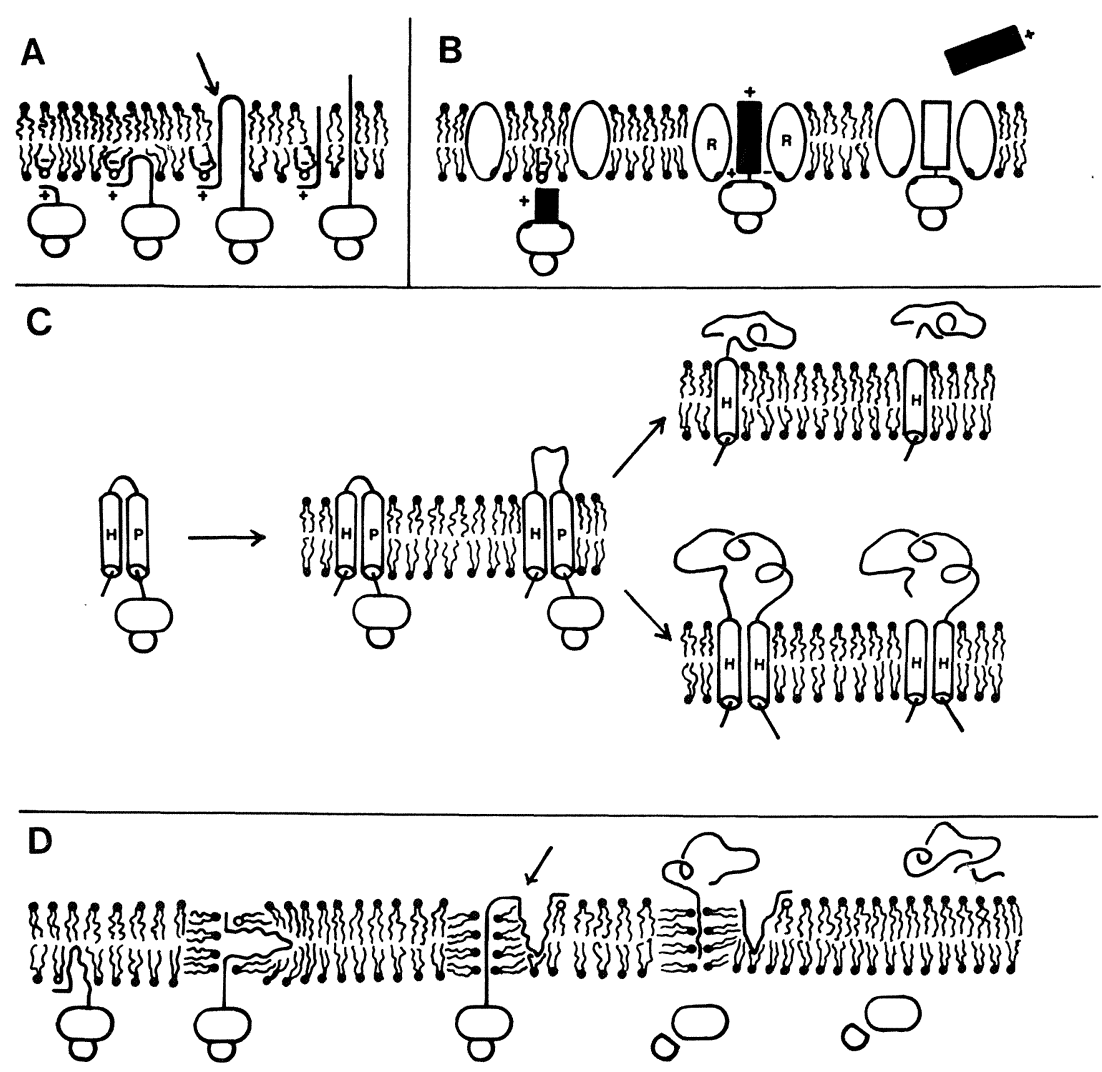

FIGURE 1. Some models for membrane-protein interaction during translocation of secretory and/ or transmembrane integral proteins are reproduced. In all schemes, unless otherwise stated, the lower side and the upper side represent the cytoplasmic (cis) and the extracytoplasmic (trans) compartment, respectively. In the loop model A (DiRienzo et al. 1978; Inouye and Halegoua 1980), positively charged amino acid(s) at the $\mathrm{N}$ terminus of the signal sequence react with negatively charged head groups of membrane phospholipids. The hydrophobic part of the signal sequence would interact with and loop back in the hydrophobic core of the membrane, bending being due to the presence of a glycine or proline residue in the hydrophobic segment of the signal. Chain elongation would continue until the cleavage site reaches the side remote from the cytoplasm where processing occurs. In model B (Garnier et al., 1980) the signal sequence as a double amphipathic structure inserts in the membrane. Its hydrophobic core has an $\alpha$-helical or extended conformation and its $\mathrm{N}$ terminus is extracytoplasmic. Only after this insertion ribophorin $(\mathrm{R})$ assembly around the signal peptide occurs and interaction of ribophorins, with the ribosomes, leads to the formation of a channel through which the protein is translocated. In the helical hairpin model, $\mathrm{C}$ (Engelman and Steitz, 1981), the signal sequence and the $\mathrm{N}$-terminal segment of the mature part of a nascent secretory or transmembrane integral protein form a hydrophobic $(\mathrm{H})$ and a polar $(\mathrm{P})$ helix, respectively, which together exhibit a helical hairpin conformation. The "helical hairpin" is inserted into the membrane in such a way that the $\mathrm{N}$ terminus of the signal sequence is cytoplasmic, as in the loop model. Continuation of translation extrudes residues of the polar helix in the trans side, while 
would pass in the temporally restricted duration of their translation (Blobel and Dobberstein, 1975). It was soon realized that early stages of membrane insertion of a transmembrane integral protein, the polypeptide chain of the glycoprotein of the vesicular stomatitis virus ( $G$ protein), were similar to those of translocation of secretory proteins and that a "stop transfer sequence" abrogated the translocation through the microsomal membrane, allowing membrane anchoring (Lingappa et al., 1978; Rothman and Lenard, 1977). In a more recent version of the signal hypothesis, the "topogenic sequence hypothesis," emphasis is placed on specific sequences of precursor proteins: signal sequences, stop transfer sequences, insertion sequences, and sorting sequences; the interaction of these sequences with specific membrane receptors would trigger translocation and intracellular transport processes (Blobel, 1980). In contrast with the signal hypothesis, some authors envisaged a post-translational translocation process for membrane proteins (Bretscher, 1973; Singer, 1977) and the "membrane trigger hypothesis" proposed the post-translational process for secretion of secretory proteins and membrane insertion of membrane proteins (Wickner, 1979).

Involvement or noninvolvement of membrane proteins and/or the mode of their involvement in protein translocation processes constituted one essential point of divergence between various models and hpyotheses suggested concomitantly with or since the above proposals. Different authors noted the hydrophobicity and the potential richness in secondary structure of prepeptides of secretory and membrane proteins (Austen, 1979; Burstein and Schechter, 1978; Engleman and Steitz, 1981; Garnier et al., 1980; Nesmeyanova, 1982; Palmiter et al., 1978; Rosenblatt et al., 1980; Thibodeau et al., 1978; Von Heijne and Blomberg, 1979) and suggested their interaction with membrane lipids. However, the process of translocation was integrated into various models, some of which are shown in Figure 1. DiRienzo et al. (1978) and Inouye and Halegoua (1980) proposed the "loop model" (Figure 1A) in which the translocation occurs cotranslationally but does not involve membrane proteins (see legend to

the polar helix is constantly completed by new residues reaching the membrane. If a secretory protein is being synthesized (upper part of model C), the mature chain is released after cleavage of the signal sequence which remains membrane inserted, as in the loop model. If, however, a transmembrane protein is being synthesized (lower part of model C), a second hydrophobic helix would be formed, which anchors the protein in the membrane. Continuation of the synthesis leads to the cytoplasmic localization of the C terminus of the molecule. Model D (Nesmeyanova, 1982) admits the initial electrostatic interaction of positive charges in the signal sequence with negatively charged head groups of membrane phospholipids as in the loop model. Then this model diverges from all others (see, however, aspects in Garnier et al. 1980) in admitting a phase change in the membrane and translocation of phospholipid molecules concomitantly wtih translocation of a secretory protein. The signal sequence after being cleaved (arrow) remains in the membrane. Its $\mathrm{N}$ terminus is here extracytoplasmic as well as its $\mathrm{C}$ terminus. 
Figure 1). The $\mathrm{N}$-terminal segment of the signal sequence is cytoplasmic and, after cleavage, the signal peptide remains bound to the membrane, while in the signal hypothesis of Blobel and Dobberstein (1975), designated the "linear model" (DiRienzo et al., 1978), it is released in the extracytoplasmic compartment. Austen (1979) admitted an electrostatic interaction with membrane lipids similar to the one suggested by the loop model. However, he suggested the participation of the signal sequence itself and other proteins as channel formers in the process of translocation, while Garnier et al. (1980) (Figure 1B) proposed the interaction of the signal sequence as a double amphipathic structure with membrane lipids. Ribophorins, two transmembrane glycoproteins the presence of which in rough microsomes was previously reported (Kreibich et $a l ., 1978 \mathrm{a}, \mathrm{b})$, would then be recruited to form a channel for translocation. No other protein would be involved. In this hypothesis the signal sequence is released in the extracytoplasmic compartment. Von Heijne and Blomberg (1979) and Von Heijne (1980) proposed, in a model designated "direct transfer model" and resembling the loop model, the membrane insertion of the signal sequence of nascent chains as two antiparallel helices, connected by a short loop. It was considered, however, that ribosomes would bind to the membrane presumably through ribophorins, but that ribophorins are not involved in formation of a tunnel; their interaction with ribosomes would be strong enough to force the entry during the translation of hydrophilic residues of the nascent chain into the hydrophobic phase of the membrane. Nevertheless, passage of a sufficiently charged and hydrophilic segment through the hydrophobic core would be so energetically unfavorable that the ribosome would detach from the membrane and the $\mathrm{C}$ terminus of the molecule would be translated and remain in the cytoplasm. A transmembrane protein would thus be generated. In another model, suggested by Engleman and Steitz (1981) and designated the "helical hairpin model" (Figure 1C), the $\mathrm{N}$ terminus of the nascent chain inserts into the membrane as two antiparallel helices. In this hypothesis no membrane proteins acting as receptors or translocators are involved, even a ribosome-membrane interaction is not envisaged. What plays the role of a stop transfer sequence is the hydrophobic segment, as in the "topogenic sequence hypothesis." However, here no membrane protein is involved in interaction with this sequence.

In another model, proposed by Nesmeyanova (1982) (Figure 1D), the secretion of proteins (through the bacterial cytoplasmic membrane) is proposed to occur in a process coupled with translocation of phospholipid molecules. Passage of the hydrophilic exported proteins occurs through a hydrophilic channel formed by phospholipid head groups, as a result of bilayer to hexagonal type II change of the membrane structure. Here again, no proteinaceous receptor-translocator is required. No energy additional to that used for synthetic processes is dissipated. 
Thus, apart from the "membrane trigger hypothesis," all the models for translocation of secretory and transmembrane integral proteins envisage a cotranslational process. This is quite legitimate, particularly in the case of eukaryotic cells, and remains essentially valid, although as we shall see, recent interesting evidence shows the feasibility of a post-translational translocation of some of these proteins under particular experimental conditions. In fact, the presence of free and membrane-bound ribosomes in homogenates of eukaryotic cells has long been reported. Bound ribosomes are attached to the rough endoplasmic reticulum (see Leskes et al., 1971a,b; Palade and Siekevitz, 1956a,b), either loosely or tightly (Adelman et al., 1973), that is, they are detachable by high salt alone or requiring in addition puromycin treatment. Tightly bound ribosomes are linked to the endoplasmic reticulum through an electrostatic linkage, presumably to ribophorins (Kreibich et al., 1978a,b, 1980; see Section 2.1), in addition to being linked through nascent chains. Several studies indicate involvement of ribosomes bound to the endoplasmic reticulum in the genesis of secretory and transmembrane integral proteins. Binding of ribosomes involved in the genesis of these proteins occurs early in protein translation (see Blobel and Dobberstein, 1975; Boime et al., 1977; Burr and Burr, 1981; Eskridge and Schields, 1982; Meek et al., 1982; Scheele et al., 1980).

In the case of bacterial exported proteins, involvement of ribosomes bound to the inner membrane (Cancedda and Schlesinger, 1974; Inouye and Beckwith, 1977; Lory et al., 1983; Randall and Hardy, 1977; Randall et al., 1978; Rasmussen and Bassford, 1985; Smith et al., 1981) was also observed. However, bacterial-bound ribosomes involved in translation of exported proteins could be released by action of puromycin alone, with no requirement for high salt (Davis and Tai, 1980; Smith et al., 1978a,b, 1979). Thus, despite the proximity of ribosomes to the bacterial membrane (Davis and Tai, 1980) no electrostatic binding could be demonstrated in this case. Some genetic work once suggested involvement of bacterial ribosomes in cotranslational export of outer membrane and periplasmic proteins (Emr and Bassford, 1982; Emr et al., 1981). However, other work could not confirm this proposal (see Ito et al., 1984; Shiba et al., 1984).

With regard to proteins translocated to mitochondria (see Chua and Schmidt, 1979; Neupert and Schatz, 1981), chloroplasts (Chua and Schmidt, 1979; Grossman et al., 1980; Highfield and Ellis, 1978), and microbodies (Lazarow and De Duve, 1973; Lazarow et al., 1982), it is generally admitted that they are predominantly synthesized on free ribosomes. In some cases the contribution of bound ribosomes, in addition to free ribosomes, is reported in the genesis of these proteins. Organellar proteins of mitochondria, chloroplasts, and microbodies, synthesized under the direction of nuclear genome and translocated into the respective organelle by passage through at least one membrane, 
can generally be post-translationally translocated even in cases where bound ribosomes were reported as contributors in their genesis. I proposed calling these proteins "migratory proteins"' (Etémadi, 1980a, 1985). Some migratory proteins in chloroplasts must cross through three membranes to reach their ultimate destination (Figure 2).

Besides the involvement or noninvolvement of proteinaceous material in the protein translocation processes and their co- or post-translational nature, the



FIGURE 2. This figure shows different compartments of a mitochondrion (upper drawing) and a chloroplast (lower drawing). In both cases, organelles are enclosed in an outer and an inner membrane (Om and $\mathrm{Im}$, respectively). The intermembrane space of the mitochondrion (i) and the matrix compartment (m) are shown. In the chloroplast, an intermembrane space (i) and a stroma compartment (s) are recognized. The inner membrane of mitochondria presents expansions plunging into the matrix; these are cristae (C). In the case of chloroplasts, two types of membrane structure exist in the stroma. These are stroma thylakoids (ST) and grana thylakoids (GT), the last being formed of stacked thylakoid membranes. Thylakoids enclose a single intrathylakoid compartment (it), due to the presence of interconnections. For a protein to reach this compartment it should traverse three membrane bilayers. 
energy requirement remained an undecided point. The first clue as to the energy dependency of protein translocation came from studies in the laboratory of Neupert and his colleagues and Schatz and his collaborators on mitochondrial proteins of Neurospora crassa (Hallermeyer and Neupert, 1976; Hallermeyer et al., 1977; Harmey et al., 1976, 1977) and Saccharomyces cerevisiae (Nelson et al., 1979; Schatz, 1979), respectively. In the case of mitochondrial proteins, demonstration of an energy requirement proved to be easier than with proteins translocated through the endoplasmic reticulum, since in the last case processes of protein translation and translocation were most generally inseparable, translocation taking place cotranslationally. Furthermore, exactly what the energy requirement meant was not clear. As soon as the energy requirement was known, as a result of work in Neupert's and Schatz's laboratories, I assimilated translocation of migratory proteins to "some sort of active transport" (Etémadi, 1980a) and felt that the energy requirement should be considered in a general framework, crucial elements of which would be involvement of proteinaceous receptor-translocator systems in organellar membrane and requirement for energy for the process of translocation itself. Soon after, Neupert and Schatz (1981) made a similar proposal. At that time a generalization to the case of secretory and transmembrane proteins translocated through microsomal membrane was not possible because of the cotranslational nature of the process. However, as pointed out (Etémadi, 1980a), while the significance of the energy requirement even for post-translational protein translocation was overlooked (e.g., see Blobel, 1980), emphasis was placed on the usefulness of gaining this knowledge if one is to have an understanding of the general problems of protein translocation (Etémadi, 1980a). Recent progress, as a result of work in different laboratories (Hansen et al., 1986; Rothblatt and Meyer, 1986b; Waters and Blobel, 1986) indicates the feasibility of a post-translational energy-dependent protein translocation through the microsomal membrane. The report by Maher and Singer (1986) of the possibility of post-translational translocation through this membrane of a protein reputed to be co-translationally translocated would have constituted the best support for this process. However, the evidence was recently questioned by Ibrahimi (1987), see below. Nevertheless, recent advances allow an extension to the case of secretory and transmembrane proteins of the proposal mentioned above concerning migratory proteins (Etemadi, 1980a, 1985 ) in stating that translocation of these proteins also involves "some sort of active transport"' (Etemadi, 1986a, 1987, 1988).

The whole problem of physiological protein translocation can now be envisaged at two levels: (1) that of the general mechanism, which, with the exception of some specific cases, seems to involve "active transport," and (2) that of mechanistic details dealing with the steps and molecular events concerning the interaction of precursors and receptor-translocators, the exact path of 
proteins during translocation, the co- or post-translational occurrence and/or feasibility of translocation, and the way energy consumption is coupled to the above events.

Reconstitution in both the general and restricted sense has been attempted, and the most useful information to date has been derived from the first type of experiment; the path is now paved for fruitful liposomal reconstitution assays. The limited liposomal experiments made in the past were generally vitiated, since they relied mostly on the thermodynamics of protein-liposome interaction, ignoring involvement of receptor-translocators or the energy requirement. In what follows I first go through what we presently know of different aspects of physiological protein translocation processes. This allows a comparison to be made with assays using liposomal systems. Ultimately, a recent "unitary hypothesis" suggested by Singer et al. (1987a,b) is discussed and prospects for principles guiding further liposomal reconstitution experiments are reformulated.

\section{MEMBRANE PROTEINS AND PROTEIN TRANSLOCATION PROCESSES}

\subsection{Microsomal Membrane Proteins}

Various observations based on the inhibitory effect of salt washing (Meyer and Dobberstein, 1980a,b; Warren and Dobberstein, 1978), mild proteolysis (Walter et al., 1979), or $N$-ethylmaleimide treatment (Katz et al., 1977) of microsomes indicated involvement of proteinaceous material in the process of protein export through the endoplasmic reticulum. At the onset, such experiments were aimed essentially at probing the eventual involvement of proteins as channel or tunnel formers for translocation. They led to the discovery of the signal recognition particle (SRP) and docking protein. SRP, an 11S nucleoprotein complex, formed of six protein subunits of 72,000,68,000, 54,000, 25,000, 14,000 , and $9000 \mathrm{Da}$ and an RNA molecule, identical to a cytoplasmic 7SL RNA, could be detached by salt wash of ribosomes (Walter and Blobel, 1980; Walter et al., 1981). A similar complex (of $M_{\mathrm{r}} 250,000 \mathrm{Da}$ ) was found to be present as a soluble component in reticulocyte lysates, while the wheat germ extracts were devoid of this material (Meyer et al., 1982).

During in vitro experiments on the precursor of a secretory protein (preprolactin) synthesized in the presence of wheat germ protein translation system, polysomes bind to the SRP and protein translation is stopped after a chain of 79 residues is synthesized (Meyer et al., 1982; Walter and Blobel, 1981a; Walter et al., 1981). Salt-washed microsomes that had lost their protein translocation capability, when added to such a translation-arrested system, released the 
translation arrest and recovered their translocation and processing aptitude (Walter and Blobel, 1981b). In other cases, protein translocation through (Bassuener et al., 1984; Erickson et al., 1983; Mueller et al., 1982) or insertion into (Anderson et al., 1982) the microsomal membrane was reported to be dependent on a signal recognition particle. The signal sequence was shown (Wiedmann et al., 1986a) to bind to the SRP, specifically to its 54,000 Da subunit as revealed by cross-linking experiments (Krieg et al., 1986; Kurzchalia et al., 1986; Wiedmann et al., 1987). More recent data (Siegel and Walter, 1988a) indicate that modification by $N$-ethylmaleimide of the 54,000 -dalton subunit prevents binding of nascent chains to SRP, while that of 68,000 - and 72,000-dalton subunits does not prevent the elongation arrest but binding to the docking protein and the subsequent translocation.

The exact significance of protein translation arrest by SRP is unclear, however. It was observed not to occur with some proteins (Anderson et al., 1982) that were devoid of a cleaved signal sequence. Nevertheless, it was observed in other cases of proteins with an uncleaved signal (Bonatti et al., 1984; Brown et al., 1984; Lipp and Dobberstein, 1986a; Rottier et al., 1985; Sakaguchi et al., 1984). Finally, a variability of arrest of protein synthesis, even with proteins endowed with a cleared signal, was observed (Siegel and Walter, 1985). Meyer (1985) reported that the arrest in translation is only observed with a wheat germ protein synthesizing system, not with reticulocyte lysates or a HeLacell-free system. Finally, association studies after dissociation of SRP revealed that omission of subunits of 9000 and 14,000 led to an incomplete particle, which, however, mediated translocation at a reduced level but without translation arrest. Thus, translation arrest is not required for translocation (Siegel and Walter, 1986).

Recently, Prehn et al. (1987) reported that the cotranslational translocation of precursor proteins of different origin [human placental lactogen, plant zein (a storage protein of protein bodies) and the bacterial plasmid $\beta$-lactamase] could occur through wheat germ microsomal membrane. A plant SRP-like complex was required. However, this particle not only did not arrest protein synthesis but increased the translation. This plant SRP did not reconstitute the translocation function of salt-washed canine microsomal membranes.

In canine SRP, the two smallest subunits of 9000 and 14,000 Da are bound to the $5^{\prime}$ and $3^{\prime}$ ends (Alu sequences) of the 7SL RNA, while other subunits are linked to the central (S) sequence (Guldenfinger et al., 1983). The most tightly bound subunit to the RNA appeared to be the 19,000 Da protein (Scoulica et al., 1987). Dissociation and reassembly studies indicated a functional role for the RNA part of the SRP complex in addition to its protein subunits (Walter and Blobel, 1983). In the case of yeast microsomes, despite the fact that neither the protease treatment nor the salt wash abrogates their translocation aptitude for yeast secretory proteins (Rothblatt and Meyer, 1986a), the 
presence of a postribosomal proteinaceous factor of $9.6 \mathrm{~S}$ devoid of RNA and involved in post-translational protein translocation of yeast pre-pro- $\alpha$-factor, was reported. The possibility that this complex may constitute a "denaturase", involved in unfolding protein precursors before they can be taken up posttranslationally was envisaged (Waters et al., 1986). The factor, however, wás more recently assimilated to an evolutionary precursor of SRP (Fecycz and Blobel, 1987). Thus, the function attributed to the RNA in SRP remains uncertain, since the yeast complex is devoid of RNA.

Another microsomal protein, involved in protein translocation is designated as "docking protein;"' it was described as an integral membrane protein of about 72,000 Da (Meyer et al., 1982a) involved in the release of the arrest in protein translation resulting from interaction of SRP with the signal sequence of the nascent chain (Gilmore et al., 1982; Meyer, 1982; Meyer et al., 1982). This protein shows a 20 -fold enrichment in the rough endoplasmic reticulum as compared to the smooth endoplasmic reticulum (Hortsch et al., 1985). Proteolysis of rough microsomes cleaves a fragment of approximately 60,000 Da from this protein (Meyer et al., 1982a,b). The length of the fragment of the protein cleaved by proteolysis of microsomes depends on the proteolytic enzyme used. A recent study indicated that elastase cleaves a fragment of 59,000 $\mathrm{Da}$, leaving a segment of $14,000 \mathrm{Da}$ anchored in the membrane, while trypsin releases a shorter $46,000-\mathrm{Da}$ fragment. The part involved in releasing of the arrest of protein translation is located in the segment of 13,000 Da, the difference between the fragments cleaved by the two enzymes (Hortsch et al., 1985). Lauffer et al. (1985), reported an $\mathrm{M}_{r}$ of 69,684 Da for docking protein, which is shown as a transmembrane protein anchored in the microsomal membrane through its $\mathrm{N}$ terminus. More recently, Tajima et al., (1986) obtained evidence for the docking protein to be composed, in addition to the 70,000-Da polypeptide just described, of another polypeptide chain of approximately 30,000. Hortsch and Meyer (1988) report now that this 30,000-Da $(\alpha)$ subunit of the docking protein might be involved in binding of the $72,000-\mathrm{Da}(\beta)$ subunit to the microsomal membrane. This binding would occur post-translationally without involvement of SRP and docking protein. The 72,000 dalton subunit would thus not be a transmembrane protein.

It is worthwhile at this point to consider two crucial aspects of the possible involvement of SRP and docking protein in the protein translocation process: (1) What is the bearing of their discovery on the co- or post-translational nature of microsomal protein translocation? (2) What are the molecular events accompanying and following the release of translation arrest and allowing translocation? With regard to the first point, the phenomenon of arrest of translation and its release in the presence of salt-washed microsomes appeared compatible with a strict coupling of translation with translocation. Then, when it was found that 
protein translation arrest does not occur in all cases, it was explicitly or implicitly admitted that protein translocation was still tightly associated with its translation. More recent experiments bring fundamental changes in this respect. Ainger and Meyer (1986) report that in the wheat germ protein translation system, SRP can still arrest translation if two-thirds of the nascent IgG light chain is completed, but that this chain can be translocated by addition of rough ribosomes. Experiments during which nascent bovine prolactin precursor was translocated led to a similar conclusion. A very recent report by Siegel and Walter (1988b) confirms these data and indicates that the discrepancy between these results and those reported above as to the length of nascent chains of pre-prolactin with which SRP can interact in a functional manner is correlated with different concentrations of SRP used. Thus, the possibility, under in vitro experimental conditions, of the lack of a strict coupling of translation with translocation is confirmed. As to the second point, neither the nascent chain nor the ribosome binds to the docking protein (Gilmore and Blobel, 1983). SRP is released and docking protein is present in microsomes in a far less stoichiometric ratio than bound ribosomes. Thus, it cannot apparently be directly involved in the actual translocation. An observation, the exact significance of which remains to be appreciated, is that in Xenopus oocytes expressing ovalbumin, neither the signal recognition particle nor the docking protein seemed to play a significant role in the synthesis and export of the protein (Richter et al., 1985). In any event, the question is raised as to the nature of protein (s) of the microsomal membrane directly involved in the binding and translocation processes. Observations on $S$. cerevisiae mutants defective in protein translocation led to the suggestion that microsomal proteins other than SRP and docking protein are also involved in protein translocation (Ferro-Novick et al., 1984a,b). However, further studies on this case did not allow the discovery of an additional transmembrane integral protein in protein translocation (Bernstein et al., 1985). Nevertheless, more recently isolation of a thermosensitive yeast mutant (sec 61) was reported that accumulates multiple secretory and vacuolar precursor proteins. These are bound to the endoplasmic reticulum but are not processed and have a large cytoplasmic exposure indicating that their translocation is not completed (Deshaies and Schekman, 1987). It would be interesting to learn more about the way the mutated gene is involved in the translocation process.

Ribophorins, as mentioned above, were proposed to be implicated in binding of ribosomes (Kreibich et al., 1978a,b); for a recent report on the structure and orientation of ribophorins see Crimaudo et al., 1987). Such a role for these proteins was subsequently questioned (Bielinska et al., 1979). However, further studies (Amar-Costesec et al., 1984; Kreibich et al., 1982; Marcantonio et al., 1984) supported their possible correlation with ribosome binding, though besides their direct electrostatic binding to ribosomes, alternatives such as their 
implication in binding of nascent chains to the membrane or the transfer of these chains to the luminal side were not excluded (Marcantonio et al., 1984; see also Harnik-Ort et al., 1987). Recent controlled proteolytic experiments (Hortsch et al., 1986) carried out on microsomes led to recognition of conditions under which some $85 \%$ of ribophorin I and $100 \%$ of ribophorin II had resisted degradation; nonetheless, these vesicles had lost their aptitude for binding ribosomes, even after the function of docking protein (which was also cleaved) was reconstituted. Thus, proteins other than ribophorins should be involved in binding ribosomes to the membrane, although their contribution to the process cannot be excluded.

Different reports suggest the possible existence of binding sites for the signal sequence on the microsomal membrane. In fact, chemically synthesized prepeptides of a secretory protein inhibited competitively the in vitro translocation through microsomes of various secretory proteins (Majzoub et al., 1980; Rosenblatt et al., 1979). Interestingly, precursors of secretory proteins could bind to salt-washed microsomal membrane (Prehn et al., 1980, 1981). Studies by Austen and his colleagues (Austen and Ridd, 1983; Austen et al., 1984) show the binding to salt-washed microsomes of a synthetic peptide representative of signal sequences of precursors of secretory and transmembrane integral proteins. This binding inhibited translocation of secretory proteins. The synthetic peptide did not bind to the signal recognition particle. This point seems at variance, however, with a report by Duong et al. (1987), which does not exclude interaction of synthetic prepeptides with SRP. In any event, a recent report by Robinson et al. (1987) implicates a microsomal membrane protein of approximately 45,000 $\mathrm{Da}$, distinct from all proteinaceous components mentioned above and from the signal peptidase, in binding of signal sequences to the microsomal membrane. Other authors, however, had suggested the possibility that the microsomal signal peptidase could constitute or be part of the translocation system (Evans et al., 1986). Recently also Wiedmann et al. (1987) reported the presence of a microsomal integral membrane glycoprotein of 35,000 daltons, which was designated as signal sequence receptor, to which the signal sequence could bind after release from the docking protein. Let us mention that a report by Gilmore and Blobel (1985), indicating that during their translocation secretory proteins cross an aqueous environment, was interpreted as favoring the involvement of a proteinaceous channel. However, such an interpretation is not unexceptionable since an aqueous channel can also be formed by head groups of lipids, as is inherent in the model proposed by Nesmeyanova (1982) (see Figure 1D). In summary, despite the fact that there are several hints to the involvement of proteinaceous components in the process of microsomal protein translocation, further work is required to fully establish their nature and details of their function. 


\subsection{Bacterial Membrane Proteins}

Here again the inhibition of translocation of exported proteins by pronase treatment of insidè-out inner $E$. coli membrane vesicles revealed involvement of membrane proteins in the process (Rhoads et al., 1984; Smith, 1980). In other studies, compositional differences in protein content could be observed in ribosome-free membrane and membrane-ribosome complexes obtained from Bacillus subtilis (Caulfield et al., 1983; Horiuchi et al., 1983a,b; Marty-Mazars et al., 1983). Some proteins unique to the membrane-ribosome complex were thought to be involved in protein translocation. Further analysis by Caulfield $e t$ al., (1984) revealed that the membrane-ribosome complex could in fact be separated into two fractions, the first comprising monosomes and an $\mathrm{S}$ complex reminiscent of the signal recognition particle. This complex contained proteins of $64,000,60,000,41,000$ and 36,000 Da. However, S complex was not present in the soluble fraction, was devoid of RNA, and could not lead to translation arrest. The second fraction contained polysomes attached to the membrane. This fraction comprised only the 64,000-Da protein of the complex (Caulfield et al., 1984). S complex was thought to be involved in initiation of export. Its subunits, except the 64,000-Da entity, would detach upon contact of the protein-translating ribosome with the membrane; the complex would thus break up (Caulfield et al., 1985). That bacterial 6S RNA is not involved in protein translocation is confirmed by other authors (Lee et al., 1985). However, Muèller and Blobel (1984) reported the presence in $E$. coli homogenates of an "export factor' of $12 \mathrm{~S}$ and suggested the possibility that it may contain an RNA species smaller than the 6S RNA. This factor and the one from the postribosomal yeast fraction, the function of which it could mimic interchangeably, were considered as evolutionary precursors of SRP (Fecycz and Blobel, 1987). Adler and Arvidson (1987) found the presence of a multiprotein complex on membrane-bound ribosomes of Staphylococcus aureus, correlated with exoprotein synthesis, which contains four tightly bound polypeptides of $M_{\mathrm{r}} 71,000,60,000$, 46,000 , and 41,000 Da. The 60,000-Da entity was antigenically correlated with the 64,000-Da entity of $B$. subtilis mentioned above. However, the $S$. aureus complex is present both on membrane-bound ribosomes, where it is located between the membrane and ribosomes, and in solution. This complex is thus more similar to the microsomal SRP. However, it exhibits only four subunits and does not contain RNA.

Besides the above analytical data, other observations indicate the involvement of proteins in translocation of exported proteins through the bacterial cytoplasmic membrane. Studies on $E$. coli mutants defective in protein translocation (Wanner et al., 1979) and several reports on $E$. coli expressing fusion proteins led to the suggestion of the presence of proteinaceous export devices. 
In such assays the gene fusion concerns the $\mathrm{N}$ terminus of the precursor of an exportable protein, including the signal sequence, linked $\mathrm{N}$ terminally to a cytoplasmic protein such as $\beta$-galactosidase. Examination of localization of hybrid proteins 'expressed in $E$. coli and the inhibition of translocation of cellular exportable proteins as a result of jamming of the membrane are interpreted in terms of the existence of export machinery in the membrane (see Bassford $e t$ al., 1979; Emr and Silhavy, 1982; Emr et al., 1980; Ito et al., 1981; Kiino and Silhavy, 1984). Studies on conditional E. coli mutants defective in protein export led to the proposal of involvement of a gene (sec A gene) product of 92,000 Da in protein translocation (Oliver and Beckwith, 1981, 1982a,b). The protein was assimilated to a component of a SRP-like complex or to docking protein. Interaction with a defective translocation system led to the arrest in the synthesis of transported protein (Kumamoto et al., 1984). Also, the product of a sec B gene was involved in protein translocation in E. coli (Kumamoto and Beckwith, 1983). The product of this gene (Kumamoto and Beckwith, 1983) and that of another sec Y (or prl A) gene were suggested to interact with the product of sec A gene in the export machinery (Bickman et al., 1984). However, it was very recently reported (Collier et al., 1988) that sec B gene encodes a soluble cytoplasmic protein involved in unfolding of a periplasmic protein, maltose-binding protein, preventing its premature folding before translocation. The sec $\mathrm{Y}(\mathrm{prl} \mathrm{A})$ gene product is identified to an integral protein of the inner E. coli membrane (Akiyama and Ito, 1985, 1987; Ito, 1984). Involvement of still another gene (prl D gene) product as well as that of sec $\mathrm{Y}$ gene in the process of translocation could be supported by observation of $E$. coli suppressor mutations in these genes, allowing translocation of exported proteins showing defective signal sequences. Products of these two genes were suggested to be assembled in a complex (Bankaitis and Bassford, 1985a). Extragenic suppressor mutations also suggested involvement of sec A, prl A, sec $\mathrm{C}$, and other loci in protein translocation in $E$. coli which would possess a general mechanism of coupling of synthesis of exported proteins with secretion (Oliver, 1985; Oliver and Liss, 1985). Both prl A and sec A gene products were implicated in the indirect binding of ribosomes involved in the synthesis of exported proteins to the membrane (Rasmussen and Bassford, 1985). More recently, however, it was found difficult to identify components of export machinery by suppression of sec A mutations (Lee and Beckwith, 1986), and Strauch et al. (1986) could not confirm coupling between synthesis and translocation of exported proteins. For example, inhibition of the synthesis of maltose-binding protein in sec A (am) mutants was correlated with cAMP depletion resulting in a decreased transcription of mal $\mathrm{E}$ gene. Addition of cAMP led to the synthesis of the protein while translocation remained blocked. In addition, sec A (am) mutation did not affect synthesis of OmpA protein and lipoprotein, two other exported proteins. Furthermore, Shiba et al. (1986) find that the 
protein export defect as a result of sec Y 24 (ts) mutation can be suppressed extragenically by numerous (at least six) genes, they consequently draw attention to the unlikeliness that all these genes would specify components of the translocation system. In addition, indications suggest that sec $\mathrm{Y}$ gene product might be involved in post-translational translocation of an outer membrane protein, OmpA protein, not in coupling synthesis and translocation (Bacallao et al., 1986).

To summarize, while both analytical and genetic work indicate involvement of proteinaceous material in the process of protein translocation in bacteria, some interpretations of the genetic work, such as coupling between synthesis and translocation or significance attributed to suppressor mutations as revealing involvement of complexes in the translocation process, are questioned. Recently, further $E$. coli genes designated as sec D (Gardel et al., 1987) and sec E (Riggs et al., 1988) were reported to be correlated with protein export in $E$. coli. The localization of products of these genes remains to be elucidated.

\subsection{Organellar Membrane Proteins}

Involvement of proteinaceous material in organellar outer membranes both of mitochondria (Schatz, 1979) and chloroplasts (Chua and Schmidt, 1978, 1979; Highfield and Ellis, 1978) in translocation of migratory proteins has been envisaged. Direct evidence for involvement of proteins in the translocation process came from proteolytic experiments. Mild proteolysis of mitochondria (Argan et al., 1982, 1983; Gasser et al., 1982a) and chloroplasts (Chua and Schmidt, 1978; Cline et al., 1985) prevented post-translational translocation of migratory proteins. Binding studies using unenergized mitochondria or right-side-out vesicles of the outer mitochondrial membrane confirmed that proteinaceous material was required as receptor (Riezman, 1982; Riezman et al., 1983a). Studies on chloroplast envelope vesicles (Pfisterer et al., 1982) led to similar results. In these studies proteolysis prevented binding of precursors to the appropriate membranes.

That different receptors for different categories of migratory proteins of a given organelle might exist was suggested by the observation of Oda et al. (1984) that surface proteolysis of rat liver mitochondria abolished binding of preadrenodoxin, while it did not affect binding of presulfite oxidase. Likewise Zwizinski et al. (1984) reported the differential action of various proteolytic enzymes on receptors for different mitochondrial imported proteins. However, receptors were susceptible to one or the other protease, hence the necessity of using multiple proteolytic enzymes-and the eventual control by other techniques-before concluding a lack of receptor.

Competition studies on inhibition of binding of precursors to organelles favor the presence of receptors. Two types of study were carried out in which 
the inhibitor was constituted by receptors of the outer membranes or by ligands. Thus, Ono and Tuboi (1985) observed that import of the precursor for mitochondrial ornithine aminotransferase was inhibited by the presence of isolated outer membrane vesicles. After detergent solubilization of these vesicles and partial purification and reconstitution of the receptor in lecithin, the liposomes formed also inhibited import into mitochondria. Likewise, Bhat and Avadhani (1985) report that a membrane fraction obtained from mitoplasts inhibited import of carbamylphosphate synthetase by digitonin-treated mitochondria. With regard to inhibition by ligands, it was found that translocation of several mitochondrial proteins into rat liver mitochondria was inhibited by apocytochrome c (Arpin et al., 1980; Matsuura et al., 1981). The nature of proteins, the translocation of which was inhibited, remained undetermined. Recently, however, apocytochrome $\mathrm{c}$ was reported to compete with the uptake of pre-ornithine carbamyl transferase (Hernandez-Yago and Grisolia, 1987). A substantiation in this respect seems useful, since the report is contradictory with another one (see Mori et al., 1985). In Neurospora crassa the import of ADP-ATP carrier, or dicyclohexylcarbodiimide-binding protein (Henning and Neupert, 1981), as well as the insertion of porin into the outer membrane (Zwizinski et al., 1983), is not inhibited by apocytochrome $c$. Thus, different receptor types might be present (Henning and Neupert, 1981). Also, observations by Yaffe and Schatz (1984) with different yeast mutants confirm a differential inhibition of the import of mitochondrial proteins, hence the possible presence of different pathways.

Another set of experiments favoring the possibility of the existence of discriminatory routes for different (categories of) migratory proteins concerned uptake of precursors of these proteins specific to an organ by organelles of another organ. Two proteins of mitochondria of steroidogenic organs, that is, cytochromes P-450 scc (Matocha and Waterman, 1984, 1986; Ogishima et al., 1985 ) and P-450 11 $\beta$ (Matocha and Waterman, 1986) are not imported by heart mitochondria, which, however, can import adrenodoxin, another mitochondrial protein involved in steroidogenesis (Matocha and Waterman, 1986). Likewise, carbamylphosphate synthetase is taken up by mouse liver mitochondria while mitochondria of other tissues (kidney, brain, heart) are unable to import this matrix enzyme (Bhat and Avadhani, 1985). It is worthwhile mentioning at this point that Côté and Boulet (1985) place emphasis only on differences in efficiencies of receptor-translocators of mitochondria of different organs in translocating common or organ-specific proteins.

Besides organ specificity, species specificity was also noted. In numerous cases with mitochondria (Anderson, 1981; Cheng et al., 1987; Horwich et al., 1985a; Schleyer et al., 1982; Schmidt et al., 1983; Takiguchi et al., 1983; Teintze et al., 1982) and chloroplasts (Chua and Schmidt, 1978; Pfisterer et al., 1982), migratory proteins could exchangeably bind to or be taken up and 
processed, depending on the type of experiment carried out, by respective organelles of different species. Also, the firefly (Photinus phyralis) peroxisomal enzyme, luciferase, expressed in the transfected mammalian CV-1 (a monkey kidney) cell line was translocated to peroxisomes (Keller et al., 1987). Likewise expression in $S$. cerevisiae of a peroxisomal enzyme, alcohol oxidase, which this yeast is normally lacking, led to its peroxisomal localization (Distel et al., 1987). However, differences are reported; thus, the precursor of mammalian ornithine carbamyl transferase is not taken up by yeast mitochondria (Takiguchi et al. 1983), and apocytochrome $c$ of $N$. crassa bound to mitochondria from the same origin could not be displaced by apocytochrome $c$ from Paracoccus denitrificans (B. Henning et al., 1983). Also, the precursor of the small subunit of Chlamydomonas reinhardtii ribulose 1,5-bisphosphate carboxylase was not taken up by higher plant chloroplasts (Bedbrook et al., 1980; Coruzzi et al., 1983; Schmidt et al., 1979). In such cases the lack or deficiency of receptors or sequence differences in binding sites of migratory proteins might explain the lack of interspecies uptake.

The import in mitochondria presumably occurs through zones of contact between the outer and the inner mitochondrial membrane. The outer membrane at such zones was recently reported (Dorbani et al., 1987) to have compositional differences from other regions of the outer membrane. Ono and Ito $(1984 a, b)$ found that the receptor for an intermembrane compartment protein resisted the preparation of mitoplasts and was enriched in a part of the outer membrane firmly bound to the inner membrane. Bhat and Avadhani (1985) reported that digitonin-treated mitochondria from mouse liver containing 10 $15 \%$ of outer membrane fragments are able to import $85-90 \%$ of the precursor of carbamylphosphate synthetase taken up by mitochondria. Schleyer and Neupert (1985) and Hartl et al., (1987) have made the interesting observation that in deenergized mitochondria the in vitro synthesized ADP-ATP carrier interacts with the membrane but is not translocated. However, it is kept in an aqueous environment, in a conformation unreactive with proteases (see Figure 5). Upon reenergization of mitochondria, the carrier protein is translocated to the inner membrane, even after trypsinization of mitochondria. Recently, Schwaiger et al., (1987) confirmed that mitoplasts were able to import mitochondrial proteins and that a proteinaceous material was involved in the uptake process. A factor removable by digitonin plus salt from mitoplasts was implicated in the import process. These authors also found that the preformed translocation intermediates remained with mitoplasts after subsequent fractionation. Furthermore, fractionation of mitochondria produced a fraction of intermediate density, as compared to the inner and outer membranes, which was enriched in translocation intermediates. Involvement of contact zones was also assessed by immunocytochemical studies. Recently Soellner et al. (1988) could entrap translocation intermediates of mitochondrial ADP-ATP protein and $F_{1}$-ATPase 
subunit $\beta$ by decreasing the ATP level (or by lowering the temperature) in the in vitro uptake reactions. Such intermediates, which were in the stages of binding to the receptor or inserted in contact sites between the outer and the inner membrane, respectively, could interact with specific antibodies.

Information on the exact nature of the proteinaceous material involved in translocation of migratory proteins is rather scarce. Some observations suggest involvement of intrinsic membrane proteins in mitochondria (Argan et al., 1982, 1983). Recently, Gillepsie (1987) found that a synthetic extrasequence of preornithine carbamyl transferase (residues 1-27) binds specifically in a saturable and reversible manner to a protein of 30,000 Da from the outer membrane of mitochondria as revealed by cross-linking experiments. Mild proteolysis abolished both the binding of the synthetic peptide to and the import of the precursor into mitochondria. The protein that constituted $4-10 \%$ of the outer membrane protein could not be removed by $1 \mathrm{M} \mathrm{KC1}$. Ohba and Schatz (1987) have also recently confirmed the involvement of proteinaceous material in mitochondrial translocation. Treatment of isolated yeast mitochondria with high concentrations of trypsin-inhibited protein import. Also, antibodies to outer membrane proteins or to a specific outer membrane protein of 45,000 Da inhibited the import. However, when mitoplasts were prepared from trypsin-treated mitochondria, import was restored and antibodies to the 45,000-Da protein did not affect the energy-dependent protein import. Thus, involvement of proteinaceous component(s) of the outer membrane and "one or more steps" of translocation would be bypassed when mitoplasts are used. A recent report by Pfanner et al. (1987b) indicated that mitochondrial precursor proteins are imported through a hydrophilic membrane environment. As mentioned above with regard to a similar report concerning translocation of proteins through the microsomal membrane, the observation as such, though highly interesting, does not necessarily prove involvement of membrane proteinaceous material.

With regard to chloroplasts, in order to gain insight into the proteinaceous material interacting with precursor proteins, Kloppstech and Bitsch (1986) labeled in the dark the in vitro-translated precursors of cytoplasmically synthesized pea chloroplast proteins with a heterobifunctional, cleavable, and photoactivatable cross-linker, that is, $\left(\left[{ }^{35} \mathrm{~S}\right]\right.$ cyteamine) $\mathrm{N}$-succinimidyl-3-( (2-nitro4-azidophenyl)-2-aminoethyldithio) propionate (designated as SNAP). Such labeled precursors when presented to isolated chloroplast envelope preparations, labeled envelope proteins of $14,000,24,000,31,000,35,000$, and 42,000 $\mathrm{Da}$. As mentioned above, trypsin treatment of isolated envelopes prevents binding of precursor proteins. Such a treatment of isolated envelopes was shown in the present studies to cleave the 31,000- and 35,000-dalton entities. Recently Pain et al. (1988) confirmed the involvement of a protein of 30,000 daltons by using an antiidiotypic antibody approach which had previously found another application in the field of migratory proteins (see Sakakibara et al., 1987). This 
approach allowed the localization of the 30,000-dalton protein at the contact sites between the two chloroplast membranes. Cornwell and Keegstra (1987), by the use of a heterobifunctional photoactivable cross-linker, were also able to bind the small subunit of ribulose 1,5-bisphosphate to a protein in the outer membrane of chloroplasts. The conjugate was of $86,000 \mathrm{Da}$. This would apparently mean that the membrane receptor protein is of approximately 66,000 $\mathrm{Da}$, provided that a one-to-one stoichiometry of conjugation occurs. However, the efforts of these authors to stain such a protein in the electrophoretograms of the outer membrane remained unsuccessful.

Assays made by Lubben et al. (1987) are remarkable in that they incorporated two different stop transfer sequences of microsomally translocated proteins into precursors for ribulose 1,5-bisphosphate carboxylase; the chimeric precursors were translocated into isolated chloroplasts. The authors conclude that a direct passage through the lipid bilayer is not probable. In support, it can be mentioned that examples exist in which precursors of some hydrophobic proteins (seric apolipoproteins and melitten) cross the membrane and are processed, while they are able to aggregate with or insert into lipid bilayers. It should also be noted that signal sequences were even observed to cross the microsomal membranes when inserted in some chimeric constructs (see Section 4.1).

Several reports (Argan et al., 1983; Miura et al., 1983; Otha and Schatz, 1984; Takiguchi et al., 1983) also indicate the presence of cytosolic factor(s) that are proteinaceous in nature (Argan et al., 1983; Takiguchi et al., 1983) and involved in the translocation process. A low-molecular-weight material of less than $5000 \mathrm{Da}$ (Argan et al., 1983), which is dialyzable and destructible by trypsin (Miura et al., 1983), as well as a 40,000-Da protein (Otha and Schatz, 1984) and ions $\left[\mathrm{Mg}^{2+}\right.$ (Lewin and Norman, 1983), $\mathrm{K}^{+}$(Miura et al., 1983)] were involved in mitochondrial transport. Implication of a nucleoprotein complex of $M_{\mathrm{r}} 400,000$ in this process was also mentioned (Firguira et al., 1984) and the presence of "import factor(s)" in reticulocyte lysates was reported. This factor(s) would bind the precursor forming a 5S complex. However, the factor(s) alone does not bind to mitochondria (Argan and Shore, 1985) and, importantly, RNA is not required for mitochondrial import (Argan and Shore, 1985; Burns and Lewin, 1986). Recently Joste et al. (1987) confirmed the presence of a stimulating "import factor' for three mitochondrial proteins in reticulocye lysates and Ono and Tuboi (1988) proposed that precursors of mitochondrial proteins would bind to and form a complex with the "import factor' 'which is recognized by the membrane receptor. Behra and Christen (1986) have presented evidence that both in vivo and in vitro the precursor of aspartate aminotransferase forms high-molecular-weight complexes presumably involved in translocation. It would thus seem clear that a simple interaction of precursors and lipids is far from representing the reality of migratory protein translocation. 


\section{ENERGY DEPENDENCE OF PROTEIN TRANSLOCATION PROCESSES}

\subsection{Secretory and Transmembrane Integral Proteins}

Protein translocation through the microsomal membrane was thought not to require energy in addition to the amount used for chain translation. Since the process occurred cotranslationally, it appeared unpractical to experiment for the requirement for additional energy. Often, when under some experimental conditions proteolytic processing of exported proteins in prokaryotic systems did not occur, the lack of cleavage of the extrasequence was taken as revealing the lack of translocation, and conclusions as to energy requirement for the last process were drawn. It should, however, be borne in mind that for many secretory and transmembrane integral proteins a cleaved signal sequence does not exist. Furthermore, under various experimental circumstances, processing and translocation could be experimentally dissociated, so that processing did not occur or was delayed while translocation had occurred (DiRienzo and Inouye, 1979; Freudl et al., 1985; Giam et al., 1984; Halegoua and Inouye, 1979; Halegoua et al., 1976, 1977; Ichihara et al., 1982; Inouye et al., 1983a,b; Koshland et al., 1982; Lin et al., 1978, 1980; Lory et al., 1983; Russel and Model, 1981; Seehara and Khorana, 1984; Sekizawa et al., 1977; Wu et al., 1977).

Studies carried out on $E$. coli-exported proteins indicated that, even under normal conditions, while some of them were cotranslationally processed, others were processed co- and post-translationally and still others post-translationally (Josefsson and Randall, 1981a,b; Tweten and Iandolo, 1983). The observation that some $E$. coli exported proteins could be cleaved by an externally added proteinase $\mathrm{K}$, only after translation of $80 \%$ (maltose-binding protein) or the full length (ribose-binding protein) of the chain, was interpreted to mean that a strict coupling between the translation and translocation does not exist (Randall, 1983). While such an interpretation is attractive, the alternative, that the translocated chains were not accessible to proteinase $\mathrm{K}$, in the context of the membrane, cannot be excluded by such observations alone. In other words, problems of crypticity should be taken into account (see Etémadi, 1980a, 1985).

The possibility of translocation of exported proteins without the cleavage of signal sequence rendered equivocal the interpretation in terms of the lack of translocation of secretory and transmembrane proteins of bacteria that remained unprocessed as a result of treatment of cells with various drugs. In several cases uncouplers were observed to prevent processing (Daniels et al. 1981; Date et al., 1980; Enequist et al., 1981; Palva et al., 1981; Russel and Model, 1981; Tweten and Iandolo, 1983; Valusek et al., 1984). However, these drugs did not prevent translocation in all cases (Ghrayeb and Inouye, 1984; Russel and Model, 1981; Valusek et al., 1984; Zimmermann et al., 1982) and, in addition, 
the reported effect of uncouplers on the action of signal peptidase (Russel and Model, 1981; Zimmermann et al., 1982) rendered the knowledge of the exact cellular location of precursors in the presence of these drugs mandatory. In fact, in early works, the cytoplasmic localization was assessed only in some cases (Date et al., 1980; Ghrayeb and Inouye, 1984; Wolfe and Wickner, 1984; Zimmermann and Wickner, 1983) and a (slow) translocation after removal of uncouplers was shown (Ghrayeb and Inouye, 1984). The last experiment, translocation of the cytoplasmically located precursor after removal of the drug is in fact required for the demonstration of a post-translational energy-dependent translocation, since an effect of drugs on the membrane conformation preventing translocation cannot be excluded, as also pointed out by others (Muren and Randall, 1985). Despite these notes of caution, other in vitro experiments (Chen and Tai, 1985, 1987a; Mueller and Blobel, 1984) have shown the feasibility of the post-translational energy-dependent translocation of exported proteins in prokaryotic systems. With regard to eukaryotic systems, it was observed by different laboratories (Hansen et al., 1986; Rothblatt and Meyer, 1986b; Waters and Blobel, 1986) that yeast pre-pro- $\alpha$-factor synthesized in vitro can be taken up post-translationally in an ATP-dependent manner into yeast microsomes. However, such an uptake was limited to the mentioned case and, for example, it did not work for invertase, another yeast secretory enzyme. A report by Maher and Singer (1986), stating that the precursor of a protein, the translocation of which was previously found to be co-translational, could occur posttranslationally after dithiothreitol treatment would have constituted a clear-cut invaluable demonstration of the feasibility of post-translational microsomal uptake of proteins. Ibrahimi (1987), however, reports that the precursor in this case was still linked to ribosomes.

As to the nature of energy involved in translocation in prokaryotic cells, the involvement of membrane potential was first suggested by Date et al., (1980). Bakker and Randall (1984) did not admit this proposal and suggested instead the involvement of both the $\mathrm{pH}$ gradient $(\Delta \mathrm{pH})$ and membrane potential $(\Delta \psi)$ components of the proton motive force, while whether the effect is at the level of translocation or only processing was not decided. Mueller and Blobel (1984) found that the presence of a functional $\mathrm{F}_{0}-\mathrm{F}_{1}$ ATPase, hence a proton motive force, was absolutely necessary for protein translocation in $E$. coli. Enequist $e t$ al., (1981) had previously correlated processing of exportable proteins in $E$. coli preferentially with the maintenance of the proton motive force, but a direct involvement of ATP had not been excluded. There are observations on bacteria, Streptomyces fecalis and strains of $E$. coli revealing that proton motive force is not required for growth (Harold and Van Brunt, 1977; Kinoshita et al., 1984), hence for synthesis and insertion of membrane components, in these cases. Chen and Tai (1985), having carried out studies on inside-out inner membrane vesicles, insist on the essentiality of ATP as an energy source, while a proton motive force would maintain the membrane topology only for a more 
efficient translocation. In recent work these authors (Chen and Tai, 1987a) observed that membrane perturbants such as ethanol, procaine, and phenethyl alcohol at low concentrations inhibit ATP-dependent protein translocation through $E$. coli inner membrane vesicles. Also, the optimal translocation of pre-OmpA in such a system was confirmed to require both the membrane potential and ATP (Geller et al., 1986) and Yamane et al., (1987) have reported that translocation on an OmpF-lipoprotein chimeric protein through inverted $E$. coli inner membrane vesicles involved both components of proton motive force and ATP. ATP had to be generated in the experiment medium; addition of ATP itself did not work. Other authors (Chen and Tai, 1987b) confirm the requirement for an ATP-generating system. They also present evidence indicating requirement for ATP under conditions of cotranslational protein translocation through $E$. coli-inverted inner membrane vesicles.

With regard to protein translocation in eukaryotic cells, in vivo experiments on yeast indicated that DNP inhibited a thorough translocation of the secretory protein invertase (Ferro-Novick et al., 1984a,b). Since uncouplers were reported not to inhibit protein translocation at the level of microsomal membrane (Hansen et al., 1986; Rothblatt and Meyer, 1986b; Waters and Blobel, 1986, see, however, Rees-Jones and Alawqati, 1984), it is possible to imagine an indirect in vivo effect of DNP at the level of mitochondrial ATP synthesis; a subsequent decrease of ATP level in the cytoplasm would be responsible for the defect in translocation. In line with such a possible explanation is the observation that in vitro post-translational protein translocation in microsomes under particular experimental conditions required the presence of GTP, creatine phosphate, and creatine phosphokinase (Perara et al., 1986). It is also reported that cleavage of a phosphodiester is required for the post-translational insertion of the $\mathrm{N}$-terminal segment of the glucose transporter comprising 8 out of 12 membrane-spanning domains of the molecule (Mueckler and Lodish, 1986).

\subsection{Migratory Proteins}

Since the early observations mentioned above, numerous authors have confirmed the energy requirement for protein translocation in mitochondria (e.g., see Anderson, 1981; Chien and Freeman, 1983; Fenton et al., 1984; Gasser et al., 1982a; Ikeda et al., 1987; Kolansky et al., 1982; Lewin and Norman, 1983; Maccecchini et al., 1979a,b; Mori et al., 1981, 1985; Morita et al., 1982; Ono and Ito, 1984b; Ono et al., 1985; Schleyer et al., 1982; Sidhu and Beattie, 1983; Teintze et al., 1982; Wandinger-Ness and Weiss, 1987; White and Sandalios, 1987; Zimmermann and Neupert, 1981; Zwizinski and Neupert, 1983; Zwizinski et al., 1984) and chloroplasts (e.g., see Cline, 1986; Cline et al., 1985; Coruzzi et al., 1983; Fluegg and Hinz, 1986; Grossman et al., 1980; Pfisterer et al., 1982). 
As to the step at which the energy is necessary in the process of protein translocation, it had been previously envisaged that either the processing or the translocation step could be involved. The suggestion that the post-translational protein translocation can be assimilated to "some sort of active transport" (Etémadi, 1980a) favored the essential implication of energy at the level of translocation, processing being a consequence of translocation. That the step of translocation, not that of cleavage, is energy requiring was confirmed by the observation of Zwizinski and Neupert (1983) that in the presence of $o$-phenanthroline, an inhibitor of mitochondrial processing enzyme, imported proteins were translocated without processing. Similar observations were made with bovine adrenal cortex mitochondria with regard to the cleavage of precytochrome P-450 scc and precytochrome P-450 11 $\beta$ (Ogishima et al., 1985; Ou et al., 1986). Also, import of cytochrome P-450 scc by bovine adrenal cortex mitochondria could occur at relatively low temperature at which processing by the cleavage enzyme was inhibited (Ou et al., 1986). That processing is not an absolute requirement for translocation of migratory proteins could be confirmed during observations made on hybrid proteins bearing the extension peptide of the matrix enzyme ornithine carbamyl transferase plus 5 or $28 \mathrm{~N}$ terminal amino acids of the matrix enzyme and the full length of $E$. coli asparagine synthetase, a soluble protein. In vitro translocation experiments indicated that only the second hybrid was correctly processed. Presumably, the mature part contrib-

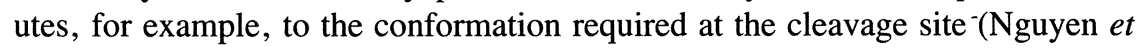
al., 1986). Further work by Nguyen et al. (1987) has shown that when a deletion in residues 22-30 of the presequence of the enzyme was introduced, the mutant precursor could still be taken up into the matrix of isolated mitochondria while cleavage of the shortened signal did not occur despite the presence of the cleavage site (between Gly 32 and Ser 33). Vassarotti et al. (1987a) observed that small deletions distal to the cleavage site of the precursor of $\beta$-subunit of $\mathrm{F}_{1}$-ATPase prevented maturation but that immature chains were, however, assembled in a functionally active enzyme, indicating that import and maturation have distinct requirements. The transit sequence of the precursor of the algal (Chlamydomonas reinhardtii) small subunit of ribulose 1,5-bisphosphate carboxylase is required for translocation through the envelope of vascular plant (pea, spinach) chloroplasts. However, cleavage to the mature size does not occur; only an intermediate is formed. It was suggested that processing of the precursor occurs in two steps, one of which is conserved during evolution (Mishkind et al., 1985).

It is thus apparent that the energy-dependent translocation of mitochondrial and chloroplast migratory proteins and their processing have essentially distinct requirements, those of cleavage being principally governed by the activity of the cleavage enzyme in which the conformation of the cleavage site plays an important role (see also Miura et al., 1986). A remarkable observation (Teintze et al., 1982; Zwizinski et al., 1984) is that in unenergized mitochondria, the 
binding of some mitochondrial proteins is significantly reduced or does not occur. Thus, an appropriate membrane conformation, presumably exposing the binding sites for some migratory proteins, is influenced by membrane energization.

As to the form under which the energy is used, it was first admitted that ATP as such is required (Nelson et al., 1979). However, further studies led to the conclusion that the membrane electrical potential was involved (Pfanner and Neupert, 1985; Schleyer et al., 1982) or that the proton motive force would intervene, although it was not decided if $\Delta \mathrm{pH}$ or $\Delta \psi$ was used (Gasser et al., 1982a). More recently, import of subunit $\beta$ of the mitochondrial $F_{1}$-ATPase was found to depend on both the membrane potential and ATP (Pfanner and Neupert, 1986). Other authors have found that ATP, in addition to an energized inner membrane, was required for import of proteins into mitochondria. GTP could replace ATP. In this action ATP exerted its effect from outside the mitochondria (Eilers et al., 1987). Nucleotide triphosphates were suggested to act on precursors of imported proteins (Chen and Douglas, 1987a,b; Pfanner et al., 1987a; Verner and Schatz, 1987) in unfolding them (Pfanner et al., 1987a, 1988 ) in a process involving the cleavage of a phosphodiester bond (Chen and Douglas, 1987a,b) conversely, however, Eilers et al. (1988) more recently proposed a requirement for membrane potential for unfolding a precursor at the surface of the membrane, while ATP would be required for import and not the membrane potential.

Studies on import of proteins to chloroplasts led to the proposal that the presence of ATP in the stroma compartment is required (Grossman et al., 1980; Pain and Blobel, 1987). However, Fluegg and Hinz (1986) report on the requirement for ATP from outside the envelope and suggest the involvement of a phosphorylation-dephosphorylation cycle. The reason(s) for the discrepancy is not clear. It would appear that the supply of ATP in the stroma compartment would be required for protein insertion into the thylakoid membrane and for uptake of intrathylakoid luminal proteins, since there are indications that proteins of thylakoid membrane (such as light-harvesting chlorophyll a/b proteins) and presumably those of the intrathylakoid compartment are first imported and released in the stroma, then taken up by thylakoid membranes in a process requiring ATP and soluble factor(s) from stroma (Cline, 1986). (For more information, see Section 4.2.2.)

The only mitochondrial migratory protein for which the translocation does not require an energized membrane is apocytochrome $c$ (Henning and Neupert, 1981; Nelson et al., 1979; Zimmermann and Neupert, 1981). However, what is released in the intermembrane space is cytochrome $c$ itself (Henning and Neupert, 1981). Thus, as previously discussed (Etémadi, 1985), a simple diffusion of apocytochrome $c$ does not explain translocation, the case being reminiscent of group translocation processes. Considering, in addition, the fact that 
"proteins" of the outer membrane were found to bind the apocytochrome $c$ (Zwizinski et al., 1984), I have also suggested that the completion of translocation itself can involve the enzyme "apocytochrome $c$ heme lyase." Recently, different authors appreciated the exact localization of the enzyme. Enosawa and Ohashi (1986) locate it on the outer surface of the inner mitochondrial membrane, presumably in contact zones between the outer and the inner membrane, while Nicholson et al., (1987) find it in the intermembrane space. However, the last authors confirm that the enzyme, which is found not to exist in a stable complex with the binding protein, "plays a central role in transfer of apocytochrome $c . "$ As also suggested previously (Etémadi, 1985), it would appear useful to keep in mind this interesting example; maybe other specific examples of group translocation processes could be encountered in protein translocation processes, despite the fact that processing reactions are generally not coupled with or do not furnish the driving force for the act of translocation and can be dissociated from it.

Contradictory indications exist regarding the energy requirement for protein translocation to microbodies. Uptake of the precursor of glyoxysomal malate dehydrogenase from watermelon (Citrullus vulgaris) by glyoxysomes of castor bean ( $R$. communis) was reported not to be energy requiring (Gietl and Hock, 1986). However, Goodman (1985) suggested the requirement for an energized state of peroxisomes, possibly through the presence of a proton pump, for translocation of peroxisomal enzyme, alcohol oxidase, in Candida boidinii and, recently, Douma et al. (1987) have reported the presence in purified peroxisomes from Hansenula polymorpha of a $\mathrm{H}^{+}$-ATPase resembling the mitochondrial enzyme.

\section{TRANSLOCATION AND MEMBRANE-ANCHORING SIGNALS}

\subsection{Signal and Membrane-Anchoring Sequences for Proteins Translocated through the Endoplasmic Reticulum and the Bacterial Cytoplasmic Membrane}

\subsubsection{Structural Features of Signal Sequences}

Concentrating on active transport, the general mechanism governing cellular protein translocation processes, imposes the involvement of membrane proteinaceous components interacting with precursors on the one hand and energy consumption on the other. A detailed mechanistic knowledge of the process of translocation, in the framework of the active transport, should then be sought. The most fruitful part in the signal hypothesis (Blobel and Dobberstein, 1975; Milstein et al., 1972) has been the proposal of the involvement of signals at the $\mathrm{N}$ termini of exported proteins in triggering their translocation, despite 
the fact that early proposals on the community of (Blobel and Sabatini, 1971) or a high homology between (Devillers-Thiery et al., 1975) signal sequences of different exported proteins was soon observed to be unverified.

Several secretory and transmembrane integral proteins are devoid of cleaved signal sequences as first shown for ovalbumin (Palmiter et al., 1978), then observed in numerous other cases especially of membrane proteins (Bonatti and Blobel, 1979; Chiocchia and Dirckamer, 1984; Chyn et al., 1979; Fields et al., 1981; Ghersa et al., 1986; Gorin et al., 1984; Holland et al., 1984; Kopito and Lodish, 1985; Mc Lennan et al., 1985; Mueckler et al., 1985; Noda et al., 1984; Paul and Goodenough, 1983; Schechter et al., 1979; Spiess and Lodish, 1985; Spiess et al., 1985; Strubin et al., 1984; Zerial et al., 1987), including several microsomal transmembrane integral proteins (Armstrong et al., 1984; Bar-Nun et al., 1980; Black et al., 1979; Brown and Simoni, 1984; Gonzalez and Kasper, 1980; Heinemann and Ozols, 1984; Kasper and Porter, 1985; Lauffer et al., 1985; Okada et al., 1982) and bacterial inner (Brusilow et al., 1981; Büchel et al., 1980; Ehring et al., 1980; Higgins et al., 1982; Innis et al., 1984; Poulis et al., 1981; Santos et al., 1982; Wolfe et al., 1983; Yazgu et al., 1984) and some outer (De Geus et al., 1984) membrane proteins. In such cases uncleaved signals are either located $\mathrm{N}$ terminally or, in some cases, internal to the chain.

Cleaved sequences can be of 15-36 amino acid residues (see Abrahamsen et al., 1985; Watson, 1984). Inherent in the loop model (DiRienzo et al., 1978; Inouye and Halegoua, 1980) is the recognition of three regions in signal sequences, an $\mathrm{N}$-terminal segment comprising positively charged residues, followed by a hydrophobic segment divided by a proline or a glycine residue, then the region comprising the cleavage site at the $\mathrm{C}$ terminus. Table I shows signal sequences of some exported proteins of eukaryotic and prokaryotic cells. The $\mathrm{N}$ terminus ( $\mathrm{n}$ region) is of variable length and composition comprising one or two positively charged residues in eukaryotic and prokaryotic sequences, respectively (Von Heijne, 1985). Often, signal sequences of gram-positive bacteria are "unusually" long - that of the staphylococcal protein A being 36 residues; they also have on average more than 3 positively charged amino acid residues at their $\mathrm{N}$ termini (Abrahamsen et al., 1985; Watson, 1984). The minimal length of the hydrophobic segment (h-region) is of some 8 residues and the $\mathrm{c}$ region ( $\mathrm{C}$ terminus) comprising the cleavage site, is formed of 5 or 6 residues in eukaryotic and prokaryotic signal sequences, respectively (Von Heijne, 1985). The C-terminal segment, comprising the cleavage site, was proposed to follow the " $-3,-1$ "' rule. The importance of the conformation of the polypeptide chain near the cleavage site of the prepeptide for the action of the signal peptidase, in particular the frequency of the breakage of repeating conformations, and the fact that the cleavage occurs, in general, at the $\mathrm{C}$ terminus of an uncharged or small amino acid residue were previously noted (Austen, 1979). The " $-3,-1$ " rule defines the amino acid residues most frequently encountered 


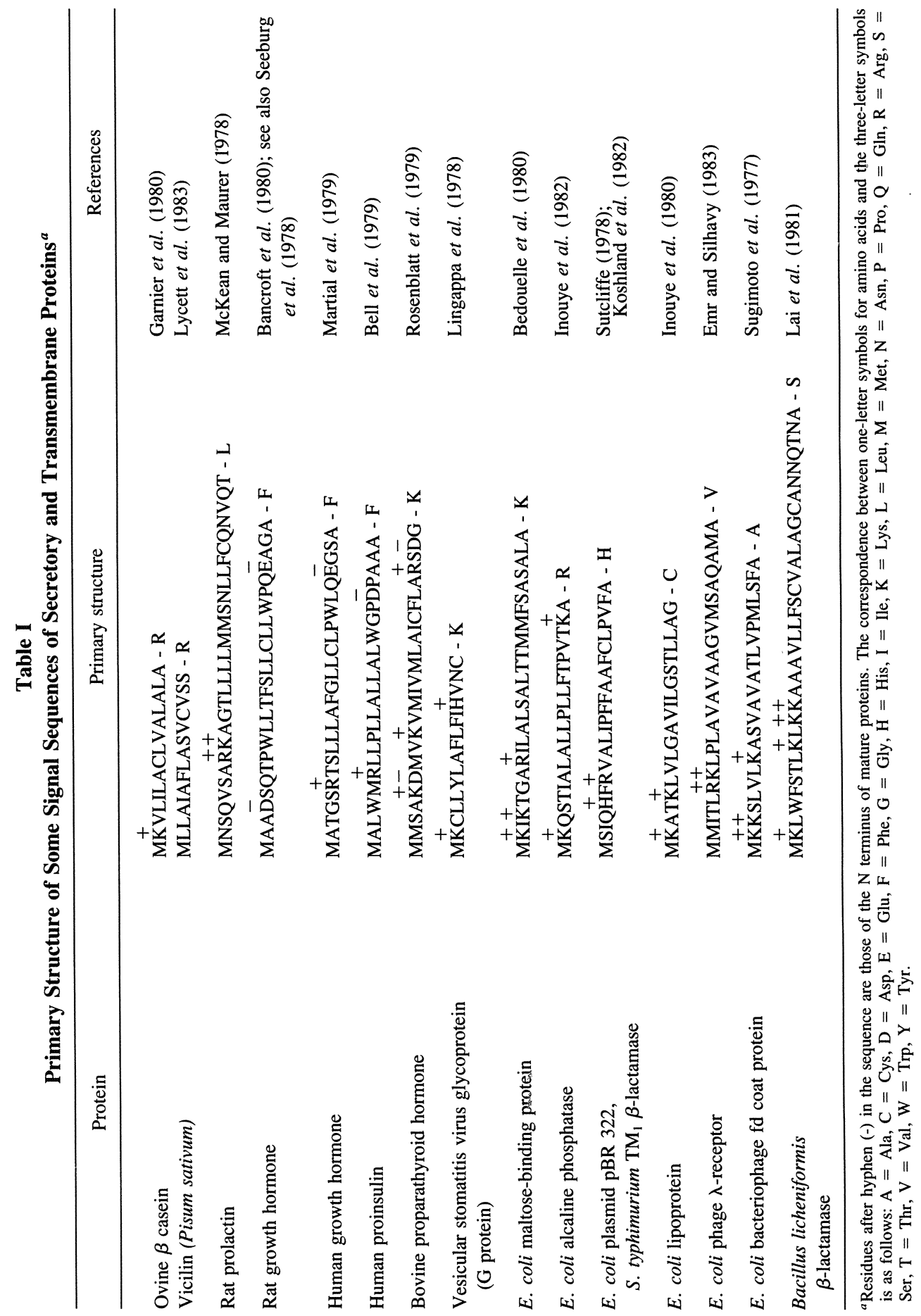


around the cleavage site (Perlman and Halvorson, 1983; Von Heijne, 1984). However, this rule suffers several exceptions (see references in Ibrahimi and Gentz, 1987).

A question of importance with regard to the detailed molecular events governing protein translocation concerns the part of the signal sequence entering in interaction. Some observations were interpreted as favoring the involvement of charged residues at the $\mathrm{N}$ terminus of the signal sequence in the export of $E$. coli lipoprotein (Inouye et al., 1982; Inukai and Inouye, 1983). Other studies indicate that no stringent conditions as to the conformation of the proximal segment of signal sequences should exist (Brown et al., 1984). However, there are data indicating that the presence of positively charged residues in the $\mathrm{n}$ region is dispensable for correct translocation (Kaiser et al., 1987; Lipp and Dobberstein, 1986b). For a fusion precursor bearing the signal of lipoprotein and 9 amino acids of the $\mathrm{N}$ terminus of its mature chain plus $\beta$-lactamase sequence deleted of its own signal, it could be observed by Garcia et al., (1987) that translocation through the mammalian microsomal membrane could occur. Mutations that suppressed positively charged residues did not affect translocation and processing, which, however, occurred at a potential cleavage site for signal peptidase I but not for signal peptidase II, which is absent in microsomes. These studies also showed that interaction with SRP was not affected as a result of substitutions in the positively charged residues. Of course, in this and other cases, involvement of initiating $\mathrm{N}$-terminal methionine in eukaryotic systems cannot be excluded. With this reservation in mind, however, the lack of involvement of positively charged amino acid residues is also supported by the presence of rare translocated proteins such as vicilins (Lycett $e t$ al., 1983) the signal sequences of which lack positively charged residues at their $\mathrm{N}$ termini or even the presence of signal sequences such as that of rat growth hormone (Bancroft et al., 1980; Seeburg et al., 1978) with a negatively charged residue in the $\mathrm{N}$-terminal region (see Table I). Thus the requirement for positively charged residues within the signal sequence is not substantiated. This does not mean an insensitivity of the translocation process to manipulations in the charge of the signal. In a recent study, Freudl et al. (1988) have shown that depending on the nature of mutation introduced in the region coding the signal sequence of the OmpA-protein gene, this $E$. coli protein can either be translocated entirely post-translationally or entirely co-translationally: when a positive charge was added near the $\mathrm{N}$-terminal and the hydrophobic region was shortened, the translation became post-translational; when the hydrophobic region was lengthened, the translocation was co-translational. During studies on interchangeability between signal sequences of translocated proteins of bacterial and eucaryotic cells (Tallmadge et al., 1980a,b, 1981), as well as during other experiments (Perara and Lingappa, 1986; Rothstein et al., 1984; Simon et al., 1987; Wiedmann et al., 1986b), it appeared that signal sequences ex- 
tended at their $\mathrm{N}$ termini and thus made internal are still operative. For example, when a plasmid was constructed in such a way that it could code for preprolactin and an additional 250 amino acids (part of two globin chains in tandem) localized $\mathrm{N}$ terminally to the precursor chain, it was observed that not only prolactin but also its signal peptide together with the long $\mathrm{N}$-terminally added sequence was translocated through the microsomal membrane in vitro and through the endoplasmic reticulum of Xenopus laevis oocytes during in vivo experiments, although in the last case the translocated extended signal sequence was mostly degraded (Simon et al., 1987). A remarkable case is the one reported by Wiedmann et al. (1986b) in which the extension segment added $\mathrm{N}$ terminally was highly charged; it did not remain cytoplasmic, however, and was translocated to the side opposite to the cytoplasm, revealing again that either an electrostatic interaction with the membrane did not occur or, if it did occur, the interaction did not result in the cytoplasmic location of the $\mathrm{N}$ terminus as would have been expected from different models (see Figure 1).

Several studies concerned the central hydrophobic segment of signal sequences. Substitution (e.g., that of hydroxyleucine for leucine) (Gordon et al., 1981; Hortin and Boime, 1980a,b) in the signal peptide of eukaryotic secretory proteins and mutational modifications on the signal sequence of $E$. coli-exported proteins (Bedouelle et al., 1980; Emr and Silhavy, 1982, 1983; Emr et al., 1980; Michaelis et al., 1983a,b) confirmed the requirement for the hydrophobicity of this region which had to be able to exhibit the $\alpha$-helical conformation (Emr and Silhavy 1983; Fikes and Silhavy, 1987). Kendall et al. (1986), using site-directed mutagenesis, generated various presequences in the precursor of $E$. coli alkaline phosphatase (see Table I); such modifications pointed again to the importance of a structural feature of the hydrophobic segment rather than the primary sequence. Interestingly, Ryan et al. (1986) found that the presence of a charged residue introduced in the hydrophobic region by mutational substitution does not necessarily alter the function of the signal sequence provided that by substitution of other residues the hydrophobicity level of the segment was retained. Other authors (Blachly-Dyson and Stevens, 1987) also report that introduction of an aspartate residue in the hydrophobic core of the signal sequence of yeast vacuolar enzyme, carboxypeptidase $\mathrm{Y}$, does not affect its translocation. Furthermore, a recent remarkable report by Kaiser $e t$ al. (1987) indicates that, in the case of yeast invertase, many random sequences with no more than 2 or 3 contiguous hydrophobic amino acid residues can still function efficiently in translocation of the protein in yeast. Thus, a contiguous stretch of 7 or 8 hydrophobic residues is not an absolute requirement. Likewise, Smith et al. (1987) by "shotgun cloning", of restriction fragments of $B$. subtilis chromosomal DNA obtained a high frequency of "export signal coding regions." Such signals placed $5^{\prime}$ to truncated genes, deleted from the signal sequence coding region of exported proteins, could direct transloca- 
tion of respective proteins both in $B$. subtilis and $E$. coli harboring plasmid vectors. More striking is the observation made by Blachly-Dyson and Stevens (1987) that deletion of signal sequence of yeast vacuolar carboxypeptidase $\mathrm{Y}$ does not totally abolish its post-translational translocation and glycosylation. Among other possibilities the eventuality of the presence of an internal, but less efficient, signal was envisaged, although a typical signal could not be detected in the sequence. Finally, an important point should be emphasized: not only can translocated proteins have signals that naturally or under particular experimental circumstances remain uncleaved, but also the signal and the cleaved sequences are not always synonymous, since the cleaved sequences can have dispensable amino acid residues or fragments, or the significant part of the signal might not correspond to the cleaved segment. Thus, the $\mathrm{N}$-terminal cleaved sequence of the 13 amino acids (see Seehara and Khorana, 1984) of bacteriorhodopsin presumably does not constitute (see Etémadi, 1986a) all of the translocation signal. Storm and Lory (1987) have recently reported that the cleaved sequence of pilin in Pseudomonas aeruginosa is formed only of $6 \mathrm{~N}$-terminal amino acid residues. Translocation involves an additional sequence from the $\mathrm{N}$ terminus of the mature protein. Also, a regulatory role for the signal sequence, influencing (diminishing) the rate of synthesis of exported proteins through its interaction with the translation system, was recently reported by Ibrahimi and Gentz (1987).

\subsubsection{Structural Features of Membrane-Anchoring Sequences of Transmembrane Integral Proteins}

When truncated precursors of transmembrane proteins were expressed, if the deletion at the $\mathrm{C}$ terminus comprised the anchoring domain, proteins were secreted, confirming that early events of secretion and membrane insertion are similar (Bremer et al., 1982; Boeke and Model, 1982; Gething and Sambrook, 1982; U. Henning et al., 1983; Rose and Bergman, 1982; Sveda et al., 1982). Conversely, when modified secretory proteins to which a membrane-anchoring domain of a transmembrane protein was added were expressed, the hybrid protein remained membrane inserted (Boeke and Model, 1982; Guan and Rose, 1984; Rizzolo et al., 1985).

Transmembrane integral proteins often contain, at the cytoplasmic side, positively charged residues close to the membrane-anchored hydrophobic domain. Such residues were found to be dispensable with regard to membrane anchorage of these proteins (Davis and Model, 1985; Davis et al., 1985). Other studies indicated that the hydrophobic domain can be shortened to 12 amino acid residues (instead of 20) in the G protein of the vesicular stomatitis virus or that a charged residue can be inserted in the hydrophobic domain with conservation of the membrane topology, if a stretch of 11 uninterrupted hydrophobic residues was maintained (Adams and Rose, 1985a,b). Culter and Garoff 
$(1986 a, b)$ have found that, as a result of replacement of the cytoplasmic positively charged residues by neutral or negatively charged residues or substitution of a hydrophobic residue by an acidic residue in the hydrophobic core of the transmembrane segment, although the location of the protein is not affected, its stability in the membrane is diminished. Recently, in contrast with the above observations, experiments interpreted as revealing the requirement for the cytoplasmic carboxyl termini of some $E$. coli membrane proteins for their insertion in the membrane were reported (Dalbey and Wickner, 1986; Kuhn et al., 1986). Unfortunately, the lack of insertion was not demonstrated but only conjectured because of inaccessibility to protease action from outside; that is, the notion of crypticity was overlooked. Remarkable is the observation by Watanabe et al. (1986) that the precursor of an E. coli exported protein could be incorporated in $E$. coli inner membrane, while it passed through the microsomal membrane and become luminal. However, since in the second case the protein is glycosylated (once or twice) in its polypeptide chain, it would appear interesting to learn what the location of the protein would be if glycosylation were inhibited by tunicamycin, since it cannot be excluded that glycosylation in this particular case might play a role on the protein location.

\subsubsection{Can a Signal Sequence Act as a Membrane-Anchoring Sequence and Vice Versa?}

In some cases, the possibility for a signal sequence to act as an anchoring sequence could be observed (Coleman et al., 1985) or suspected (Seeburg et al., 1978), while other authors insist on a functional difference between these two types of sequence (Yost et al., 1983). Finidori et al. (1987) have recently observed that a signal sequence, that of the influenza virus hemagglutinin, when made internal in a chain, was neither cleaved nor led to membrane anchoring but was translocated. In contrast, the $\mathrm{N}$ terminus of cytochrome P-450, which normally anchors this cytochrome to the microsomal membrane with a cytoplasmic location of the $\mathrm{C}$ terminus of the protein, could anchor a chimeric protein in the microsomal membrane. In both these cases a genuine signal sequence at the $\mathrm{N}$ terminus of chimeric constructs initiated the translocation process. Tabe et al. (1984), however, report that the uncleaved signal sequence of ovalbumin can act as an anchoring sequence. In a different instance of membrane proteins with a cytoplasmically located $\mathrm{N}$ terminus and an extracytoplasmic $\mathrm{C}$ terminus, the signal sequence and the anchoring sequence may be the same segment of the protein, as suggested by Claesson et al. (1983) and Holland et al. (1984) (see also Bos et al., 1984; Spiess and Lodish, 1986). Zerial et al. (1986) have shown that the hydrophobic membrane-spanning domain of such a protein, human transferrin receptor, was necessary and sufficient (in the case of hybrid constructs) for translocation and insertion, that is, for acting as both signal and membrane-anchoring sequence. Spiess and Hand- 
schin (1987) have likewise shown that for asialoglycoprotein receptor $\mathrm{H}_{1}$, a hydrophobic stretch of 10-12 residues was essential for membrane insertion. A report by Lipp and Dobberstein (1986b) concerning another example of these proteins is remarkable. The authors worked on hybrids made of the $\mathrm{N}$ terminus of the human invariant $\gamma$ chain of class II histocompatibility antigens and a soluble cytoplasmic protein, chloramphenicol acetyltransferase (CAT); the invariant $\gamma(\mathrm{I} \gamma)$ has a short $\mathrm{N}$-terminal segment exposed cytoplasmically, while the bulk of the protein is extracytoplasmic. When the hybrid (I $\gamma$-CAT) was such that most of the exoplasmic domain of I $\gamma$ was replaced by CAT chain, it became inserted in the membrane (Figure 3A). The CAT chain became exoplasmic and glycosylated. When the cytoplasmic domain of 30 amino acids comprising positively charged residues was deleted, so that the hybrid comprised only the hydrophobic membrane-spanning segment (also approximately of 30 residues) of the invariant chain plus 9 residues of the $\mathrm{N}$ terminus of the mature chain and the CAT sequence, the enzyme was processed and secreted.
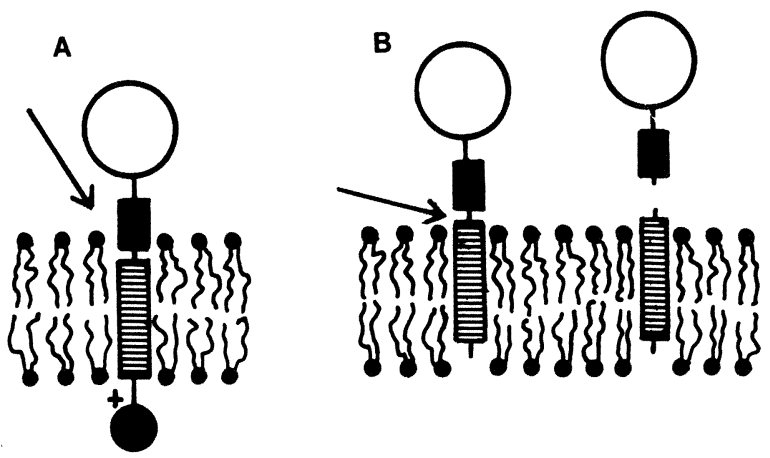

FIGURE 3. This figure (see Lipp and Dobberstein, 1986b) shows how the hydrophilic cytoplasmic domain of a putative uncleaved signal sequence might prevent access of its cleavage site to the cleavage enzyme by causing its burying in the membrane. In (A) a hybrid protein in which the $\mathrm{N}$-terminal segment of the I $\gamma$ chain of the class II histocompatibility antigens-which normally exposes its $\mathrm{N}$-terminal cytoplasmically and its $\mathrm{C}$-terminal extracytoplasmically-is linked to a cytoplasmic soluble protein, chloramphenicol acetyltransferase. The plain disc and most of the dark rectangle show the soluble enzyme. The other part of the dark rectangle, the striated rectangle (the hydrophobic membrane embedded domain) and the dark disc (the short cytoplasmic domain) represent the $\mathrm{N}$ terminus of $\mathrm{I} \gamma$ chain. The putative cleavage site is shown by an arrow and charges in the cytoplasmic domain close to the hydrophobic segment by + . Such a hybrid is inserted into the membrane with the orientation shown by $\mathrm{I} \gamma$ itself, that is, $\mathrm{N}$ terminus in the cytoplasmic side. The charged residues would prevent the displacement of the hybrid in the direction perpendicular to the membrane surface. As a consqeuence, cleavage, presumably by signal peptidase, cannot occur. In (B) the cytoplasmic $\mathrm{N}$ terminus, including charged residues of $\mathrm{I} \gamma$, is omitted. Consequently, the putative cleavage site can reach the extracytoplasmic side in such a way that cleavage can now occur. 
The interesting possibility was suggested that normally the cleavage site of the signal sequence, despite its presence, cannot reach the active site of the signal peptidase. Removal of the cytoplasmic segment comprising charged residues would allow the mobility of the membrane-inserted segment in the direction perpendicular to the plan of the membrane so that the active site of the cleavage enzyme can be reached. In another interesting report Zerial et al. (1987) indicate that replacement of the anchoring domain of human transferrin receptor by membrane-anchoring domains of either Semliki forest virus P 62 protein or hemagglutinin as well as a completely artificial uncharged peptide led to translocation and membrane anchoring, thus confirming that membrane-spanning domains of transmembrane proteins can also act as translocation signals. On the other hand, positively charged residues in the cytoplasmic domain are not required for signal or anchoring function.

The possibility for signal sequences to act in some cases as anchoring sequences and for anchoring sequences to act as signals for translocation justify serious doubts expressed elsewhere (Etémadi, 1986a) concerning the validity of an experimental procedure used (see for example Friedlander and Blobel, 1985; Dalbey and Wickner, 1988) to show the existence of internal signal sequences and stop transfer sequences in a protein crossing the membrane thickness more than once. Indeed in this procedure the behavior of segments outside their natural location is assayed, which can be misleading. The point was recently recognized by Audigier et al., 1987). The problem raised (Etémadi, 1986a) is of great theoretical importance. Since to date it remains uncertain whether recurrent use of signal recognition machinery is required for insertion of proteins crossing the membrane thickness several-fold. It is conceivable that once the precursor has been correctly guided and attached to the membrane and the translocation has begun, completion of insertion would follow without the necessity of further recognition events involving the recurrent use of SRP, at least when very short cytoplasmic loops exist.

\subsubsection{The Nature of the Involvement of the Mature Part of Exported Proteins in the Translocation Process}

The question can be raised as to the requirement for parts of the molecule other than the signal sequence in the translocation. Present data indicate that for most natural secretory and transmembrane integral proteins a functional signal is sufficient. However, when a cytoplasmic protein in a hybrid precursor is attached to a signal sequence, depending on the nature of the cytoplasmic protein, it can or cannot be translocated. In several experiments (Bassford et al., 1979; Emr and Silhavy, 1982; Emr et al., 1980; Kadonaga et al., 1984; Rothstein et al., 1985), translocation of hybrid proteins did not occur correctly. The report that the $\mathrm{C}$ terminus of a secretory protein was required for its trans- 
location (Koshland and Botstein, 1980) was not confirmed (Ito et al., 1981; Koshland and Botstein, 1982; Pollit and Zalkin, 1983). However, other reports were interpreted as favoring the requirement of segments of the mature part of the exported molecules in the process of translocation (Bankaitis and Bassford, 1985b; Bankaitis et al., 1984; Rasmussen and Silhavy, 1987; Ryan et al., 1986). Nevertheless, these observations might find alternative interpretations (see Etémadi, 1986a); in particular, a possible change of protein conformation can also explain the lack of translocation of proteins in which segments of the mature part were deleted. Conversely, a substitution in the mature part may retard folding and allow a partially defective signal sequence to act more efficiently. A recent report by Cover et al. (1987) furnishes an illustrative example in this respect. Also, studies on hybrid proteins constructed between the wild type or mutant presequence of lipoprotein, an $E$. coli outer membrane protein, and $\beta$ lactamase are remarkable (see Ghrayeb et al., 1983, 1985; Lunn and Inouye, 1987). The efficiencies of translocation and processing were observed to be influenced by the mature parts. Such effects are presumably induced through conformational effects. Recently, McIntyre et al. (1987), working on translocation of the $E$. coli outer membrane protein OmpA and hybrids constructed of the $\mathrm{N}$ terminus of the precursor of this protein at different sites of which were linked a 253 amino acid-long fragment of the $E$. coli phage $\mathrm{T}_{4}$ long tail fibers, arrived at the general conclusion that the export incompatibility of the soluble proteins might be responsible for cases of the inability of the signal sequences to facilitate export; that is, segments of the mature part of exported proteins are not required in triggering the translocation process. A remarkable case, reported by Fitts et al. (1987), discusses the secretion of defective mutants of $\beta$-lactamase in $S$. typhimurium. In this case mutation consists of substitution of tyrosine for cysteine in the mature part of the protein. Here, however, translocation and processing occurred but the protein remained bound to the outer aspect of the inner membrane.

\subsection{Signal Sequences for Migratory Proteins}

\subsubsection{Location and Structural Features of Signal Sequences of Migratory Proteins}

Involvement of extrasequences of mitochondrial migratory proteins in their uptake is supported by the fact that when mitochondrial and cytoplasmic isoenzymes exist, only mitochondrial forms have a precursor endowed with an extrasequence, even in cases in which both forms are encoded by a single gene, as is the case for the two forms of histidine tRNA synthetase (Natsoulis et al., 1986) in S. cerevisiae. When isoenzymes are encoded by distinct genes, as is the case for yeast alcohol dehydrogenases (Young and Pilgrim, 1985) or citrate 
synthetases (Rosenkrantz et al., 1986), only those coding for organellar isoenzymes provide an extrasequence. Note that such observations concerning natural products, which point to the high significance of $N$-terminal signals for translocation processes, can be extended to the example of a protein translocated through the endoplasmic reticulum. Carlson and Botstein (1982) have found that in $S$. cerevisiae the single gene for invertase encodes two mRNAs, one providing a full signal sequence for the secreted enzyme and the other for the intracellular enzyme.

Numerous observations indicate that extrasequences of migratory proteins, when present, are $\mathrm{N}$ terminal and their length much more variable than that of signal sequences of secretory and transmembrane integral proteins (see Table II). Recently, the involvement of the $N$-terminal extra-peptide in mitochondrial protein translocation was confirmed by Sakakibara et al. (1987) by observing that an antibody raised to the 29 residue-long signal of rat liver aspartate aminotransferase inhibited the import of the precursor into rat liver mitochondria. As we shall see, however, in rare cases the presence of an internal signal and, in the case of microbody proteins, the occurence of $\mathrm{C}$-terminal signals are reported. Extra-sequences of migratory proteins contrarily to those of secretory and transmembrane integral proteins are generally devoid of a hydrophobic stretch, while being rich in basic amino acid residues. In fact, with some exceptions, such as the case of rat liver aspratate aminotransferase (Kamisaki et al., 1982; see however Sakakihara et al., 1987)—-though not that of chicken liver enzyme (Jaussi et al., 1985) - and the case of a subunit of 17,000 Da of the yeast complex III of electron transport system (Van Loon et al., 1984), extrasequences of mitochondrial imported proteins were generally found to be basic. Several mitochondrial migratory proteins are devoid of a cleaved signal sequence (De haan et al., 1983; Hampsey et al., 1983; Mori et al., 1985; Nakagawa et al., 1987; Teintze et al., 1982; Watanabe and Kubo, 1982; Zimmermann and Neupert, 1980a). The signal in such cases might be $\mathrm{N}$-terminal but uncleaved or internal. In one such case, that of a 14,000 Da subunit of yeast mitochondrial complex III of electron transport, the $\mathrm{N}$ terminus of the protein was found to be rich in basic residues (see Table II) (De Haan et al., 1983; see also Nakagawa et al., 1987). In the case of 3-ketoacylCoA thiolase, a matrix enzyme, the presence of an internal sequence was envisaged (Mori et al., 1985). However, more recently, the location of the signal at the $\mathrm{N}$ terminus was not excluded (Arakawa et al., 1987). Adrian et al. (1986), using gene fusion techniques, found that for the case of a mitochondrial inner membrane protein, ADP-ATP carrier, which is also devoid of a cleaved extra-sequence, the information targeting the precursor to mitochondria was present in the first 115 residues of the molecule (out of 309 amino acid residues), in addition the amino terminal sequences prevented the membrane anchoring sequence (residues 78 to 98) from stopping transfer through the outer membrane. However, in a recent work, Pfanner et al. (1987c) have reported that a truncated ADP/ATP 


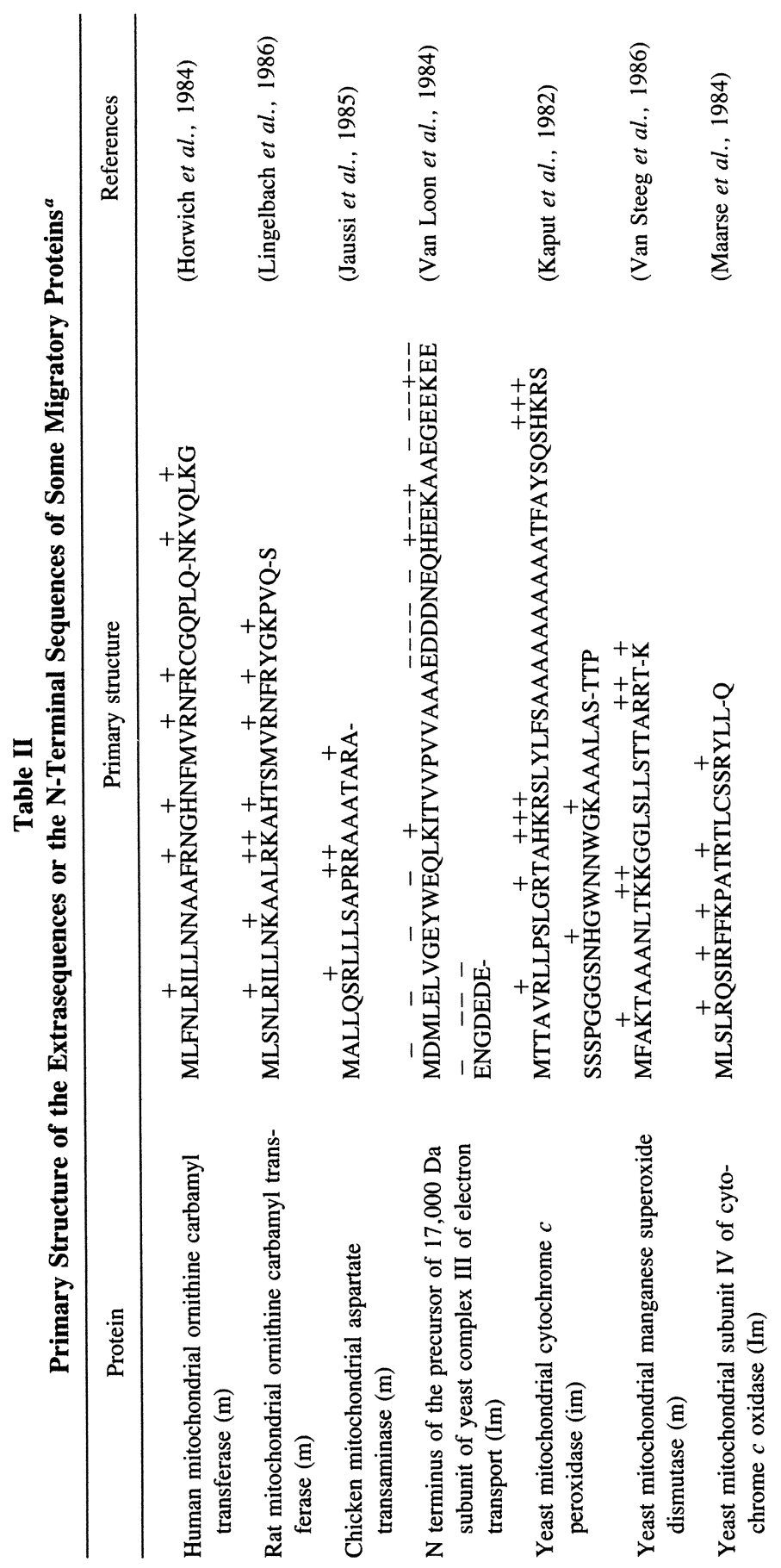




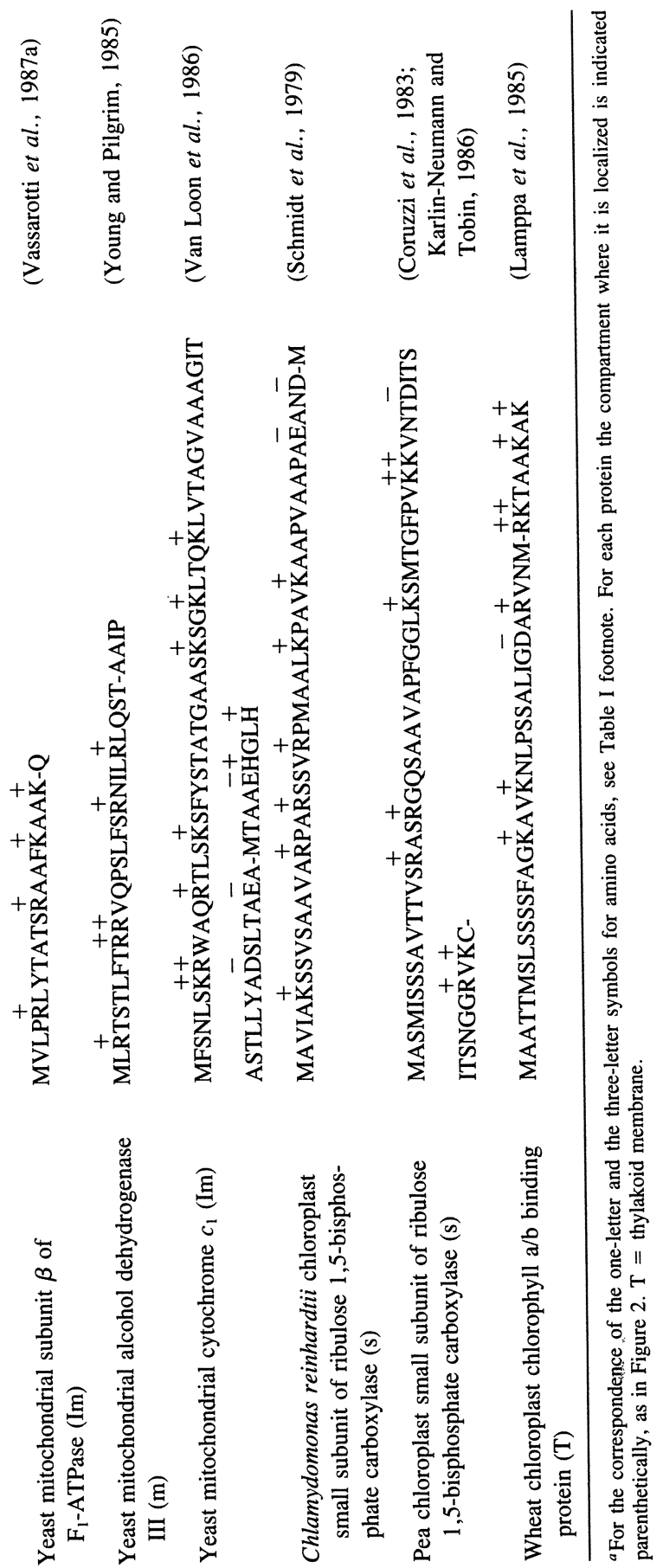


carrier lacking $103 \mathrm{~N}$-terminal residues was able to be imported in mitochondria in a receptor- and energy-requiring manner. Nevertheless, for reasons mentioned repeatedly in the text, in such situations it is advisable to check the inefficiency of the $N$-terminal parts of the molecule.

The richness in basic residues was first suggested to be of importance for interaction of the precursor of a chloroplast migratory protein, ribulose 1,5bisphosphate carboxylase, with chloroplasts (Schmidt et al., 1979; see also Cashmore, 1983; Lamppa et al., 1985) and then for precursors of numerous mitochondrial proteins with mitochondria (Horwich et al., 1984; Hurt et al., 1984; Kaput et al., 1982; Kumamoto et al., 1987; Maarse et al., 1984; Miura et al., 1982; Mori et al., 1982; Viebrock et al., 1982; see also Nagata et al., 1983; Yoshida et al., 1985). Several observations indicate that positively charged rhodamines, rhodamine 123 (Anderson, 1981; Mori et al., 1982; Morita et al., 1982; Oda et al., 1984) and rhodamine 6G (Horwich et al., 1985c), prevent import of mitochondrial proteins. Such observations were interpreted in terms of an action at the level of binding of positively charged precursors to negative charges on the mitochondrial membrane (Morita et al., 1982). Rhodamine 123 (Johnson et al., 1981) and its ethyl analogue (Severina and Skulachev, 1984) are vital dyes that also penetrate into the matrix of mitochondria and the cytoplasmic compartment of bacteria. Some authors (see Lubin et al., 1987) do not exclude interaction of rhodamine 123 with mitochondrial membrane lipids.

Studies by Horwich et al. (1985a) in which a mitochondrial protein, ornithine carbamyl transferase, was allowed to be synthesized in the presence of canavanine instead of arginine in HeLa cells transfected with a plasmid containing the cDNA for mRNA of the enzyme indicated that the four arginine residues present in the extrasequence of 32 residues (see Table II) are required for import of the precursor. Also, in vitro experiments indicated that the precursor in which three distal arginine residues were replaced by glycine could not be imported (Horwich et al., 1985b). Besides the basicity, emphasis is placed on the role of the secondary structure of the extrasequence in the process of binding and import. Horwich et al. $(1986,1987)$ found that deletions at the $\mathrm{N}$ and $\mathrm{C}$ termini of the extrasequence did not affect translocation of ornithine carbamyl transferase, while the middle portion was absolutely required. Furthermore, the presence of arginine residue at position 23 was of importance; its substitution by glycine led to a complete loss of translocation. Arg 23 was suggested to contribute to a local secondary structure, presumably $\alpha$-helical, which would be essential for the function in the midpoint of the extension sequence. Further work (Sztul et al., 1987), however, indicates that substitution of either aspartate or glycine for Arg 23 did not prevent translocation and processing which normally occurs in two steps (first an intermediate is formed in which the cleavage occurs between residues 24 and 25 , then the further eight residues of the extra-sequences are removed). Such substitutions revealed an- 
other "cryptic" site of cleavage between residues 16 and 17 . Remarkably enough residue 15 of the extra-sequence is also an arginine. Lingelbach et al. (1986), having observed that residues 15-19 of the extrapeptide of the same protein, including positively charged residues, could be deleted without the function of the signal being affected, suggested that "specific structural elements containing basic residues" are involved rather than the general basicity of the signal. Chu et al. (1987b), working on translocation of rat mitochondrial malate dehydrogenase precursor mutated in the region of the extra-sequence, confirmed a role for the positive charge (of arginine residues) in enhancing the efficiency of translocation. Interestingly, however, the authors (Chu et al., 1987a) found also that the uncharged residue leucine at position 13 of transit sequences plays an important role in the binding of the precursor to mitochondria, hence in the import. Recently, Vassarotti et al. (1987b) reported that subrogate signals in the $N$-terminal mature part of mitochondrial $F_{1}$-ATPase $\beta$-subunit could be generated by spontaneous mutations. The subunit, the import of which was prevented by deletion of the signal, was then imported. Effective mutations replaced different acidic residues with neutral and basic residues and generated amphiphilic helices with a less acidic character. It appeared that while an amphiphilic helix at the $\mathrm{N}$ terminus containing a minimum basic residues is required, all subrogate signals were acidic. They showed a reduced in vivo efficiency and an inefficient but detectable in vitro import-mediating capability.

In contrast to the case of ornithine carbamyl transferase (Horwich et al., 1986, 1987), there are reports indicating that only an $\mathrm{N}$-terminal segment of the extrasequence of mitochondrial imported proteins with a sufficient length can induce uptake. Thus, Vassarotti et al. (1987a) report that deletion of residues $10-36$ of the precursor of $\beta$-subunit of yeast mitochondrial $F_{1}$-ATPase does not affect import and assembly into a functional enzyme. At least $10 \mathrm{~N}$ terminal residues out of the 19-residue transit sequence (see Table II) were required for the translocation to occur. Various observations on the inhibition (Furuya et al., 1987; Gillespie et al., 1985) by synthetic peptides, representing $\mathrm{N}$-terminal segments of extra-sequences of mitochondrial migratory proteins, of the import of precursors of these proteins also favor involvement of their $\mathrm{N}$ termini in the translocation process (see also Section 6.2).

Application of the gene fusion technique confirmed the crucial function of extrasequences of mitochondrial and chloroplast migratory proteins and the role of their N-terminal segment. In such experiments the amino terminal sequence of a mitochondrial or chloroplastic protein is attached to a nonexportable moiety and the localization of the hybrid is examined. A chimeric gene, the product of fusion of a part of the gene for the $\beta$-subunit of yeast $F_{1}$-ATPase coding for $350 \mathrm{~N}$-terminal amino acid residues of the precursor and a large part of $E$. coli lac $\mathrm{Z}$ gene encoding the $\mathrm{C}$ terminus of the protein endowed with the enzyme activity, was found to be expressed in yeast and translocated for more than 
90\%. However, the respiration was blocked, presumably because of jamming of the hybrid protein at a terminal step during the transit (Douglas et al., 1984). In further such experiments, Emr et al. (1986) constructed hybrids of yeast $\beta$ subunit and either the $E$. coli lac $\mathrm{Z}$ gene product ( $\beta$-galactosidase) or the yeast suc 2 gene product (invertase). Such hybrids were imported in vivo into mitochondria in yeast. These and experiments using defective $\beta$-subunit gene deletion mutants indicated that 27 amino acids on the $\mathrm{N}$ terminus of the precursor were sufficient for import of a soluble protein, though the exact location of imported proteins was not elucidated. Other studies have indicated that a segment of 53 residues at the $\mathrm{N}$ terminus of the subunit IV of yeast cytochrome $c$ oxidase (including the extrasequence of 25 residues) (Table II) was sufficient for targeting the cytosolic protein, dihydrofolate reductase, into mitochondria (Hurt et al., 1984). Likewise, fusion experiments indicated that the extrasequence of the matrix enzyme ornithine carbamyl transferase could target dihydrofolate reductase into mitochondrial matrix in transfected $\mathrm{CHO}$ cells (Horwich et al., 1985c). For certain mitochondrial proteins such as carbamylphosphate synthase I $\left(M_{\mathrm{r}}=165,000\right)$, the in vitro translocation into isolated mitochondria is very slow. However, here again, hybrid protein experiments indicate involvement of the extrasequence: a hybrid bearing the extrasequence of 38 amino acid residues of the enzyme (Table II), 55 amino acids at the $\mathrm{N}$ terminus of its mature part, and the carboxyl terminal 209 amino acids of the ornithine carbamyl transferase was imported and processed in isolated mitochondria (Nguyen et al., 1986). More recently, Nguyen and Shore (1987) observed translocation into mitochondria of a hybrid constructed by cDNA fusion from the signal of preornithine carbamyl trasnferase and the $C$ terminus of the $G$ protein of the envelope of VSV. The protein was inserted into the inner membrane, presumably in a transmembrane manner. Van Loon and Young (1986), working on three alcohol dehydrogenase isoenzymes of yeast, observed an 80-90\% identity of sequences between the two cytosolic and the single mitochondrial forms. The last enzyme has a 27 residue long extrasequence (see Table II). When by the gene fusion technique the $\mathrm{N}$-terminal segment of 48 residues of the mitochondrial form replaced $21 \mathrm{~N}$-terminal residues of the cytosolic forms, these were translocated to the mitochondria, but when the first $\mathrm{N}$-terminal 28 residues of the mitochondrial signal were deleted, the hybrid proteins could not be translocated.

As for mitochondrial imported proteins, extrasequences of those of chloroplasts are able to target soluble proteins into these organelles. Thus, the extrasequence of the small subunit of ribulose 1,5-bisphosphate carboxylase linked to various soluble proteins targeted these proteins to the stroma (Boutry et al., 1987; Kuntz et al., 1987; Schreier et al., 1985; Van Den Broek et al., 1985; Wasmann et al., 1987). During these experiments it was observed that large 
deletions in the amino terminal or the central part of the extrasequence strongly reduced the translocation (Wasmann et al., 1987). Also, when the hybrid contained only $41 \mathrm{~N}$ termini out of the 57 residues of the signal, it remained mostly cytoplasmic, bound to the outer membrane, although its $\mathrm{N}$ terminus reached the stroma and a segment of it was cleaved (Kuntz et al., 1987). Della-Cioppa et al., (1987) report that the extrasequence of petunia chloroplast enzyme 5enolpyruvylshikimate-3-phosphate synthase can import the $E$. coli soluble enzyme into coloroplasts. The experiment is of potential agronomical interest since the plant enzyme involved in production of aromatic amino acid is blocked by herbicides in which glyphosate is the active element, while the bacterial enzyme activity is not affected.

In remarkable experiments, Hurt et al. (1986a) observed that the extrasequence of a mitochondrial protein could target both the large and the small subunits of chloroplast ribulose 1,5-bisphosphate carboxylase to mitochondria. Surprisingly, however, Hurt et al. (1986b) also report that the 31 amino terminal residues of the extrasequence of the small subunit of ribulose 1,5-bisphosphate carboxylase could direct mouse dihydrofolate reductase and the mature part of the subunit IV of the mitochondrial cytochrome $c$ oxidase into the matrix and the inner membrane of isolated yeast mitochondria, respectively. This result raises the problem of the mechanism of discrimination of proteins targeted to chloroplasts and mitochondria in plants and, in fact, other authors (Boutry et al., 1987) have recently reported that in a transgenic plant (Nicotiana plumbaginifolia) the transit sequence of the small subunit of ribulose 1,5bisphosphate carboxylase directs a soluble $E$. coli enzyme into chloroplasts, while the same bacterial enzyme (chloramphenicol acetyltransferase) synthesized in a hybrid protein comprising $\mathrm{N}$-terminally a sequence of 90 amino acids of the $N$-terminal end of the $\beta$-subunit of the mitochondrial ATP synthase was directed to the mitochondria of the transgenic plant cells. Furthermore a recent report by Smeekens et al. (1987) indicates that chimeric precursor proteins comprising the transit peptide of chloroplast proteins, ferredoxin, a stromal protein; or plastocyanin, an intrathylakoidal luminal protein, and the mitochondrial manganese superoxide dismutase chain, are imported to the chloroplast stroma. Such chimeric proteins were not taken up by yeast mitochondria.

Reports by Baker and Schatz (1987) and Hurt and Schatz (1987) indicate that sequences in prokaryotic or eukaryotic genome, even those directing the synthesis of segments of soluble proteins when fused to the genome of a migratory protein truncated from the part of it coding for the presequence, are able to target passenger proteins and to mediate their import into mitochondria. Such reports confirm similar observations with secretory proteins mentioned above and are in line with the previous observation of Lingappa et al. (1979) that a segment of ovalbumin, residues 234-253, having nothing to do with the 
signal sequence of this protein, which is located near the $\mathrm{N}$ terminus of the molecules (Austen, 1979; Meek et al., 1982; Tabe et al., 1984), could inhibit import of some secretory proteins in competitive translocation experiments.

In the case of microbodies, matrix proteins are reported to be generally devoid of an extrasequence (Geredes et al., 1982; Goldman and Blobel, 1978; Goodman, 1985; Goodman et al., 1984; Kruse and Kindl, 1983; Lord, 1978; Lord and Roberts, 1982; Maechina et al., 1988; Osumi et al., 1985; Roa and Blobel, 1983; Robbi and Lazarow, 1978; Roggenkamp et al., 1984; Zimmermann and Neupert, 1980b). However, there are cases for which the presence of an extrasequence is mentioned (Arakawa et al., 1987; Furuta et al., 1982; Hock and Gietl, 1982; Miura et al., 1984; Riezman et al., 1980; Sautter et al., 1987; Walk and Hock, 1978; Yamaguchi et al., 1986, 1987). In one such case, that of germinating pumpkin glyoxysomal malate dehydrogenase of $33,000 \mathrm{Da}$, the extrasequence was found to be rapidly cleaved from the precursor of 38,000 $\mathrm{Da}$ upon its translocation, as revealed by pulse-chase experiments, so that while the precursor was cytoplasmic, only the mature form was found in the organelle (Yamaguchi et al., 1987). However, in another recent report concerning Candida tropicalis peroxisomal acyl-CoA oxidase, Small and Lazarow (1987) indicate that only $40 \%$ of the carboxy terminal of the molecule had sufficient information to be, in part, imported in peroxisomes as revealed by protease protection. Nevertheless, further work is required to substantiate the interpretation that truncated proteins were in fact in the matrix and in any event the possibility cannot be excluded that an $\mathrm{N}$ terminus might still be a more efficient targeting segment in the wild-type molecule. Gould et al. (1987) admit the involvement of a C-terminal segment of 12 amino acids of the firefly luciferase in its targeting and translocation to peroxisomes. In addition, however, another region contained in amino acids 47 to 261 was implicated. Swinkles et al. (1988), comparing genes for cytosolic and glycosomal phosphoglycerate kinase of Crithidia fasciculata, conclude that a C-terminal extension may be involved in targeting of the microbody enzyme. A similar situation would also prevail for the Trypanosoma brucei enzyme.

\subsubsection{Signals for Translocation to Different Organellar Compartments}

The "topogenic sequence hypothesis" proposed the presence of different signal sequences and specific receptor translocators (Blobel, 1980) to explain how different organellar proteins reach different compartments. However, a discrimination for proteins of different compartments at the level of the initial interaction of percursors and membrane receptors according to their destination in mitochondrial compartments does not seem to occur. Gillepsie et al. (1985) have found that a peptide corresponding to the amino terminal residues 1-27 of the precursor of ornithine carbamyl transferase, a matrix protein, prevented 
not only the uptake of the corresponding precursor but also that of a mitochondrial inner membrane protein. Also Furuya et al. (1987) report that N-terminal sequences (residues 1 to 15 or 1 to 20 of the 39-residue-long extra-sequence) of cytochrome P-450 scc prevent the import of some mitochondrial proteins of the matrix or the inner membrane. The same effect was observed with residues 1 to 14 of the $\mathrm{N}$-terminal of adrenodoxin. As reported for some proteins of intermembrane compartment of mitochondria such as cytochrome $b_{2}$ (Gasser $e t$ al., 1982b; Ohashi et al., 1982) and cytochrome $c$ peroxidase (Kaput et al., 1982; Reid et al., 1982), the precursors are first linked to the inner membrane, then subsequently processed and released in the intermembrane compartment. A theory was recently suggested to explain import of mitochondrial proteins into different compartments (Hurt and Van Loon, 1986). It is based on observations indicating that whole extrasequences are not always required for the uptake. Thus, proteins formed of the extrapeptide of the subunit IV of complex III of the electron transport system or of only the first 12 amino acids at its $\mathrm{N}$ terminus and of mouse dihydrofolate reductase were found to be targeted to the matrix of isolated yeast mitochondria in vitro and also during in vivo experiments. The N-terminal segment of less than nine residues was not sufficient (Hurt et al., 1985). More recently, Van Loon et al. $(1986,1987)$ reported that the only $16 \mathrm{~N}$-terminal (or less) residues of the extrasequence of cytochrome $c_{1}$ were sufficient for targeting a passenger protein to the matrix and that $13 \mathrm{C}$ terminal residues of the signal acted as a stop transfer sequence anchoring the protein (or a hybrid comprising the presequence and the dihydrofolate reductase) in the inner membrane. Also, only the nine N-terminal amino acid residues of $\delta$-aminolevulinate, a matrix enzyme, linked to $\beta$-galactosidase, were able to import the hybrid into mitochondria. In this case the precursor is of 61,000 and the mature enzyme of 58,000 Da; hence the extrasequence should be of some $2000 \mathrm{Da}$ (Keng et al., 1986). Remarkably, the $\mathrm{N}$ terminus of an outer membrane protein of $70,000 \mathrm{Da}$ was found to be involved in targeting and anchoring the protein (Riezman et al., 1983b). Only $41 \mathrm{~N}$-terminal residues were sufficient for targeting and anchoring hybrids constructed with $E$. coli $\beta$-galactosidase. The targeting would be mediated by a region including the 11 amino terminal residues and anchoring a stretch of uncharged segment between residues 9 and 38 (Hase et al., 1984). A hybrid, comprising 21 amino terminal residues and expressed in yeast, was localized in the matrix compartment, hence the suggestion that common targeting sequences for outer membrane proteins and at least some matrix proteins exist (Hase et al., 1986). Such experiments led to the generalization (Hurt and Van Loon, 1986) that amino terminal sequences of mitochondrial proteins generally possess an $\mathrm{N}$-terminal "matrix targeting domain" containing a periodic array of basic amino acids involved in both attachment and transport to the matrix. Membrane-bound proteins would then have a stop transfer sequence. A long stop transfer domain 
with an uninterrupted stretch of uncharged amino acid residues, followed by charged residues, prevents transport through the outer or the inner membrane. The protein becomes an outer membrane protein or inner membrane protein (Figure 4). The presence, in addition, of cleavage sites would ensure that part or all of the presequence is cleaved. An intermembrane space protein is the product of cleavage of a protein first bound to the inner membrane. Further work, however, is required to understand details of translocation of proteins to different compartments. A recent proposal by Hartl et al. (1987a) for the path of translocation of an intermembrane protein, Rieseke FeS protein of the com-

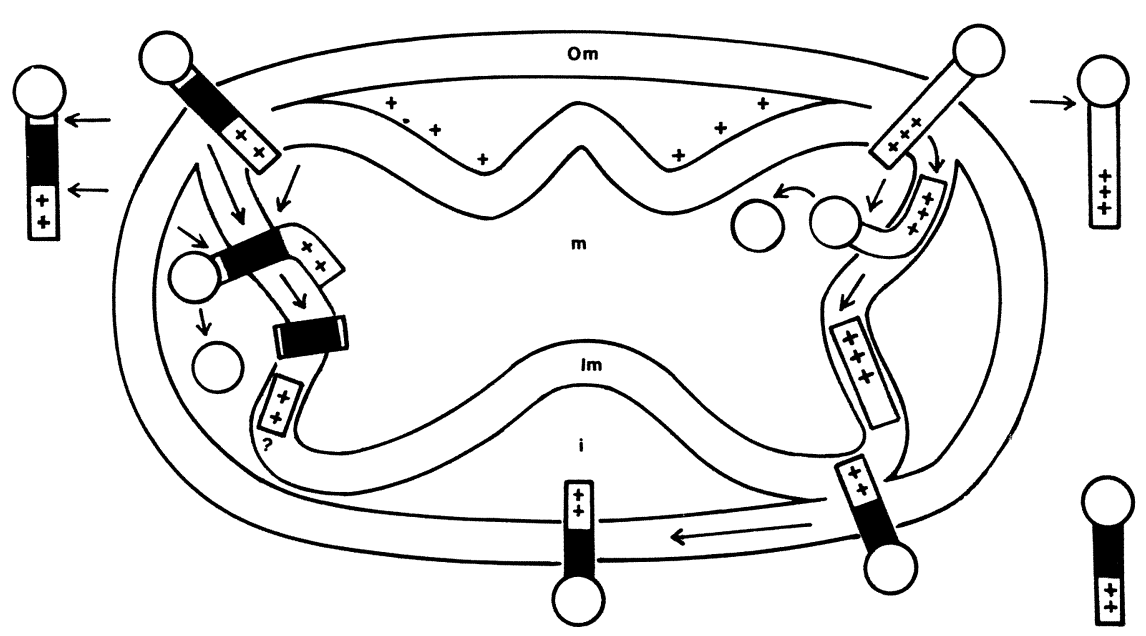

FIGURE 4. Translocation through the mitochondrial membranes of a matrix and an intermembrane compartment protein and insertion of an outer membrane protein are schematized (see Hurt and Van Loon, 1986). It is suggested that all proteins destined to mitochondria have an N-terminal segment able to target proteins to mitochondria and a "passenger" part. The mitochondria-targeting segments have in all cases a positively charged matrix-targeting segment at their $\mathrm{N}$ termini. In the upper right side, this matrix-targeting segment is shown followed by the passenger, appearing as a plain disc. The cleavage site of the extra sequence is indicated (arrow). The upper left drawing shows translocation and processing of the precursor of a protein destined to the intermembrane compartment (i). Such a precursor would exhibit a matrix-targeting segment, shown as a positively charged rectangle, then a segment shown as a dark rectangle, anchoring the molecule in the inner membrane. Two cleavage sites on the extra sequence exist, the matrix-targeting segment would be cleaved in the matrix and the second segment in the intermembrane compartment. For an inner membrane protein exposed to the intermembrane space, this second cleavage does not occur. In the lower right side a protein destined to the outer mitochondrial membrane is shown. This protein has, in addition to the matrix-targeting positively charged segment, an outer membrane-targeting sement shown as a dark surface. It is important to note that, for all cases, translocation through mitochondrial membranes of proteins of different compartments starts by that of their $\mathrm{N}$ termini. Cleavage sites are shown by arrows. Different compartments are located as in Figure 2. 


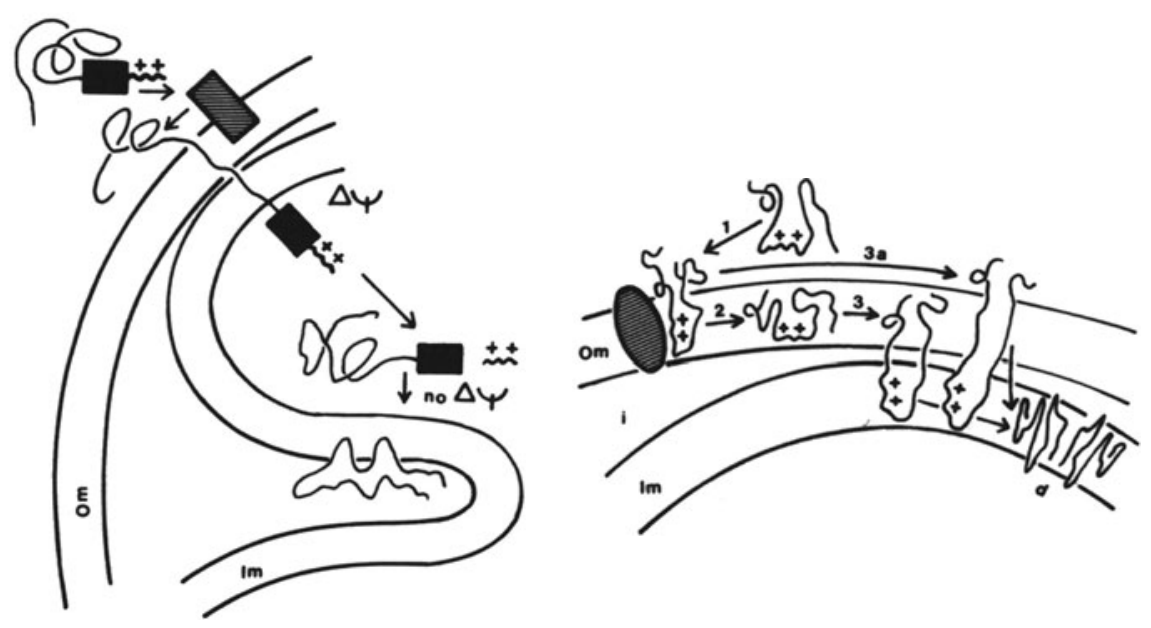

FIGURE 5. Mechanisms for incorporation of a mitochondrial intermembrane compartment protein, Rieske iron-sulfur protein (left side drawing), and of an inner membrane protein ADP-ATP carrier (right side drawing) are shown (see Hartl et al., 1987; Pfanner and Neupert, 1987). Incorporation of the precursor of iron-sulfur protein, which is cleaved of two segments from its $\mathrm{N}$ terminus indicated as a positively charged segment and a dark rectangle, involves interaction first with a membrane receptor (hatched rectangle). The precursor passes, at $25^{\circ} \mathrm{C}$ and in the presence of the membrane potential $(\Delta \psi)$, through contact zones between the two mitochondrial membranes and is released in the matrix compartment where the first segment (shown with positive charges) is cleaved. The intermediate is formed in the matrix compartment and is then translocated into the intermembrane space and cleaved without requirement for $\Delta \psi$. Translocation of ADP-ATP carrier, which is devoid of a processed signal sequence, is also dependent on $\Delta \psi$; the protein interacts with a membrane receptor (striated ellipse) in order to bind specifically to the membrane (reaction 1) and at $25^{\circ} \mathrm{C}$ inserts into the outer membrane (reaction 2). In the presence of $\Delta \psi$ the protein passes through an intermediate stage (reaction 3 ) and reaches the inner membrane where it forms the dimer (d). Passage occurs through the contact zones between the two mitochondrial membranes. The initial binding to the receptor can occur at low temperature and, in the presence of $\Delta \psi$, an essential event of translocation takes place (reaction 3a). The protein on the one hand has reached the inner membrane and on the other hand is still exposed at the mitochondrial suface. Raising the temperature to $25^{\circ} \mathrm{C}$ leads to completion of translocation to the inner membrane and formation of the dimer (d).

plex III (Figure 5), does not comply with the above model. This protein is peripherally bound to the outer leaflet of the inner membrane. Its precursor was found to be entirely translocated into the matrix. Processing occurs in two steps with formation of an intermediate which also was located in the matrix. Van Loon and Schatz (1987) have confirmed the occurrence of the last transport pathway for iron sulfur protein. Hartl et al. (1987b) proposed that the hydrophobic stretch in the precursor does not act as stop transfer sequence but is used instead after the first cleavage in the matrix for the targeting of the inter- 
mediate to and its translocation through the inner membrane; cleavage of this sequence occurs in the intermembrane compartment. Also shown in Fig. 5 is the route taken by ADP-ATP carrier protein, an inner membrane protein, details of which do not comply with the above model.

In the case of chloroplasts, proteins imported to the stroma have to cross the two membranes of the envelope. In a further step, thylakoid membrane proteins would then have to be membrane inserted and intrathylakoid luminal compartment proteins would have to cross through the thylakoid membrane. There are reports indicating that extrasequences of migratory proteins of chloroplasts might have specific domains responsible for their correct targeting to different organellar compartments. Thus, Smeekens et al. (1986) have found that precursors of ferredoxin, a stroma protein, and plastocyanin, an intrathylakoid luminal protein of white campion (Silene pratensis), could be incorporated in vitro into pea chloroplasts. When chimeric cDNAs were constructed in which transit sequences of these two proteins were exchanged and import into isolated chloroplasts of in vitro synthesized hybrids was examined, ferredoxin transit peptide directed plastocyanin to the stroma. Plastocyanin transit peptide led to a processing intermediate that was arrested on its way to the lumen. A "two-domain hypothesis" was suggested, which states that the plastocyanin transit peptide comprises two domains, the first functioning in the import of proteins to chloroplasts and the second in transport across the thylakoid membrane. In the case of two luminally located thylakoid membrane proteins bound peripherally to the membrane, a two-step cleavage, one related to their passage through the envelope and another with the crossing of the thylakoid membrane, is proposed (Chia and Arntzen, 1986). Recently Kirwin et al. (1987) confirmed the two step maturation of plastocyanin and partially purified the thylakoid peptidase responsible for the second maturation step. In further studies Smeeken et al. (1987) observed that a chimeric precursor protein comprising the extrasequence of plastocyanin and the sequence of the yeast mitochondrial manganese superoxide dismutase is taken up by chloroplasts and remains in the stromal compartment in an intermediate state in which only the signal for the import into chloroplast is cleaved. This observation reveals that while the manganese superoxide dismutase section of the chimeric molecule has been imported through the two membranes of chloroplast envelope, it did not cross the thylakoid membrane. A possible explanation is that a conformational change prevents its translocation. Alternatively it might impede the correct function of the residual extra-sequence. It might also bear a segment(s) incompatible with its translocation through the thylakoid membrane.

In a recent report, Karlin-Neuman and Tobin (1986) identified blocks of homology in the transit sequences of light-harvesting chlorophyll $\mathrm{a} / \mathrm{b}$ protein, a protein of the thylakoid membrane, with that of the small subunit of ribulose 
1,5-bisphosphate carboxylase of the stroma compartment. Such homology blocks do not exist for signal sequences in general, including those of mitochondrial imported proteins. Located at the beginning, the middle, and the end of the extrapeptides, such sequences would constitute a common framework for a common function in protein import. Interblock segments are either dispensable or involved in import into different compartments. A recent report by Reiss $e t$ al. (1987) indicates that only the $\mathrm{N}$ - and C-termini in the transit peptide are endowed with functional importance. However, they suggest that the central part may have a role in import selectivity.

\subsubsection{The Mature Part of Migratory Proteins and the Translocation Process}

An important factor recently recognized to influence the import of migratory proteins into mitochondria is the protein conformation. Eilers and Schatz (1986) report that methotrexate, a folate antagonist, blocks import into mitochondria of a hybrid in which the dihydrofolate reductase sequence is fused to the extrasequence of a mitochondrial imported protein. It was suggested that as a result of the firm binding of the antagonist the enzyme moiety cannot be unfolded in a form compatible with translocation. More recently Vestweber and Schatz (1988) were able to confirm this idea by "destabilizing" the dihydrofolate reductase portion of the hybrid bearing the first 16 residues of the extrasequences of yeast cytochrome oxidase subunit IV N-terminally. Destabilization in these assays resulted from manipulating the sequence by site-directed mutagenesis and was assessed by the increased sensitivity towards proteases or by a decreased aptitude of binding methotrexate. Such experiments have shown that "destabilized" precursors were incorporated with an increased efficiency into membranes. The fact that precursors of cytochrome $c$ peroxidase (Kaput and Blobel, 1986) and aspartate aminotransferase (Sharma and Gehring, 1986) have to be imported as apoproteins into mitochondria are also related to the requirement for an unfolded state of precursors. Chen and Douglas (1987) observed that substitution of 129 residues from the C-terminal of the 511-aminoacid-long precursor of $\beta$-subunit of yeast $\mathrm{F}_{1}$-ATPase by yeast copper metallothionein allows the import of hybrid protein in the absence of copper. In the presence of copper, import does not occur, presumably because unfolding of the copper metallothionein part is prevented. A recent report by Ness and Weiss (1987) indicates that C-terminally truncated carbamyl phosphate synthase is imported more slowly than the full-length precursor in N. crassa mitochondria. It can be envisaged that, here again, an unfavorable abnormal conformation might intervene. The authors mention the alternative possibility of an involvement at the level of receptor-signal interaction. Van Steeg et al. (1986) have 
found that when a sequence of 34 amino acid residues, 26 of which constitute the extrasequence of the matrix enzyme manganese superoxide dismutase of yeast (Table II), was fused to the cytosolic yeast enzyme invertase, import into isolated mitochondria of the fusion protein did not occur and only a low binding to mitochondria was observed. Also, in yeast transformants bearing the fusion gene, some $95 \%$ of the hybrid protein remained cytoplasmic, the remainder being loosely attached to the inner membrane. Presumably, either folded domains of invertase obstruct the passage, or the membrane jamming results from passage of some hybrid molecules. Likewise, a chimeric protein comprising the extrasequence of the small subunit of ribulose 1,5-bisphosphate carboxylase, $13 \mathrm{~N}$-terminal amino acids of the mature part of the enzyme, and in addition the sequence of a cytoplasmic protein (the so-called shock protein) was largely not imported. It was concluded that "a transit peptide alone may not be sufficient for efficient import" (Lubben and Keegstra, 1986). In other experiments, however, it was found that although the transit peptide of the small subunit of ribulose 1,5-bisphosphate carboxylase is sufficient for triggering translocation of the hybrid in which it was linked $\mathrm{N}$ terminally to neomycin phosphotransferase, a soluble cytoplasmic protein (Kuntz et al., 1987; Wasmann et al., 1987) the presence, in addition, of the $23 \mathrm{~N}$-terminal residues of the mature part of the subunit favorably affected the translocation. This additional sequence would intervene through an alteration of conformation improving the recognition by the receptor (Wasmann et al., 1987). Nonetheless, others found that in the presence of these 23 residues chloroplasts would accumulate less of the hybrid, because of a competition between the rate of its uptake and that of its cytoplasmic degradation (Kuntz et al., 1987). Experiments made by Gearing and Nagley (1986) still point to a factor additional to the conformation. In these experiments a 40-amino-acid-long $\mathrm{N}$-terminal segment of the precursor of subunit IV of cytochrome $c$ oxidase, linked to subunit 8 of yeast mitochondrial ATP synthase, a highly hydrophobic protein, was unable to induce import of this protein into yeast mitochondria; while the extrasequence of the subunit 9 of $N$. crassa $\mathrm{F}_{0}-\mathrm{F}_{1}$ ATPase of 66 amino acids bound to the same subunit 8 could trigger the import and processing of the hybrid. In this interesting case, the highly hydrophobic protein with a tendency to interact with the membrane prevents a correct import unless bound to the long hydrophilic presequences of the subunit 9.

Finally a recent report by Pfanner et al. (1987d) suggested that, in addition to the targeting $\mathrm{N}$-terminal extra-sequence, the hydrophobic, mature part, of the subunit 9 along with hydrophobic segments in some other mitochondrial imported proteins are functionally involved in the initial $\Delta \Psi$-independent binding, so that these proteins can be imported into mitochondria after establishment of membrane potential. 


\section{POST-TRANSLATIONAL PROTEIN TRANSLOCATION}

\subsection{Post-Translational Translocation of Secretory and Transmembrane Integral Proteins}

Much controversy as to the co- or post-translational nature of translocation of exported proteins in bacteria exists. Besides the disputed case of bacteriophage $f_{1}\left(M_{13}\right)$ coat protein, other reports indicate the possibility of a posttranslational translocation (Garwin and Beckwith, 1982; Koshland and Botstein, 1982). Some studies also raise only the question of a strict coupling between translation and translocation (Josephson and Randall, 1981a,b; Randall, 1983). More recently, however, the problem of post-translational import of proteins in E. coli inverted inner membrane vesicles was examined (Chen et al., 1985; Mueller and Blobel, 1984; Rhoads et al., 1984) and an energy requiring post-translational import was admitted. It is worthwhile to emphasize that the in vitro feasibility of such a process does not necessarily prejudge of its occurrence in vivo, unless it is independently shown. The case of proteins translocated through or inserted into the endoplasmic reticulum furnishes good examples for this idea. As mentioned above, such a translocation was constantly observed to occur cotranslationally, except for the case of endoproteins which insert in the membrane post-translationally. The reported occurrence of post-translational insertion of the $\alpha$-subunit of $\left[\mathrm{Na}^{+}-\mathrm{K}^{+}\right]$ATPase (Hiatt et al., 1984) was rejected in principle (Caplan et al., 1986) and could not be confirmed experimentally (Caplan et al., 1986; Geering et al., 1985). However, during some in vitro experiments the possibility of a post-translational protein translocation through the microsomal membrane, although with a reduced efficiency, was observed (Caulfield et al., 1986; Mueckler and Lodish, 1986; Perara $e t$ al., 1986). In these cases, generally the post-translational translocation occurred when the chains were not released from ribosomes. On the other hand, a shortened precursor largely truncated from its C-terminal segment, even if released from ribosomes, could be translocated post-translationally (Perara et $a l ., 1986)$. The relatively low yield generally observed in these import studies, together with the fact that nascent proteins had still to be bound to ribosomes in which translation was arrested for translocation to occur and the fact that, especially with membrane proteins, the possibility of an artifactual "reconstitution" cannot be totally excluded, were among reasons for regarding a definite conclusion as to the significance of such observations to be pending. Recent work confirming the possibility of post-translational ATP-dependent translocation of a completed secretory protein pre-pro- $\alpha$-factor of yeast (Hansen et al., 1986; Rothblatt and Meyer, 1986b; Waters and Blobel, 1986) support the feasibility of such a process. Very recently Wiedmann et al. (1988) also reported 
the aptitude of microsomes from the yeast Candida maltosa to import posttranslationally the pre-pro- $\alpha$-factor and, in addition, to insert an integral membrane protein, cytochrome P-450. However, such examples remain rare among eucaryotic translocated proteins. The striking instance of pre-prolactin reported to be translocated entirely post-translationally after its reduction (Maher and Singer, 1986) could not be substantiated by Ibrahimi (1987), who was able to correlate this case with those of proteins mentioned above, in which post-translational translocation mostly concerned incompleted chains still bound to ribosomes. Nevertheless, the point made by Maher and Singer (1986), the importance of the unfolding of the mature part of molecules for translocation, remains valid and was also emphasized in several studies on procaryotic exported proteins. Randall and Hardy (1986), working on translocation of the precursor of an $E$. coli exported protein, maltose-binding protein, also suggested that the protein is valid for post-translational export as far as it has not gained its final, stably folded conformation. A direct interaction either with the membrane or with a proteinaceous factor would prevent the folding of the polypeptide before the translocation process. More recently Park et al. (1988) reported that the presence of the signal sequence modulates (retards), in vitro, the folding of precursors of maltose-binding protein and ribose-binding protein as compared to the mature proteins. Other studies recognized a form of $\beta$-lactamase which is cleaved from the signal sequence but still bound to the membrane of the $E$. coli spheroplasts; this form, in contrast with the mature periplasmic enzyme, is sensitive to trypsin, hence not in its final conformation which is not cleaved by this enzyme. Thus, the form crossing the membrane reaches the final conformation only after release (Minsky et al., 1986). Other authors also recognize a processed but still "immature" form for an outer membrane protein, OmpA protein. This form changes its conformation by interaction with lipopolysaccharide and is translocated to the outer membrane (Freudl et al., 1986). In line with the recent developments as to the requirement of an unfolded state for protein translocation, it was suggested that a postribosomal soluble factor might act as a "denaturase" (Waters et al., 1986). However, more recently, the possibility that this factor might simply correspond to a SRP-like material was raised (Fecycz and Blobel, 1987).

Further work seems to be required to unravel the exact conditions of the post-translational translocation of secretory and membrane-inserted proteins and its physiological significance, especially when the endoplasmic reticulum is involved. A point to be kept in mind is that translocation of a series of relatively short chain polypeptides (see for example Müller and Zimmermann, 1987; Rothblatt et al., 1987), which concerns marginal cases among the vast majority of exported proteins, as remarked erlier (see Etémadi, 1980a), though interesting, should be considered apart. Since translation of at least some 75 residues is required for polypeptide chains to interact with the co-translational translo- 
cation machinery, those polypeptides of lengths within this range and, a fortiori those that have been shortened still more, cannot, in principle, be co-translationally translocated. More work seems to be required to understand the translocation of such components, regarding in particular the involvement of the general translocation machinery and also with respect to energy requirement; points on which some discrepancies exist (see Ibrahimi et al., 1986; Müller and Zimmermann, 1987; Rothblatt et al., 1987).

\subsection{Post-Translational Translocation of Migratory Proteins}

The demonstration of post-translational translocation of migratory proteins, in comparison with the case of secretory and integral membrane proteins, has been facile. Both the in vivo pulse-chase experiments (Hallermeyer and Neupert, 1976; Harmey et al., 1977) and in vitro experiments of uptake of some migratory proteins (Hallermeyer et al., 1977; Harmey et al., 1976) indicated such a post-translational event. Since these reports, numerous observations on post-translational translocation of mitochondrial proteins were made (e.g., see Argan et al., 1982; Chien et al., 1984; Gasser et al., 1982b; Hernandez-Yago et al., 1983; Kolansky et al., 1982; Lewin and Norman, 1983; Mihara et al., 1982; Miralles et al., 1983; Mori et al., 1982, 1985; Morita et al., 1982; Nelson et al., 1979; Niranjan et al., 1988; Ono and Tuboi, 1986; Reid and Schatz, 1982; Sakakibara et al., 1983, 1987; Suissa and Schatz, 1982; Yamamoto et al., 1981; Yoshida et al., 1985). During such studies it could generally be ascertained that post-translationally imported proteins reach the compartment where they are normally located (Campbell et al., 1982; Gasser et al., 1982a; Kalousek et al., 1984; Oda et al., 1984; Ono and Ito, 1984a,b; Ono and Tuboi, 1986). Post-translational translocation of proteins to chloroplasts was also reported in numerous cases (Chua and Schmidt, 1979; Cline et al., 1985; Della-Cioppa et al., 1986; Grossmann et al., 1980, 1982; Highfield and Ellis, 1978; Minami et al., 1986; Pfisterer et al., 1982). Precursors to chloroplast polypeptides could not generally be detected in vivo, presumably because of their rapid import and processing. However, post-translational translocation was shown by in vitro experiments. Transfer to the correct compartment was first observed during studies on the chloroplast stroma protein, the small subunit of ribulose 1,5-bisphosphate carboxylase (Chua and Schmidt, 1978), and then extended to some other nuclearly encoded chloroplast proteins (Kohorn et al., 1986; Schmidt et al., 1985).

In the case of migratory proteins of the matrix of microbodies the posttranslational translocation also occurs (Becker et al., 1982; Desel et al., 1982; Fujiki and Lazarow, 1982, 1985; Fujiki et al., 1984; Gietl and Hock, 1984, 1986; Goodman et al., 1984; Kindl et al., 1980; Köller and Kindl, 1980; Kruse and Kindl, 1983; Lazarow and De Duve, 1973; Lazarow et al., 1982; Miura et 
al., 1984; Riezman et al., 1980; Robbi and Lazarow, 1978; Roggenkamp et al., 1984; Teintze et al., 1982; see also Crane et al., 1982a,b, 1983) as shown by pulse-chase experiments or by the observation of involvement of free ribosomes in the genesis of microbody proteins. In different instances, the in vitro uptake of migratory proteins by microbody fractions was also shown (Becker et al., 1982; Desel et al., 1982; Fujiki and Lazarow, 1982, 1985; Hock and Gietl, 1982; Kruse et al., 1981; Lazarow et al., 1982). It can be observed that in two cases in which the demonstration of an in vitro uptake failed - that of the liver peroxisomal catalase (Goldman and Blobel, 1978) and that of Ricinus communis endosperm glyoxysomal malate synthase (Lord and Roberts, 1982)canine pancreas microsomes were used in designing import experiments. The possibility remains that this material may not possess relevant receptors for the uptake of the mentioned microbody proteins.

\subsection{Bound Ribosomes and Post-Translational Translocation of Migratory Proteins}

Before the present progress in our knowledge with regard to the posttranslational translation of migratory proteins, some earlier observations with mitochondrial proteins (e.g., see Bingham and Campbell, 1972; Godinot and Lardy, 1973; Gonzalez-Cadavid and Cardova, 1972; Kawajiri et al., 1977) had indicated involvement of ribosomes bound to the endoplasmic reticulum in their genesis. Some of these observations might now require reevaluation. However, more recent data also reveal the involvement of a "rapidly sedimented endoplasmic reticulum" of rat liver mitochondria in the genesis of some migratory proteins (Cascarano et al., 1982; Montisamo et al., 1982; Parimoo et al., 1982; C. Padmanaban, personal communication). There are reports on involvement of bound ribosomes and even of ribosomes tightly bound to the endoplasmic reticulum in the genesis of mitochondrial proteins (e.g., see Heinrich et al., 1982; Nabi et al., 1983). In addition, examples can be found in the literature in which involvement of bound ribosomes, in addition to free ribosomes, in the genesis of mitochondrial migratory proteins is recognized (see Ozasa et al., 1984). In the case of chloroplasts, mention of the involvement of the rough endoplasmic reticulum in the genesis of migratory proteins is extremely limited and concerns some eukaryotic algae (Gibbs, 1979). Remarkable is also the case of Scilla non-scripta (Rodriguez-Garcia and Sievers, 1977) in which electron microscopic images can be interpreted as revealing provision of material from the endoplasmic reticulum to the outer membrane (A. Sievers, personal communication). In the case of microbody proteins, there are data in the literature indicating implication of bound ribosomes in some instances, in addition to free ribosomes (see references in Sugita et al., 1982). In the case of peroxisomal carnitine acetyl transferase, bound ribosomes synthesize part of the enzyme 
despite the fact that free polysomes were five times more active than bound ribosomes (Miyazawa et al., 1983). For the synthesis of the glyoxysomal malate synthase, the involvement of ribosomes bound to the endoplasmic reticulum was rejected in the case of cucumber (Cucumis sativus) but could not be totally excluded in the case of castor bean (Ricinus communis) (Lord and Roberts, 1982). Presently, the existence of a correlation between the endoplasmic reticulum and microbodies cannot be totally excluded with regard to provision of some membrane proteins (Lord and Roberts, 1982; see also Hashimoto, 1982) and lipids (Crane et al., 1982a; Lord and Roberts, 1982). In the case of rat liver microsomal 3-hydroxy-3-methyl glutaryl-CoA reductase, the enzyme which is known to be cotranslationally inserted in the membrane of the endoplasmic reticulum, could be immunologically localized in the matrix of peroxisomes (Keller et al., 1985, 1986; see also Zaar et al., 1986). However, the correlation between the two enzymes with regard to their biogenetic story remains to be substantiated. In rat liver, part of peroxisomal acyl/alkyl dihydroxyacetone phosphate reductase is found in microsomes, and, most significantly in cases of Zellweger cerebrohepatorenal syndrome, in which peroxisomes are absent, the enzyme accumulates in the endoplasmic reticulum (Ghosh and Hajra, 1986), i.e., in a compartment which could normally furnish lipids and some proteins to peroxisomes. Much controversy has existed about the eventual glycosylated microbody proteins. The problem is of importance and not totally solved despite the fact that recent data (Beevers and Gonzalez, 1986; Harson et al., 1983) confirm the presence of asparagine-linked carbohydrate chains in glyoxysomal proteins. If so, however, the fact would support transport of material from intracellular membrane structures to microbodies since the genesis of asparagine-linked carbohydrate chains involves enzymes present in the endoplasmic reticulum and the Golgi in both animal (Kornfeld and Kornfeld, 1985) and plant (Beevers, 1979) cells (see also Etémadi, 1986b). It is clear that the problem of the correlation between the endoplasmic reticulum and microbodies deserves further research, despite the fact that the presence of a direct continuity between these compartments reported previously (see Kartenbeck and Franke, 1974) could not be confirmed by other authors (see Gorgas, 1985; Yamamoto and Fahimi, 1987; Zaar et al., 1987).

Since translocation of migratory proteins is assumed to involve, at an early step, interaction of their signal sequences with specific receptors of receptortranslocator systems, the proposal was made (Etémadi, 1985) that for some migratory proteins the endoplasmic reticulum might have assembled operative receptors able to interact with signals of migratory proteins before chain termination. It is implied that the transmembrane component(s) of receptor-translocator system(s) in such cases can be synthesized by involvement of the endoplasmic reticulum and that assembly of eventual peripheral and/or soluble factors implicated in precursor targeting can be achieved at this level. In this 
eventuality a "loose" binding of precursors of migratory proteins to the endoplasmic reticulum as observed in some cases (Campbell et al., 1982; Kawajiri et al., 1977) might be of physiological significance. The tight binding of ribosomes involved in the genesis of these proteins (Heinrich et al., 1982; Nabi et al., 1983) would then reveal at least the start of the translocation process and/ or binding of the apolar part of extrasequences to the endoplasmic reticulum. Figure 6 gives the description of a hypothesis envisaging different possibilities of co- or post-translational binding of the precursor of a migratory protein, here

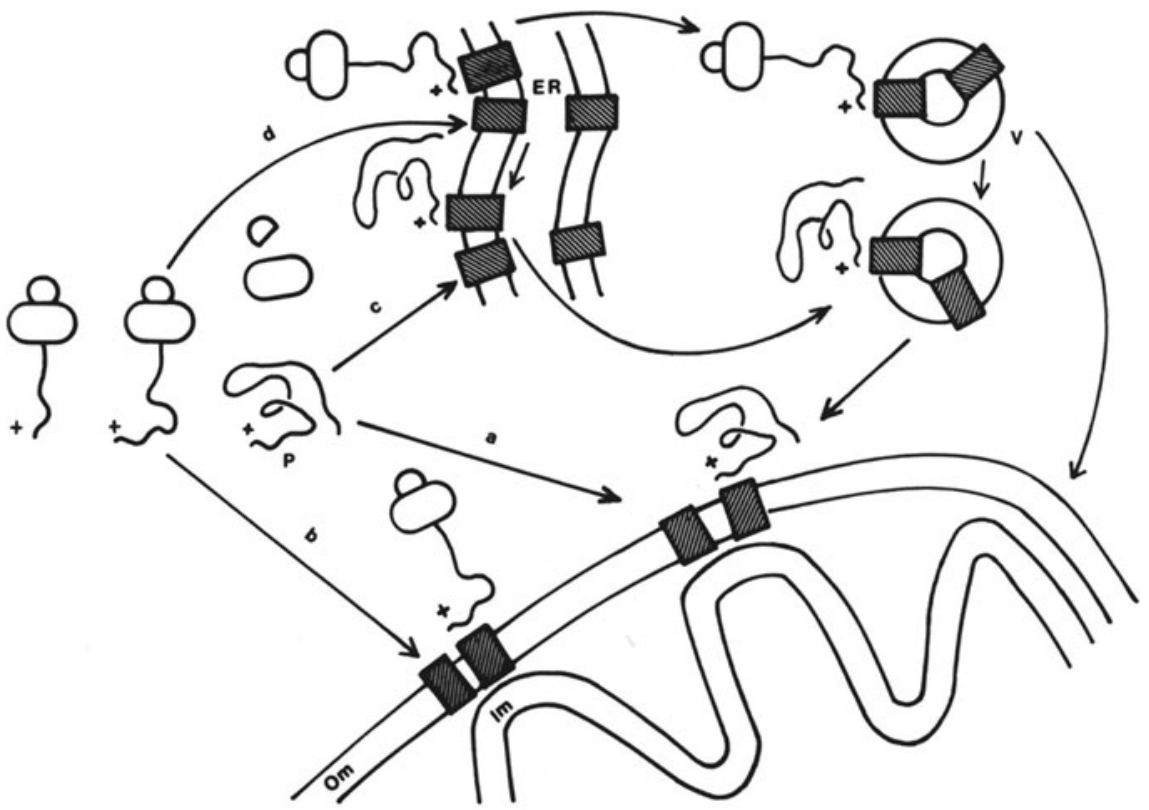

FIGURE 6. The figure is aimed at rationalizing observations on involvement of ribosomes bound not only to the outer membrane of terminal organelles, but also to the endoplasmic reticulum, in the genesis of some migratory proteins, here a mitochodrial protein. If the precursor of the protein, $\mathrm{P}$, is released in the cytoplasm, it binds post-translationally to the outer membrane receptor as shown in route a. The nascent precursor chain, however, can also bind to the outer membrane receptor before chain termination, that is, cotranslationally, route $b$; completion of the chain and import occur subsequently. Routes through the endøplasmic reticulum (ER) are also envisaged. The completed precursor binds to the receptor on the endoplasmic reticulum and reaches the outer membrane after passage through vesicles ( $v$ ) deriving from the endoplasmic reticulum, route $c$. Alternatively, the uncompleted chain can bind to the endoplasmic reticulum, route $d$, then the outer membrane through vesicles (v). As the translation continues, the uncompleted chain bound to the ER can be achieved before reaching transport vesicles. Completion of the chain can also occur on these vesicles. Binding of the precursor or uncompleted chains still linked to ribosomes to transport vesicles (not shown) and subsequent docking to the outer membrane are also possible. 
a mitochondrial protein. It rationalizes, in particular, the presence of ribosomes bound to the endoplasmic reticulum in the genesis of some migratory proteins and also explains cases of the observed involvement of ribosomes bound to the terminal organelles, as, for example, that of ribosomes bound to mitochondria (Ades and Butow, 1980a,b; Kellems et al., 1974, 1975; Schatz and Butow, 1983; Suissa and Schatz, 1982). In such cases, not only a cotranslational binding but also at least for some cases a cotranslational processing, qualified as a "pseudo-cotranslational event" (Etémadi, 1985) could occur. In a recent report, the cotranslational translocation of a precursor, common to two mitochondrial enzymes (acetylglutamate kinase and acetylglutamyl phosphate reductase) was not excluded (Wandinger and Weiss, 1987).

The proposal by Fujiki et al. (1984) and Suzuki et al. (1987) of the formation of peroxisomes from preexisting peroxisomes is not in essential contradiction with this hypothesis, if it is admitted that membrane material, lipids (Crane et al., 1983; Lord and Roberts, 1982), and some proteins (Lord and Roberts, 1982) can be brought by membrane vesicles deriving from the endoplasmic reticulum; these would thus constitute some sort of "pre-microbody" structures to be "matured" by further post-translational incorporation of matrix proteins and additional membrane proteins, and/or fuse with pre-existing peroxisomes. Provision of material from the endoplasmic reticulum together with post-translational supply of proteins might well lead to fission events in "proliferating" microbodies.

\subsection{The Case of Endoproteins}

"Endoproteins" were thus named in order to distinguish them from "ectoproteins" (Rothman and Lenard, 1977). Since ectoproteins are integral membrane proteins having an exposure at the side remote from the cytoplasm, either with or without an exposure at the cytosolic compartment, endoproteins were defined as integral membrane proteins with only a cytoplasmic exposure. Their membrane-embedded segment, which for the best representative of these proteins, the endoplasmic reticulum membrane protein, cytochrome $b_{5}$, is the $\mathrm{C}$ terminus of the molecule (see Daily and Strittmatter, 1981a,b; Enoch et al., 1979), is shown as penetrating only halfway through the hydrophobic core of the membrane (see Rothman and Lenard, 1977). Recently, Thiede et al. (1986) reported the case of the rat liver endoplasmic reticulum enzyme, stearoyl-CoA desaturase, which like cytochrome $b_{5}$ is synthesized on free ribosomes. The exact mode of membrane insertion of this protein, which exhibits $62 \%$ of the hydrophobic residues, has not been elucidated. Recent work by Strittmatter $e t$ al. (1988) shows, however, that at least $33 \mathrm{~N}$-terminal residues of the protein are not involved in membrane insertion. Another endoplasmic reticulum membrane protein, $\mathrm{NADH}$ cytochrome $b_{5}$ reductase, was thought to be C-terminally 
membrane anchored (Mihara et al., 1978). More recently, however, binding through the $\mathrm{N}$ terminus was admitted for this enzyme (Kensil and Strittmatter, 1986; Kensil et al., 1983). Furthermore, recent data suggest pentration of the $\mathrm{C}$ terminus of cytochrome $b_{5}$ deeply in the hydrophobic core, probably through the entire thickness of the membrane (Gogol and Engelman, 1984; Takagaki et al., 1983a,b). More recently Rzepecki et al. (1986) confirmed the deep penetration of C-terminal of cytochrome $b_{5}$ and Arinc et al. (1987) reported the external exposure of the extreme $\mathrm{C}$-terminal residues in tightly reconstituted cytochrome $b_{5}$.

Several experiments indicate that both cytochrome $b_{5}$ (see Okada et al., 1982; Rachubinski et al., 1980) and NADH cytochrome $b_{5}$ reductase (see Borgese and Gaetani, 1982; Okada et al., 1982) are synthesized on free ribosomes and post-translationally membrane inserted. This would also be the case for stearoyl-CoA desaturase (Thiede et al., 1986). These proteins thus furnish examples of integral membrane proteins bound post-translationally to the membrane, mostly to the endoplasmic reticulum but also to other membranes (for more references and details see Etémadi, 1985). Noteworthy is the fact that reconstitution by the direct insertion method leads only to a "loose" binding of cytochrome $b_{5}$ (Enoch et al., 1979; Takagaki et al., 1983a), small vesicles preferentially incorporating the protein (Greenhut and Roseman, 1985), while a "tight" binding, reminiscent of physiological insertion, does not require energy but the prior presence of a protein(s) or factors of membrane perturbation. The use of a detergent dialysis method (Enoch et al., 1979; Takagaki et al., $1983 b$ ) or incubation of cytochrome $b_{5}$ in detergent micelles with preformed vesicles (Christiansen and Carlsen, 1985), and more generally defects in membrane structure (Jain and Zakim, 1987; Scotto et al., 1987), lead to a tight binding.

Recent data indicate that some proteins of mitochondrial outer membrane and of the membrane of microbodies behave as endoproteins inasmuch as they are also synthesized on free ribosomes and inserted post-translationally into the organellar membrane, without energy requirement. A mitochondrial outer membrane protein, porin (Freitag et al., 1982a,b; Gasser and Schatz, 1983; Mihara and Blobel, 1982; Zwizinski et al., 1984), and some other proteins of this membrane of 14,000, 45,000, and 70,000 Da (Gasser and Schatz, 1983; Riezman, 1982) were reported to be post-translationally inserted in the outer membrane, without energy requirement. It has been proposed that all mitochondrial outer membrane proteins might be inserted post-translationally (Gasser and Schatz, 1983). Recently, a major peroxisomal membrane protein of 21,700 Da was also found to be synthesized predominantly on free ribosomes (Fujiki et al., 1984) and Suzuki et al. (1987) reported synthesis of three major peroxisomal membrane polypeptides of rat liver of 22,000, 26,000, and 70,000 
Da mostly on free polysomes. Such proteins are proposed to be synthesized without an extra-sequence (Fujiki et al., 1984; Koester et al., 1986; Suzuki et al., 1987). However, further work is necessary to better understand the genesis of outer membranes of organelles through which migratory proteins are translocated. Some observations reveal the presence of a common antigenic determinant between the outer mitochondrial membrane and rough microsomes or involvement of bound ribosomes in the genesis of some outer membrane proteins (Noel et al., 1985). Also, in a recent report Hartl and Just (1987) suggest that some drug-induced rat liver peroxisomal membrane proteins of different molecular weight $(42,000,28,000$, and $26,000 \mathrm{Da})$ derive by proteolysis from a common $69,000 \mathrm{Da}$ membrane protein; thus, the apparent multiplicity of membrane proteins synthesized on free ribosomes might in some cases find its origin in proteolytic cleavage of a single protein.

As to whether or not the previous presence of proteinaceous material (putative receptor(s)) is required for post-translational insertion of proteins in the outer membrane of organelles, contradictory reports exist. Thus, binding of porin was not affected by protease treatment of mitochondria (Gasser and Schatz, 1983), while other reports indicate inhibition of binding of this protein after mild proteolysis (Mihara and Blobel, 1982; Ono and Tuboi, 1987; Pfaller and Neupert, 1987; Zwizinski et al., 1984). As to whether porin can (Gasser and Schatz, 1983) or cannot (Mihara and Blobel, 1982; Ono and Tuboi, 1987) bind to the isolated outer mitochondrial membrane, an agreement is not reached. There are reports (Hase et al., 1986; Mihara and Sato, 1985) indicating the similarity of the $\mathrm{N}$ termini of porin and the 70,000 Da outer membrane protein. It is, in addition, considered that receptors for proteins inserted in the outer membrane also interact with migratory proteins that cross the outer membrane to reach intramitochondrial compartments (Hase et al., 1986; Hurt and Van Loon, 1986; Pfaller and Neupert, 1987). If so, binding of porin to trypsintreated mitochondria (Gasser and Schatz, 1983) would mean that either the proteinaceous material was at least not cleaved in a manner detrimental to the receptor function or, less likely, that protein receptors for many mitochondrial proteins do not exist. Clearly, the problem is strongly correlated with the very concept of the mechanism of migratory protein translocation. A recent report by Ono and Tuboi (1987) indicates that membrane potential is not required for insertion of porin which, it is suggested, occurs through the contact sites between the outer and the inner membrane before its definitive integration into the outer membrane. Studies by Kleene et al. (1987) reveal that the presence of nucleotide triphosphates is mandatory for insertion of porin and, more recently, Pfanner et al. (1988) proposed their involvement in the unfolding of porin. In fact their effect could be replaced by an acid treatment followed by a rapid alkali neutralization. 


\section{PROTEIN TRANSLOCATION THROUGH OR INSERTION INTO LIPID AGGREGATES}

\subsection{Experiments Related to Translocation of Secretory and Integral Membrane Proteins}

While abundant studies to date concerned various aspects of protein translocation process by reconstitution in its general sense, not many physiologically significant experiments of reconstitution using liposomes have been carried out, despite the potential aptitude of such experiments to solve some crucial problems. Reconstitution in the restricted sense is aimed essentially and most generally at mimicking the properties of membrane proteins by their insertion in lipid bilayers. Various procedures for such insertion were developed (see Etémadi, 1985; Racker, 1979). The possibility of a functional reconstitution by these procedures, and particularly through the direct insertion method in which proteins or complexes are allowed to insert into preformed liposomes, led to the hope that such a procedure might be relevant to physiological protein translocation processes. A series of work (for references see Wickner, 1979) on insertion of $E$. coli phage $\mathrm{M}_{13}$ coat protein led to the proposal that this protein is inserted with an absolute asymmetry in liposomes formed by the detergent dilution procedure and from this it was concluded that hydrophobic forces determine the orientation of integral membrane proteins in biological membranes. However, the insertion of the coat protein with an absolute asymmetry could not be substantiated by results reported by other authors (Chamberlain et al., 1978; $\mathrm{Hu}$ and Wiesnieski, 1979), while in the bacterial membrane the protein is asymmetrically oriented (Ohkawa and Webster, 1981). As detailed elsewhere (Etémadi, 1985), numerous examples of reconstitution clearly show that usually no unique natural orientation of membrane proteins is established by reconstitution procedures, such as detergent dilution or dialysis. Furthermore, a consensus transmembrane protein $\left(\mathrm{H}-2 \mathrm{~K}^{\mathrm{K}}\right)$ was reported to be inserted with a hairpin conformation and not in a transmembrane manner (Cardoza et al., 1984).

The "membrane trigger hypothesis" (Wickner, 1979) considered that membrane insertion of proteins occurs post-translationally without involvement of membrane receptor-translocators, or membrane-ribosome interaction, and that no energy is required for the process; the only thermodynamics of membrane-protein interaction would allow the correct insertion of precursors into the membrane. Post-translational insertion of precoat protein in preformed phospholipid vesicles into which signal peptidase, the enzyme cleaving the signal peptide of a series of precursors of exported proteins in E. coli membrane, was incorporated has been reported to occur with the $\mathrm{N}$ terminus of the protein in the luminal side (Ohno-Iwashita and Wickner, 1983; Watts et al., 1981). However, the role envisaged for the cleavage enzyme in the translocation pro- 
cess (Watts et al., 1981) was more recently denied (Geller and Wickner, 1985). Thus, we are left with the considerations of the membrane trigger hypothesis that the only protein-lipid interaction is at the basis of the physiological insertion of precoat protein. This, however (Rhoads et al., 1984), is contradictory to the report by Date et al. (1980), which states that membrane potential is required for transmembrane insertion of the same protein in vivo. The case of precoat protein, besides such contradictory proposals, cannot be taken as representative of most proteins translocated through or inserted into the membrane (for a discussion see Etémadi, 1985). Nevertheless, other attempts to reconstitute translocation of $E$. coli exported proteins into liposomes, into which the signal peptidase was inserted, were made. As a result, OmpA and maltosebinding protein were claimed to be correctly translocated and processed (Zimmermann and Wickner, 1983). Surprisingly, however, the processed proteins were found not to be membrane inserted for the case of OmpA, which is an outer membrane protein, or present in the luminal side in the case of maltosebinding protein, which is a soluble periplasmic protein, but released into the medium. The most rational interpretation would be that translocation did not occur but that some residual cleavage enzyme activity oriented toward the medium cleaved the precursors.

Some studies attempted to get insight into the interaction of signal sequences with membranes using model experiments in which synthetic signals were interacted with liposomes. In one such study (Nagaraj, 1984), the synthetic signal sequence for chicken lysozyme was labeled with the fluorescent dansyl(5-dimethyl-aminonaphtalene-1-sulfonyl) group and its interaction with phospholipid liposomes by fluorescence emission and polarization was examined. It was found that the sequence binds to the hydrophobic core of the phospholipid. In other studies, synthetic signals and their "mutated" counterparts were interacted with lipids and results were compared with the efficiency of such sequences when involved in genuine protein translocation processes. For example, Briggs and Gierasch (1984) prepared synthetic peptides with the sequence of the signal for the wild type or mutant (export defective) lambda phage receptors. A 7-residue-long segment of the wild-type signal flanked by a proline and a glycine residue (see Table I) is predicted to have an $\alpha$-helical conformation, while 4 of these residues are absent in export defective mutants. Using the circular dichroism of synthetic peptides in helix-promoting environments (trifluoroethanol or SDS), the relative helicities of the wild-type and defective signals were compared. While the wild-type signal showed CD spectra characteristic of a partial helical conformation, defective polypeptides did not show such spectra. When proline or glycine in defective signals was substituted by a leucine or cysteine, respectively-substitutions that regenerate the signal function - the predicted helix promotion could be confirmed by CD data. The highest helicity, however, was associated with the wild-type signal. In 
further studies, Briggs et al. (1985), using wild-type, defective, and revertant signal peptides, examined their interaction with a lipid monolayer at a waterair interface, their conformational change in the presence of phospholipid vesicles, and their aptitude to promote vesicle aggregation. Such studies led to the proposal that wild-type and pseudorevertant signals interact with lipids and that this aptitude correlates with the tendency of functional signals to form the $\alpha$ helical conformation. It was suggested that while the probability for interaction of signal sequences with membrane protein components exists, they may also have some contact with membrane lipid during secretion. The idea of an interaction with lipids was reinforced when it was observed (Briggs et al., 1986) by circular dichroism and Fourier transform infrared spectroscopy that the signal of the wild-type lambda phage receptor can be inserted in phospholipid monolayer and possesses an $\alpha$-helical structure, while the signal sequence in the aqueous phase is unstructured and interaction, without insertion, induces a $\beta$-structure. A model for interaction of the signal sequence with the membrane through involvement of SRP and docking protein, or other factors such as the membrane potential, is presented. However, only lipids would be involved in subsequent events (Figure 7). Nevertheless, recently, such a proposal was somewhat mitigated (Chen et al., 1987) when it was observed that the peptide corresponding to the functional signal as well as two peptides corresponding to pseudorevertant types of defective mutant were able to inhibit the in vitro translocation of the alkaline phosphatase and the outer membrane protein $\mathrm{A}$ in $E$. coli inverted inner membrane vesicles. The defective mutant peptide did not show such an effect. As a result, it was not decided whether the competition occurs at the level of a receptor in the membrane or with a cytoplasmic SRPlike material. In addition, an indirect inhibitory action by interaction with lipids was not excluded.

The above experiments thus concern the early step of the binding of nascent chains to the membrane. Other reports by Fidelio et al. $(1986,1987)$ consider the whole process of translocation. The authors examined surface properties of some proteins (pretrypsinogen, ovalbumin) and compared them to those of signal sequences (signal sequence of pretrypsinogen, residues 21-47 of ovalbumin constituting the putative uncleaved signal of this protein and a synthetic "consensus" signal sequence). It was found that while proteins present in the subphase interact with phospholipid monolayers at the air-water interface, signal sequences do not. The reason for discrepancy with the above results of Briggs et al. (1986) is not clear, despite the remark made that in those experiments a prokaryotic signal was used. In any event, the observation was interpreted to indicate the requirement for a "signal recognition particle" in binding the signal or involvement of receptor proteins in the microsomal membrane (Fidelio et al., 1986). The proposal of a mechanism for protein translocation stands on additional observations. The surface stability of a signal 

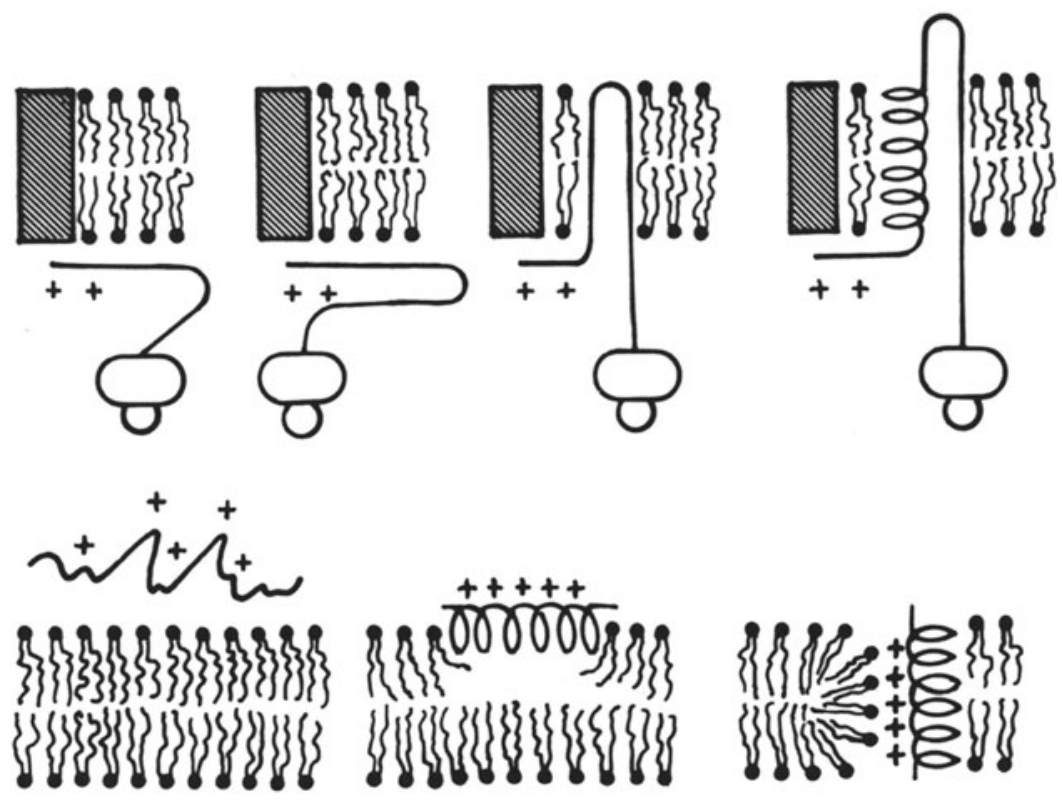

FIGURE 7. The upper drawing shows the suggested intraction of the nascent chain of a secretory or transmembrane integral protein with the membrane (Briggs et al., 1986). Proteinaceous material (hatched rectangles) or membrane potential might be involved in the initial targeting to the membrane. However, the subsequent interaction is with phospholipids only and leads from an unstructured conformation of the soluble state to the $\beta$-structure of the signal sequence. This structure is then presumably inserted into the membrane and gains the preferred $\alpha$-helical conformation. No protein is involved in the actual membrane insertion. The lower drawing concerns the interaction of the $\mathrm{N}$-terminal extrasequence of a mitochondrial migratory protein with the outer membrane (Roise et al., 1986; Von Heijne, 1986). It is presumed that ineraction with lipids in the cytoplasmic side (here the upper side of the bilayer) would lead to an amphipathic helical conformation of the extrasequence, which would subsequently be inserted in the membrane. Thus, proteinaceous receptors would not be involved in membrane insertion.

sequence when spread on the air-water interface alone or in mixed films with phospholipids was higher than that of proteins spread under similar conditions. Also, above a threshold of peptide or protein concentration, which was at the molar fraction of 0.2 for the signal and only of $0.0025-0.005$ (depending on the lipid used) for ovalbumin, lateral immiscibility appears as revealed by observation of two collapse points. Under these conditions the signal sequence also supported higher lateral surface pressure than albumin. Based on these observations, the following translocation mechanism was suggested. After the uncleaved signal sequence of a protein such as ovalbumin is guided into the 
lipid core of the membrane, the part corresponding to the mature chain would spontaneously enter into the membrane, the critical molar fraction of $0.0025-$ 0.005 would rapidly be attained, and a lipid rich and a protein-rich phase would separate. Protein extrusion from the membrane (translocation) would follow since the collapse pressure of the protein-rich phase is only about $22-24 \mathrm{mN} /$ $\mathrm{m}$, thus physical surface properties of signal sequences and protein chains would assist the understanding of the protein translocation, without necessity for a proteinaceous channel (Fidelio et al., 1987). However, in other studies (Robinson et al., 1987), involvement of a membrane protein in interaction with the signal sequence was recognized.

With regard to endoproteins, we have seen that experiment on reconstitution of cytochrome $b_{5}$ in lipid bilayers pointed to the requirement for the presence of protein or the presence or induction of membrane defects for an insertion apparently mimicking the natural membrane binding of the protein. In the case of $\mathrm{NADH}-$ cytochrome $b_{5}$ reductase attached through its $\mathrm{N}$ terminus to the endoplasmic reticulum membrane, the ultimate $\mathrm{N}$-terminal amino acid residue $\mathrm{Gly}_{1}$ is bound to a myristate moiety through an amide linkage. Kensil and Strittmatter (1986) found that after cholate solubilization and trypsinization, a 28 residue long $\mathrm{N}$-terminal segment remains bound to the cholate and can be reconstituted in phospholipid vesicles. Two possible conformations-a hairpin and a transmembrane one-were envisaged for this reconstituted segment through studies on the fluorescence of $\operatorname{Trp}_{16}$ and energy transfer from this residue to acceptors in the head group region of the outer monolayer. The hairpin conformation was preferred to the transmembrane conformation. The actual conformation of the $\mathrm{N}$ terminus of the enzyme in the microsomal membrane remains, however, unclear.

\subsection{Experiments on Translocation of Migratory Proteins}

Before the present understanding of the requirements of the post-translational translocation of migratory proteins (i.e., the possible interaction of signal sequences of precursors with membrane receptors and energy consumption), experiments on reconstitution of protein translocation used mature proteins. Assays carried out on interaction of cytochrome $c$ (holocytochrome $c$ ) with liposomal membranes are numerous (see Birrel and Griffith, 1976; Brown and Wütrich, 1977; Chapman and Urbina, 1971; Furuya et al., 1979; Mustonen et al., 1987). Generally, it is admitted that this interaction is electrostatic in nature, with phase separation and a long-range ordering effect on lipid. The occurrence in addition of a hydrophobic interaction was also admitted (Furuya et $a l .$, 1979). Such experiments, while eventually having a bearing on the interaction of cytochrome $c$ with the outer aspect of the inner mitochondrial membrane, do not concern the essential act of translocation through the outer mem- 
brane, which involves apocytochrome $c$ (see below) not holocytochrome $c$. Other experiments concerned the mature aspartate transaminase, which was found to react electrostatically with negatively charged lipid head groups. Furthermore, the occurrence of a hydrophobic interaction was recognized. Remarkable is the observation that in these experiments the enzyme was found to bind to vesicles in such a way that it was neither cleavable by protease action nor could it interact with its substrate (Furuya et al., 1979). Such observations indicate the degree of caution with which results of reconstitution experiment should be interpreted. The insensitivity to proteolysis was often taken as revealing protein translocation, while, on the other hand, a physiologically significant translocation involves the precursor, not the mature protein used in the present experiments. Also, observations on the interaction of the mature malate dehydrogenase and lipid vesicles and interpretation given are instructive. Under conditions in which the mature enzyme is present as a monomer (see Webster et al., 1979), a conformational change as a result of interaction with liposomes occurred. This was interpreted as reflecting a hydrophobic interaction of the protein with the membrane hydrophobic core and it was thought that these observations would have a bearing on protein translocation into mitochondria since it was supposed that matrix enzymes after similar iriteractions with the mitochondrial membrane would somehow reach the matrix compartment where they would be trapped by interaction with substrates, coenzymes, or the $\mathrm{pH}$ gradient. Here again, a whole theory reminiscent of the membrane trigger hypothesis was constructed, which involves interaction of the (mature) protein with membrane lipids without requirement of proteinaceous receptor-translocator system(s) or energy consumption.

Since the above early observations, most reconstitution experiments regarding the process of translocation of migratory proteins concerned either apocytochrome $c$ or only the initial steps of interaction of extrasequences with the membrane. Apocytochrome $c$ was found to bind to phospholipid vesicles containing acidic phospholipids. No complete translocation occurred, however, since all bound molecules could be digested by external proteases. Molecules were bound through their $\mathrm{C}$ termini to vesicles. This part of apocytochrome $c$ was protected from digestion. Remarkably, when proteases were entrapped in vesicles, most of the apocytochrome $c$ molecules were digested to small molecules. Thus, high-affinity receptors were thought not to be strictly required for translocation (Dumont and Richards, 1984). Other studies (for references see Rietveld et al., 1986; see also Walter et al., 1986) likewise indicated interaction of apocytochrome $c$ both electrostatically and through hydrophobic interaction with vesicles of negatively charged lipids. Interaction of apocytochrome $c$ with phosphatidylserine vesicles within which trypsin was entrapped led to its partial degradation, indicating that part of the molecule reached the luminal compartment. In vesicles of lipid mixtures containing phosphatidylcholine and phos- 
phatidylserine, the partial degradation of apocytochrome $c$ molecules occurred otpimally when vesicles were enriched to an abnormally high degree (50-100\%) in phosphatidylserine. A complete translocation did not occur, however. The exact part of the molecule reaching the luminal side is not clear. It was observed that as a result of cleavage from the inside no new binding sites were created for apocytochrome $c$, suggesting that the $\mathrm{C}$ terminus was not translocated (see Rietveld et al., 1986). This is the end of the molecule that is reported to bind apocytochrome $c$ to the mitochondrial membrane (Matsuura $e t$ al., 1981; Stuart et al., 1987). Thus, to date, a physiological translocation of apocytochrome $c$ is not reconstituted. Likewise the object of a recent report by Pilon et al (1987) was the definition of conditions allowing interaction with and insertion into lipids spread on monolayer surface of apocytochrome c. These assays using phosphatidylserine entities emphasize the importance of charge interaction. Another recent report by Park et al (1987), indicates, however, that the import of apocytochrome $\mathrm{c}$ by yeast mitochondria, when mostly methylated (as $\epsilon$ - $N$-trimethyllysine) - as it is naturally in this case-not only was not decreased but increased 2- to 4-fold. Thus, it would be interesting to assay reconstitution using the proteinanceous receptor and the enzyme-attaching heme to apocytochrome $c$ (Henning and Neupert, 1981; Henning et al., 1983; Zwizinski et al., 1984). The translocation of apocytochrome $c$ was in fact suggested to be a concerted process involving, besides the receptor, the apocytochromemodifying enzyme in some sort of group translocation event (Etémadi, 1985).

To get insight into the interaction of signal sequences of migratory proteins with the mitochondrial membrane, Ito et al. (1985) constructed synthetic peptides mimicking the structure of such sequences and assessed their effect on the import of cytochrome P-450 scc and adrenodoxin into adrenal cortex mitochondria. They proved to be generally inhibitory. However, binding of the precytochrome P-450 scc to the mitochondrial membrane was not affected. These peptides, in addition, uncoupled oxidative phosphorylation in isolated mitochondria from both rat liver and bovine adrenal cortex and at high concentrations led to the leakage of intermembrane and matrix mitochondrial enzymes, while membrane-bound enzymes were not solubilized. They were suggested to bind to the membrane, causing perturbations, possibly related to the function of extension sequences. It was proposed that "the function of the characteristic common sequences found in the extension peptide of mitochondrial enzyme precursors is not the specific recognition of mitochondrial surface, but the perturbation of the structure of mitochondrial membranes to enable the precursor peptide to translocate across the membrane." Gillepsie et al. (1985) synthesized a polypeptide corresponding to residues 1-27 of preornithine carbamyl transferase (see Table II) and also remarked that its interaction with mitochondria collapsed the membrane electrochemical potential. When this same peptide was added to a system (reticulocyte lysates) synthesizing the precursor of the 
enzyme, no collapse of electrochemical gradient occurred. Reasons for this discrepancy remain unclear. However, competition for a membrane receptor was admitted since a reversible inhibition of import occurred. Epand et al. (1986) used the same peptide and examined its interaction with various lipids. The peptide exhibited more $\alpha$-helical conformation in the presence of anionic lipids such as cardiolipin or dimyristoylphosphatidylglycerol. A zwitterionic phospholipid such as dimyristoylphosphatidylcholine did not affect the conformation of the peptide. The synthetic peptide disrupted large lipid aggregates and led to discoid micelles of $30-50 \mathrm{~nm}$ in diameter in which it was assumed to have an amphiphilic helix conformation. These observations led to the suggestion that bilayer perturbation might play a role during protein translocation into mitochondria. Roise et al. (1986) have synthesized three peptides of 15 , 25 , and 33 amino acids corresponding to the $\mathrm{N}$-terminal sequence of the precursor of subunit IV of yeast cytochrome $c$ oxidase. The second of these peptides has the sequence of the extrapeptide of 25 residues (see Table II). These peptides were all soluble in aqueous buffers. They could, however, be inserted into phospholipid monolayers at the water-air interface. The two largest peptides could disrupt phospholipid liposomes. A diffusion potential negative inside enhanced and a diffusion potential positive inside decreased this effect. These two peptides also uncoupled the respiratory control of isolated yeast mitochondria. In the presence of detergent micelles the 25-residue-long peptide became partially helical as revealed by circular dichroism measurements. In addition, the sequence of peptides allowed prediction of formation of amphiphilic helices, hence the suggestion that such helices play a crucial role in protein transport into mitochondria. A scheme was presented (Figure 7, lower drawing) in which the presequence of migratory proteins would insert as an amphiphilic helix in the membrane without involvement of receptor or any other proteinaceous material. The potential of most if not all the 23 mitochondrial targeting sequences to form amphiphilic helices was also recognized by Von Heijne (1986). The fact that synthetic extra-sequences exhibit membrane perturbing activity while precursors in which these sequences are linked to "passenger" proteins do not induce such an effect, was explained (Endo and Schatz, 1988) in considering that the passenger part would mask the amphiphilic properties of extra-sequences, which would appear by the unfolding of the protein in contact with the membrane. However, several considerations should be taken into account. As remarked by Maher and Singer (1986), it cannot be excluded that in such experiments and those mentioned above for synthetic signal sequences of secretory and membrane inserted proteins, interaction between peptides and membranes would not mimic that of precursor proteins during their translocation, since synthetic peptides might exist as oligomers, while natural translocation most probably involves interaction of individual entities. Furthermore, various synthetic presequences, bound $\mathrm{N}$-terminal to the 
mature part of a mitochondrial imported protein revealed the functionality of the synthetic prepeptides. These studies did not confirm the necessity for a potential amphiphilic $\alpha$-helical conformation for functional peptides (Allison and Schatz, 1986). On the other hand Skerjanc et al. (1987) could not confirm the implication of the membrane potential in binding of the synthetic pre-sequence of ornithine carbamyl transferase to lipid vesicles.

Aoyagi et al. (1987) have recently synthesized various peptides corresponding to different segments of the extra-sequence of cytochrome P-450 precursor which is of 39 residues. Two of these peptides representing residues 1-15 and 1-20 of the extra-sequence were active in inhibiting the import of some mitochondrial proteins, there were also peptides among those synthesized, that while exhibiting a random conformation in aquous buffer, showed a partially ordered conformation either $\alpha$-helical or type $\mathrm{II}^{\prime} \beta$ in the presence of buffer containing acidic liposomes [dipalmitoyl phosphatidylcholine plus dipalmitoyl phosphatidylglycerol $(3: 1)]$. The authors suggest that precursors change their conformation when they make contact with the surface of the inner membrane. However, the primary structure of the peptides could not allow an amphiphilic helix conformation. Thus, the authors propose that a partially ordered conformation such as $\alpha$-helix might be important, while amphiphilic $\alpha$-helix is not required. In addition the two active peptides caused leakage of acidic liposomes, which is interpreted to confirm that membrane perturbation may be important in mitochondrial import process. Assays of these peptides performed by Furuya et al. (1987) revealed that they did not affect the intactness of mitochondria as assessed by measurement of the respiratory control ratio. They decreased the rate of import of precursors while the binding of these to the mitochrondrial surface was not affected, meaning that a blockade of internalization was occurring. Thus, these results are discordant with proposals of other authors concerning inhibition of binding of precursors to mitochondria treated with polypeptides related to extra-sequences of mitochondrial imported proteins (see Gillespie et al., 1985; Yoshida et al., 1985). Recently Roise et al. (1988) were able to confirm that the potentiality for an amphiphilic $\alpha$-helix is not required for in vitro interaction of "artificial pre-sequences" with phospholipid monolayers or vesicles. The authors proposed, however, that such pre-sequences have to exhibit properties of amphiphilicity.

With regard to the crucial problem as to whether or not proteinaceous membrane components are implicated in binding of precursors through their extra-sequences, the significance of the above data is not clear. Again, Furuya et al. (1987) suggested a nonspecific interaction with lipids as evidenced by the fact that "active" peptides they synthesized could not only bind to mitochondria but also to microsomal membranes. However, it would remain to be clarified how such a nonspecific interaction would lead to specific organellar transport. In a recent study Myers et al. (1987) examined the interaction of the 
extra-sequence of ornithine carbamyl transferase with bilayer membranes of dipalmitoyl phosphatidylcholine containing small mole-percents of acidic phospholipids. A strong interaction with an enthalpy of association of $-60 \mathrm{kcal} /$ mol of peptide was observed. Fluorescence studies indicated, however, that the extra-sequence did not penetrate the bilayer. The authors, thus, do not exclude that further processing of precursor proteins would involve protein receptors and, finally, as discussed above, the involvement of membrane proteins, including an outer membrane protein, in the import of mitochondrial proteins was recognized (Ohba and Schatz, 1987).

Reconstitution experiments regarding chloroplast proteins also concerned the aspect of the initial binding of precursors (Pfisterer et al., 1982). Bitsch and Kloppstech (1986a,b) examined binding of such precursors to outer membrane vesicle preparations; after detergent treatment and reconstitution using sulfobetain or other detergents, the binding capacity was found in the residual particles after centrifugation and in the supernatant. Both a "partial reconstitution," that is, removal of detergent by dialysis of the whole detergent-treated vesicle preparations, and a "total reconstitution," defined as removal of detergent from the isolated supernatant, allowed an appreciable recovery of the binding activity.

\section{GENERAL AND CONCLUDING REMARKS}

In what precedes I have summarized the present state of the knowledge gained on physiological protein translocation processes by reconstitution experiments, reconstitution being taken in both its general and restricted senses. If the requirement for signal sequences interacting with specific receptor-translocators and the use of energy for translocation can be admitted, various processes of protein translocation with some exceptions such as that of endoproteins or apocytochrome $c$ can be said to follow the general mechanism of "some sort of active transport", previously suggested (Etémadi, 1980a, 1985) for migratory protein translocation.

Different authors impressed by the hydrophobic nature of signal sequences of secretory and transmembrane integral proteins proposed models for translocation in which only interaction with lipids was envisaged. Such an attitude, though appearing at first sight legitimate, does not correspond to the reality, which involves proteins. However, if the implication of proteins alone in interaction with signal sequences would ultimately prove to be true, it would remain to be explained how at the molecular level the interaction with the receptor occurs. Clarification of the point would presumably await a thorough knowledge of the nature of the receptor-translocator proteins. Also, for migratory proteins an initial interaction of extrasequences with lipids was envisaged. 
However, here again, as for secretory and transmembrane integral proteins, interaction with membrane proteins was ultimately admitted although much remains to be learned both on the initial steps of interaction and details of the process of translocation.

It is worthwhile at this point to mention a recent "unitary mechanism" for protein translocation processes presented by Singer et al. (1987a,b). This mechanism admits the presence of transmembrane translocator proteins. Each translocator protein is envisaged as a complex comprising $n(\sim 3-6)$ homologous but not identical subunits designated as $\alpha, \beta, \gamma, \delta$, which form a closed central aqueous channel (see Figure 8 ). Two classes of such translocator proteins, one for secretory and transmembrane integral proteins and the other for migratory proteins, would exist. Translocator proteins in addition to binding the signal sequence are involved in the formation of successive subdomains of

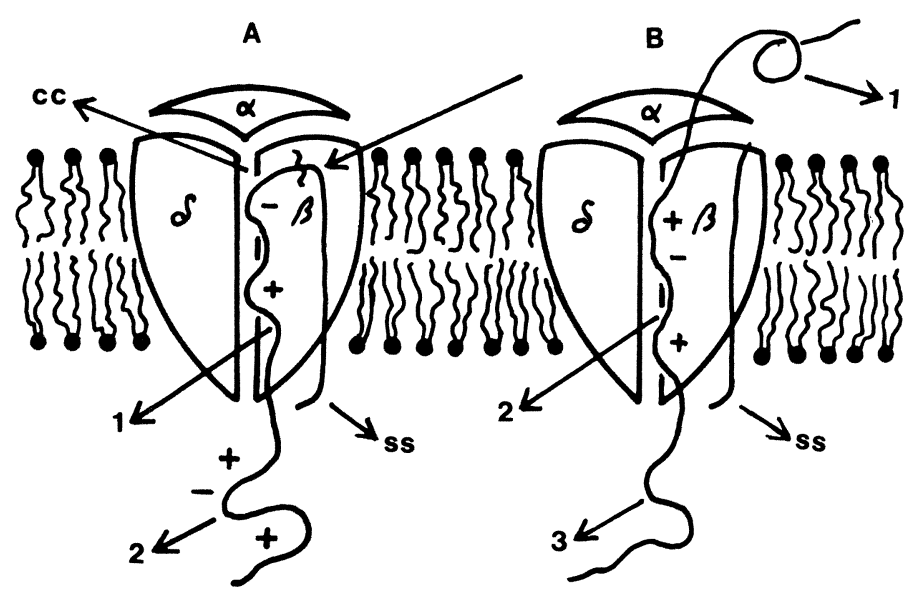

FIGURE 8. The unitary hypothesis for the mechanism of translocation of proteins (Singer et al., 1987a). The translocator protein is formed of subunits $\alpha, \beta, \gamma, \delta, \ldots$ One of the interfaces, where interaction between subunits is weaker, say the interface between $\beta$ and $\gamma$ subunits, is destined to allow translocation. Subunit $\gamma$ is not shown, so that the central aqueous channel (cc) appears. Translocation is suggested to be initiated by interaction of the signal sequence (ss) with $\beta$ or $\gamma$ subunit in such a way that its $\mathrm{N}$-terminal remains on the cis (cytoplasmic) side. The rest of the signal sequence interacts with the $\beta-\gamma$ interface, so that its site of cleavage (arrow in Figure $8 \mathrm{~A}$ ) reaches the trans (extracytoplasmic) face. Thus, necessarily, part of the mature chain enters the interface. As a result of interaction of the signal and the first segment of the mature chain with the interface, the central channel (cc) opens; subsequently, charged groups are located in contact with the aqueous environment of the channel, while hydrophobic residues face the hydrophobic core of the membrane. Translocation involves successive transfer of subdomains (indicated as 1 and 2 in Figure 8A and 1, 2, and 3 in Figure 8B). 
20-40 residues and in their energy-dependent translocation. Subdomains have secondary structures which could contain stretches of amphiphilic helix, or $\beta$ structure, or both. The first subdomain would be translocated as a result of an extensive quanternary rearrangement in translocator protein resulting from energy-dependent conformational changes in $\beta$ and/or $\gamma$ subunits. Translocation of the first subdomain pulls the following sequence into the interface where seeding of the second subdomain, followed by translocation, occurs. The process can thus continue until the whole protein is translocated. Translocation of secretory proteins without a cleaved signal is explained by the same mechanism except that the signal sequence, if it does not have too strong an affinity for the translocator protein, would form part of the last subdomain and be translocated. For a transmembrane protein with its $\mathrm{C}$ terminus located cytoplasmically, after the hydrophobic membrane anchoring segment is pulled in the interface, it slips laterally and forms a transmembrane helix; the $\beta-\gamma$ interface would close up, thus the hydrophobic segment acts as a stop transfer sequence. For proteins with their $\mathrm{N}$ termini located cytoplasmically and their $\mathrm{C}$ termini extracytoplasmically, it is admitted that the membrane-anchoring sequence is the uncleaved signal. For a protein with the $\mathrm{N}$ terminus located cytoplasmically but crossing the membrane thickness several times, the process of translocation begins as for proteins with uncleaved signals, then successive stop transfer and internal signals would be involved. At no stage is part of the protein sequence inserted directly into the lipid bilayer. A recent interesting report by Fikes and Bassford (1987) can be interpreted as supporting the idea that, depending on its affinity for the membrane, a signal sequence can anchor the protein in the membrane or be translocated with the mature part of the molecule. However, the problem of the chronology of the translocation of the uncleaved $\mathrm{N}$-terminal signal as compared to that of the bulk of the protein was not addressed in this work.

While the essence of the "unitary mechanism" for protein translocation presently seems acceptable and in accordance with the generalization of the involvement of "active transport" mechanisms (Etémadi, 1980a, 1985, 1987, 1988), the strict limitations placed as to the subunit structure of translocator proteins and the length of subdomains to be translocated would require substantiation. There are also aspects of the theory that would require caution. The orientation of the signal sequence restricted to the cytoplasmic exposure of its $\mathrm{N}$ terminus does not account for the presence of transmembrane proteins with extracytoplasmic location of their $\mathrm{N}$ termini (see Etémadi, 1986a) that do not have a cleaved extrasequence. Examples of such proteins are encountered both in eukaryotic and prokaryotic cells. Thus, bovine retinal opsin (Ovchinnikov et al., 1983; Schechter et al., 1979), rat cytochrome P-450 (Sakaguchi et al., 1987 ) and $E$. coli signal peptidase I (Moore and Miura, 1987) have their uncleaved membrane-anchored $\mathrm{N}$-terminal sequences oriented to the extracyto- 
plasmic side. In addition, the orientation of prebacteriorhodopsin in protoplasts of Halobacterium halobium is remarkable and does not comply with the proposal of Singer et al. (1987a,b). The protein normally has a cleaved extrasequence in the cells, which in protoplasts remains unprocessed and exposed on the trans side of the membrane (Seehara and Khorana, 1984). Remarkably, it was recently suggested (Von Heijne and Segrest, 1987) that the 13-residuelong extrasequence of the protein has the information to form an amphiphilic helix with a large nonpolar face and a narrow negatively charged polar face and that it would be inserted in the membrane contributing to one of the four helical hairpins. However, biological membranes being asymmetrical structures (see references in Etémadi, 1980a,b,c, 1985; Bergelson and Barsukov, 1978; Op Den Kamp, 1979; Van Deenen, 1981), only one orientation for the $\mathrm{N}$ terminus of membrane proteins and their precursors can be accepted. The proposal of Von Heijne and Segrest (1987) would require that the $\mathrm{N}$ terminus of the first hairpin be cytoplasmic, which is incompatible with the data of Seehara and Khorana (1984) on the localization of the N-terminal of the precursor of bacteriorhodopsin. However, if a reverted insertion of the first hairpin into the membrane is admitted-which, in passing, is not possible unless a concomitant insertion of a third hydrophobic segment is accepted - then both the cleavage site of the precursor and the $\mathrm{N}$-terminal of the membrane protein would be cytoplasmically located. Both these corollaries are contradicted, the first by the general localization of the active site of the cleavage enzyme that is extracytoplasmic and the second by the orientation of the $\mathrm{N}$ terminus of the mature protein in this case (Ovchinnikov, 1982). Thus, it would seem that neither a loop model nor a hair-pin model for insertion of the precursor of bacteriorhodopsin can account for the reality. With regard to migratory proteins, reservations as to the generality of the mode of insertion as proposed by the unitary hypothesis can also be expressed. Different authors (e.g., see Daum et al., 1982; Gasser et al., 1982b; Hurt and Van Loon, 1986; Kaput et al., 1982) suggested that the $\mathrm{N}$ termini of proteins of mitochondrial intermembrane space, inner membrane, and the matrix compartment are oriented extracytoplasmically. Schleyer and Neupert (1985) and Pfanner et al. (1987) have shown that the $\mathrm{N}$ termini of precursors of some migratory proteins reach the matrix and the extrasequence can be cleaved even under conditions in which part of the molecule is still cytoplasmic. A similar situation was also recently encountered during experiments on translocation of a hybrid protein to chloroplasts (see Kuntz et al., 1987). However, the above proposals and the reported observations can still be accommodated with the mechanism suggested by Singer et al. $(1987 a, b)$, since the localization of the cleaved sequence is not determined. In fact, in the above cases, it was not demonstrated that cleaved sequences were released in the mitochondrial matrix or the chloroplast stroma, so that a looplike model as suggested by Singer et al. (1987a) still has a chance to be appli- 
cable. Nonetheless, the recent demonstration by Hartl et al. (1987a,b) and Pfanner et al. (1987a) that, in the case of a mitochondrial intermembrane compartment protein, the entire precursor is first released in the matrix before being processed (see Figure 5a) cannot be explained on the basis of the suggested mechanism. Also, the path of translocation of ADP-ATP carrier protein (see Figure $5 b)$ does not comply with the unitary mechanism. With regard to chloroplast proteins, the uncleaved precursor of light harvesting chlorophyll a/b binding protein can also be imported, as reported by Chitnis et al. (1986), and even inserted in the thylakoid membrane, partly in an unprocessed precursor form. Chitnis et al. (1987) find in addition that this insertion requires a developmentally regulated stromal factor. Despite these reservations the mechanism proposed by Singer et al. $(1987 \mathrm{a}, \mathrm{b})$ confirms in essence the view that some sort of active transport is involved, in general, in protein translocation processes and strongly supports the statement made previously (Etémadi, 1985) that not a single case of physiologically significant reconstitution in its restricted sense was realized to date since in such experiments only interaction between the membrane lipids and translocated proteins was sought. In the light of our present knowledge, the minimal requirements formulated previously for a significant reconstitution of migratory proteins (Etémadi, 1985) can be extended to cases of secretory and transmembrane integral proteins. These requirements were emphasized to be the use of precursors of proteins, the translocation of which is examined, the prior reconstitution of receptor-translocators in the liposomal membrane in such a way that they exhibit at least in part a right-side orientation, provision of energy in an appropriate form, and soluble cofactors. In addition, it turns out that the proposal to use an in vitro translation system for reconstitution of translocation of secretory and transmembrane integral proteins remains valid, since such systems provide ATP used not only for translation but also for translocation (see in particular Chen and Tai, 1987b). In addition, recent reports regarding the feasibility of the in vitro post-translational translocation of such proteins in yeast (Hansen et al., 1986; Rothblatt and Meyer, 1986b; Waters and Blobel, 1986; Wiedmann et al., 1988) encourage designing in vitro translocation assays using completed precursors in order to determine the limits of such a process, which, as mentioned above, was questioned (Ibrahimi, 1987) for a case and which presumably should remain exceptional, at least under physiological in vivo conditions.

The generalized unitary mechanism of Singer et al. $(1987 \mathrm{a}, \mathrm{b})$ obviously does not account for translocation of endoproteins, of proteins like apocytochrome $c$, and of the proteins of the thylakoid membrane and of the luminal compartment of chloroplast thylakoids. It also dismisses the genesis and membrane insertion of transmembrane proteins crossing the membrane thickness once or several times and lacking cleaved $\mathrm{N}$-terminal sequences, but, bound to the membrane with their $\mathrm{N}$-termini located in the extracytoplasmic compartment. 
ACKNOWLEDGMENTS. I wish to thank my wife, Raymonde, for her indispensable assistance during the preparation of this manuscript. Financial support during this work was given by the Centre National de la Recherche Scientifique.

\section{REFERENCES}

Abrahamsen, L., Moks, T., Nilsson, B., Hellman, U., and Uhlen, M., 1985, Analysis of signals for secretion in the staphylococcal protein A gene. EMBO J. 4:3901-3906.

Adams, G. A., and Rose, J. K., 1985a, Structural requirements of a membrane spanning domain for protein anchoring and cell surface transport. Cell 41:1007-1015.

Adams, G. A., and Rose, J. K., 1985b, Incorporation of a charged amino acid into the membrane spanning region blocks cell surface transport but not membrane anchoring of a viral glycoprotein. Mol. Cell. Biol. 5:1442-1448.

Adelman, M. R., Sabatini, D. D., and Blobel, G., 1973, Ribosome-membrane insertion. Nondestructive disassembly of rat liver rough microsomes into ribosomal and microsomal components. J. Cell Biol. 56:206-229.

Ades, I. Z., and Butow, R. A., 1980a, The product of mitochondria-bound cytoplasmic polysomes in yeast. J. Biol. Chem. 255:9918-9924.

Ades, I. Z., and Butow, R. A., 1980b, The transport of proteins into yeast mitochondria, kinetics and pools. J. Biol. Chem. 255:9925-9935.

Adler, L. A., and Arvidson, S., 1987, Correlation between the rate of exoprotein synthesis and the amount of multiprotein complex or membrane-bound ribosome (MBRP-complex) in Staphylococcus aureus. J. Gen. Microbiol. 133:803-813.

Adrian, G. S., McCammon, M. T., Montgomory, D. L., and Douglas, M. G., 1986, Sequences required for delivery and localization of ADP/ATP translocator to the mitochondrial inner membrane, Mol. Cell. Biol. 6:626-634.

Ainger, K. J., and Meyer, D. I., 1986, Translocation of nascent secretory proteins across membranes can occur late in translation. EMBO J. 5:951-955.

Akiyama, Y., and Ito, K., 1985, The Sec Y membrane component of the bacterial protein export machinery: Analysis by new electrophoretic methods for integral membrane proteins. EMBO J. 4:3351-3356.

Akiyama, Y., and Ito, K., 1987, Topology of the Sec Y protein an integral membrane protein involved in protein export in Escherichia coli, EMBO J. 6:3465-3470.

Allison, D. S., and Schatz, G., 1986, Artificial mitochondrial presequences. Proc. Natl. Acad. Sci. U.S.A. 83:9011-9015.

Amar-Costesec, A., Todd, J. A., and Kreibich, G., 1984, Segregation of the polypeptide translocation apparatus to regions of the endoplasmic reticulum containing ribophorin and ribosomes. I. Functional tests on rat liver microsomal subfranctions. J. Cell Biol. 99:2247-2253.

Anderson, D. J., Walter, P., and Blobel, G., 1982, Signal recognition protein is required for the integration of acetylcholine receptor $\delta$ subunit, a transmembrane glycoprotein, into the endoplasmic reticulum. J. Cell Biol. 93:501-506.

Anderson, L., 1981, Identification of mitochondrial proteins and some of their precursors in twodimensional electrophoretic maps of human cells. Proc. Natl. Acad. Sci. U.S.A. 78:24072411.

Aoyagi, H., Lee, S., Kanmera, T., Mihara, H., and Kato, T., 1987, Interaction of synthetic fragments of the extension peptide of cytochrome P-450 (scc) precursor with phospholipid bilayer, J. Biochem. 102:813-820.

Arakawa, H., Takiguchi, M., Amaya, Y., Nagata, S., Hayashi, H., and Mori, M., 1987, cDNA- 
derived amino acid sequence of rat mitochondrial 3-oxoacyl-CoA thiolase with no transient presequence: Structural relationship with peroxisomal isoenzyme. EMBO J. 6:1361-1366.

Argan, C., and Shore, G. C., 1985, The precursor of ornithine carbamyl transferase is transported to mitochondria as a $5 \mathrm{~S}$ complex containing an import factor. Biochem. Biophys. Res. Commun. 131:289-298.

Argan, C., Lusty, C., and Shore, G., 1982, Nature of surface components on mitochondria which are required for import of ornithine carbamyl transferase. J. Cell Biol. 95(2, Pt. 2): 391a (Abstract).

Argan, C., Lusty, C. J., and Shore, G. C., 1983, Membrane and cytosolic components affecting transport of the precursor of ornithine carbamyl transferase into mitochondria. J. Biol. Chem. 258:6667-6670.

Arinc, E., Rzepecki, L. M., and Strittmatter, P., 1987, Topography of the C terminus of cytochrome $b_{5}$ tightly bound to dimyristoyl phosphatidylcholine vesicles, J. Biol. Chem. 262:1556315567.

Armstrong, J., Nieman, H., Smeekens, S., Rottier, P., and Warren, G., 1984, Sequence and topology of a model intracellular membrane protein $\mathrm{E}_{1}$ glycoprotin from coronavirus. Nature (London) 308:751-752.

Arpin, M., Matsuura, S., Margoliash, E., Sabatini, D. D., and Morimoto, T., 1980, A segment of cytochrome $c$ containing information for the uptake of newly synthesized cytochrome polypeptides by mitochondria. Eur. J. Cell Biol. 22:152 (Abstract M 451).

Audigier, Y., Friedlander, M., and Blobel, G., 1987, Multiple topogenic sequences in bovine opsin, Proc. Natl. Acad. Sci. USA 84:5783-5787.

Austen, B. M., 1979, Predicted secondary structures of amino-terminal extension sequences on secreted proteins. FEBS. Lett. 103:308-313.

Austen, B. M., and Ridd, D. H., 1983, Studies on the binding of a synthetic signal peptide to pancreatic rough microsomal vesicles. Biochem. Soc. Trans. 11:160-161.

Austen, B. M., Hermon-Taylor, J., Kaderbhai, M. A., and Ridd, D. H., 1984, Design and synthesis of a consensus signal sequence that inhibits protein translocation into rough microsomal vesicles. Biochem. J. 224:317-325.

Bacallao, R., Crooke, E., Shiba, K., Wickner, W., and Ito, K., 1986, The Sec Y protein can act post-translationally to promote bacterial protein export. J. Biol. Chem. 261:12907-12910.

Baker, A., and Schatz, G., 1987, Sequences from a prokaryotic genome on the mouse dihydrofolate reductase gene can restore the import of a truncated precursor protein into yeast mitochondria. Proc. Natl. Acad. Sci. U.S.A. 84:3117-3121.

Bakker, E. P., and Randall, L. L., 1984, The requirement for energy during export of $\beta$-lactamase in Escherichia coli is fulfilled by the total protonmotive force. EMBO J. 3:895-900.

Bancroft, C. F., Sussman-Berger, P., and Dobner, P. R., 1980, Biosynthesis of rat growth hormone and its messenger RNA. Ann. N.Y. Acad. Sci. 343:56-68.

Bankaitis, V. A., and Bassford, P. J. Jr., 1985a, Proper interaction between at least two components is required for efficient export of proteins to Escherichia coli cell envelope. J. Bacteriol. 161:169-178.

Bankaitis, V. A., and Bassford, P. J. Jr., 1985b, Sequences within the mature maltose-binding protein of Escherichia coli may be actively involved in initiating the export process. Ann. Inst. Pasteur Microbiol. 136B:3-7.

Bankaitis, V. A., Rasmussen, B. A., and Bassford, P. J. Jr., 1984, Intragenic suppressor mutations that restore export of maltose-binding protein with a truncated signal peptide. Cell 37:243252.

Bar-Nun, S., Kreibich, G., Adesnik, M., Alterman, L., Negishi, M., and Sabatini, D. D., 1980, Synthesis and insertion of cytochrome P-450 into endoplasmic reticulum. Proc. Natl. Acad. Sci. U.S.A. 77:965-969. 
Bassford, P. J. Jr., Silhavy, T. J., and Beckwith, J., 1979, Use of gene fusion to study secretion of maltose-binding protein in E. coli periplasm. J. Bacteriol. 139:19-31.

Bassuener, R., Wobus, U., and Rapoport, T. A., 1984, Signal recognition particle triggers the translocation of storage globulin polypeptides from field beans (Vicia faba L.) across mammalian endoplasmic reticulum membrane. FEBS Lett. 166:314-320.

Becker, W. M., Riezman, H., Weir, E. M., Titus, D. E., and Leaver, C. J., 1982, In vitro synthesis and compartmentation of glyoxysomal enzymes from cucumber. Ann. N.Y. Acad. Sci. 386:329-349.

Bedbrook, J. R., Smith, S. M., and Ellis, R. J., 1980, Molecular cloning and sequencing of cDNA encoding the precursor to the small subunit of chloroplast ribulose-1,5-bisphosphate carboxylase. Nature (London) 287:692-697.

Bedouelle, H., Bassford, P. J. Jr., Fowler, A. V., Zabin, I., Beckwith, J., and Hofnung, M., 1980 , Mutations which alter the function of the signal sequence of the maltose binding protein of Escherichia coli. Nature (London) 285:78-81.

Beevers, H., 1979, Microbodies in higher plants. Annu. Rev. Plant Physiol. 30:159-193.

Beevers, H., and Gonzalez, E., 1987, Proteins and phospholipids of glyoxysomal membranes from castor bean, Methods Enzymol. 148:528-532.

Behra, R., and Christen, P., 1986, In vitro import into mitochondria of the precursor of mitochondrial aspartate aminotransferase. J. Biol. Chem. 261:257-263.

Bell, G. I., Swain, W. F., Pictet, R., Cordell, B., Goodman, H. M., and Rutter, W. J., 1979, Nucleotide sequence of a cDNA clone encoding human preproinsulin. Nature (London) 282:525527.

Bergelson, L. D., and Barsukov, L. I., 1977, Topological asymmetry of phospholipids in membranes. The distribution of phospholipids in biological membranes is related to that in bilayer membranes of small vesicles. Science 179:224-230.

Bernstein, M., Hoffmann, W., Ammerer, G., and Schekman, R., 1985, Characterization of a gene product (Sec 53 p) required for protein assembly in yeast endoplasmic reticulum. J. Cell Biol. 101:2374-2382.

Bhat, N. K., and Avadhani, N. G., 1985, Transport of proteins into hepatic and nonhepatic mitochondria: Specificity of uptake and processing of precursor form of carbamyl phosphate synthetase. Biochemistry 24:8107-8113.

Bickman, E. R., Oliver, D. B., Garwin, J. L., Kumamoto, C., and Beckwith, J., 1984, The use of extragenic suppressors to define genes involved in protein export in Escherichia coli. Mol. Gen. Genet. 196:24-27.

Bielinska, R. M., Rogers, G., Rucinsky, T., and Boime, I., 1979, Processing in vitro of placental peptide hormones by smooth microsomes. Proc. Natl. Acad. Sci. U.S.A. 76:6152-6156.

Bingham, R. W., and Campbell, P. B., 1972, Studies on the biosynthesis of mitochondrial malate dehydrogenase and the location of its synthesis in liver cell of the rat. Biochem. J. 126:211215.

Birrel, G. B., and Griffith, O. H., 1976, Cytochrome $c$ induced lateral phase separation in a diphosphatylglycerol-steroid spin-label model membrane. Biochemistry 95:2925-2929.

Bitsch, A., and Kloppstech, K., 1986a, Reconstitution of the solubilized envelope receptors for nuclear-coded precursor proteins. Plant Biol. 2:241-246.

Bitsch, A., and Kloppstech, K., 1986b, Transport of proteins into chloroplasts. Reconstitution of the binding capacity for nuclear-coded precursor proteins after solubilization of envelope with detergents. Eur. J. Cell Biol. 40:160-166.

Blachly-Dyson, E., and Stevens, T. H., 1987, Yeast carboxypeptidase Y can be translocated and glycosylated without its aminoterminal signal sequence. J. Cell Biol. 104:1183-1191.

Black, S. D., French, J. S., William, C. H. Jr., and Coon, M. J., 1979, Role of hydrophobic 
polypeptide in the N-terminal region of NADPH-cytochrome P-450 reductase in complex formation with P-450 LM. Biochem. Biophys. Res. Commun. 91:1528-1535.

Blobel, G., 1980, Intracellular protein topogenesis. Proc. Natl. Acad. Sci. U.S.A. 77:1496-1500.

Blobel, G., and Dobberstein, B., 1975, Transfer of proteins across membranes. II. Reconstitution of functional rough microsomes from heterologous components. J. Cell Biol. 67:852-862.

Blobel, G., and Sabatini, D. D., 1971, Ribosome-membrane interaction in eukaryotic cells, in Biomembranes (L. A. Manson, ed.), Vol. 2, pp. 193-195, Plenum Press, N.Y.

Boeke, J. D., and Model, P., 1982, A prokaryotic membrane anchor sequence: Carboxyl terminus of bacteriophage $\mathrm{f}_{1}$ gene III protein retains it in the membrane. Proc. Natl. Acad. Sci. U.S.A. 79:5200-5204.

Boime, I., Szczesna, E., and Smith, D., 1977, Membrane dependent cleavage of the human placental lactogen precursor to its native form in ascites cell-free extract. Eur. J. Biochem. 73:515520.

Bonatti, S., and Blobel, G., 1979, Absence of a cleavable signal sequence in Sindbis virus glycoprotein $\mathrm{PE}_{2}$. J. Biol. Chem. 254:12261-12264.

Bonatti, S., Migliaccio, G., Blobel, G., and Walter, P., 1984, Role of signal recognition particle in the membrane assembly of Sindbis viral glycoproteins. Eur. J. Biochem. 140:499-502.

Borgese, N., and Gaetani, S., 1982, Biosynthesis and post-translational insertion into membranes of rat liver NADH-cytochrome $b_{5}$ reductase. J. Cell Biol. 95(2, Pt. 2):399a (abstract).

Bos, T. J., Davis, A. R., and Nayak, D. P., 1984, $\mathrm{NH}_{2}$ terminal hydrophobic region of influenza virus neuraminidase provides the signal function in translocation. Proc. Natl. Acad. Sci. U.S.A. 81:2327-2331.

Boutry, M., Nagy, F., Poulsen, C., Aoyagi, K., and Chua, N. H., 1987, Targeting of bacterial chloramphenicol acetyltransferase to mitochondria in transgenic plants. Nature (London) 328:340 342.

Bremer, E., Cole, S., Hindennach, I., Henning, U., Beck, E., Kurz, C., and Schaller, H., 1982, Export of a protein into the outer membrane of Escherichia coli. Stable incorporation of OmpA protein requires less than 193 amino terminal acid residues. J. Biochem. 122:223231.

Bretscher, M. S., 1973, Membrane structure: Some general principles. Science 181:622-629.

Briggs, M. S., and Gierasch, L. M., 1984, Exploring the conformational roles of sequences: Synthesis and conformational analysis of $\lambda$ receptor protein wild-type and mutant signal peptides. Biochemistry 23:3111-3114.

Briggs, M. S., Gierasch, L. M., Zlotnick, A., Lear, J. D., and De Grado, W. F., 1985, In vivo function and membrane binding properties are correlated for Escherichia coli Lam B signal peptides. Science 228:1096-1099.

Briggs, M. S., Cornell, D. G., Dluhy, R. A., and Gierasch, L. M., 1986, Conformation of signal peptides induced by lipids suggest initial steps in protein export. Science 233:206-208.

Brown, D. A., and Simoni, R. S., 1984, Biogenesis of 3-hydroxy-3-methyl glutaryl coenzyme A reductase, an integral glycoprotein of the endoplasmic reticulum. Proc. Natl. Acad. Sci. U.S.A. 81:1674-1678.

Brown, L. R., and Wütrich, K., 1977, NMR and ESR studies of the interactions of cytochrome $c$ with mixed cardiolipin-phosphatidylcholine vesicles. Biochim. Biophys. Acta 468:389-410.

Brown, P. A., Halvorson, H. O., Raney, P., and Perlman, D., 1984, Conformational alterations in the proximal portion of the yeast invertase signal peptide do not block secretion. Mol. Gen. Genet. 197:351-357.

Brusilow, W. S. A., Gunsalus, R. P., Hardeman, E. C., Decker, K. P., and Simoni, R. D., 1981, In vitro synthesis of $\mathrm{F}_{0}$ and $\mathrm{F}_{1}$ components of the proton translocating ATPase of Escherichia coli. J. Biol. Chem. 256:3141-3144. 
Büchel, D. E., Gronenbord, B., and Müller-Hill, B., 1980, Sequences of the lactose permease gene. Nature (London) 283:541-545.

Burns, D., and Lewin, A., 1986, Inhibition of the import of mitochondrial proteins by RNase. $J$. Biol. Chem. 261:6155-6163.

Burr, F. A., and Burr, B., 1981, In vitro uptake and processing of prezein and other maize proteins by maize membranes. J. Cell Biol. 90:427-434.

Burstein, V., and Schechter, I., 1978, Primary structure of N-terminal peptide segment linked to the variable and constant regions of immunoglobulin light chain precursors: Implication of the organization and controlled expression of immunoglobulin genes. Biochemistry 17:2392-2400.

Campbell, M. T., Sutton, R., and Pollak, J. K., 1982, Import of carbamoylphosphate synthetase into mitochondria from fetal rat liver. Eur. J. Biochem. 125:401-406.

Cancedda, R., and Schlesinger, M. J., 1974, Localization of polyribosomes containing alkaline phosphatase nascent polypeptides on membranes of Escherichia coli. J. Bacteriol. 117:290301.

Caplan, M. J., Palade, G. E., and Jamieson, J. D., 1986, Newly synthesized sodium-potassium ATPase $\alpha$-subunit has no cytosolic intermediate in MDCK cells. J. Biol. Chem. 261:2860 2865.

Cardoza, J. D., Kleinfeld, A. M., Stallcup, K. C., and Mescher, M. F., 1984, Hairpin configuration of $\mathrm{H}-2 \mathrm{~K}^{\mathrm{k}}$ in liposomes formed by detergent dialysis. Biochemistry 23:4401-4409.

Carlson, M., and Botstein, D., 1982, Two differently regulated mRNAs with different $5^{\prime}$ ends encode secreted and intracellular forms of yeast invertase. Cell 28:145-154.

Cascarano, J., Montisano, D. F., Pickett, C. B., and Jones, T. W., 1982, Rough endoplasmic reticulum-mitochondrial complexes from rat liver. Exp. Cell Res. 139:39-50.

Cashmore, A. R., 1983, Nuclear gene encoding the small subunit of ribulose-1,5-bisphosphate carboxylase, Basic Life Sci. 26:29-38.

Caulfield, M. P., Tai, P. C., and Davis, B. D., 1983, Association of penicillin-binding proteins and other enzymes with the ribosome-free membrane fraction of Bacillus subtilis. J. Bacteriol. 156: $1-5$.

Caulfield, M. P., Horiuchi, S., Tai, P. C., and Davis, B. D., 1984, The 64-kilodalton membrane protein of Bacillus subtilis is also present as a multicomplex on membrane free ribosomes. Proc. Natl. Acad. Sci. U.S.A. 81:7772-7776.

Caulfield, M. P., Furlong, D., Tai, P. C., and Davis, B. D., 1985, Secretory S complex of Bacillus subtilis forms a large organized structure when released from ribosomes. Proc. Natl. Acad. Sci. U.S.A. 82:4031-4035.

Caulfield, M. P., Duong, L. T., and Rosenblatt, M., 1986, Demonstration of post-translational secretion of human placental lactogen by a mammalian in vitro translation system. J. Biol. Chem. 261:10953-10956.

Chamberlain, B. K., Nozaki, Y., Tanford, C., and Webster, R. E., 1978, Association of the major coat protein of fd bacteriophage with phospholipid vesicles. Biochim. Biophys. Acta 510:1837.

Chapman, D., and Urbina, J., 1971, Phase transition and bilayer structure of Mycoplasma laidlawii B. FEBS Lett. 12:169-172.

Chen, L., and Tai, P. C., 1985, ATP is essential for protein translocation into Escherichia coli membrane vesicles. Proc. Natl. Acad. Sci. U.S.A. 82:4384-4388.

Chen, L., and Tai, P. C., 1987a, Effects of antibodies and other inhibitors on ATP-dependent protein translocation into membrane vesicles. J. Bacteriol. 169:2373-2379.

Chen, L., and Tai, P. C., 1987b, Evidence for the involvement of ATP in co-translational protein translocation. Nature (London) 328:164-166.

Chen, L., Rhoads, D., and Tai, P. C., 1985, Alkaline phosphatase and OmpA protein can be 
translocated post-translationally into membrane vesicles of Escherichia coli. J. Bacteriol. 161:973-980.

Chen, L., Tai, P. C., Briggs, M. S., and Gierasch, L. M., 1987, Protein translocation into Escherichia coli membrane vesicles is inhibited by functional synthetic signal peptides. J. Biol. Chem. 262:1427-1429.

Chen, W. J., and Douglas, M. G., 1987a, Phosphodiester bond cleavage outside mitochondria is required for the completion of protein import into mitochondrial matrix. Cell 49:651-658.

Chen, W. J., and Douglas, M. G., 1987b, The role of protein structure in the mitochondrial import pathway. Unfolding of mitochondrially bound precursors is required for membrane translocation, J. Biol. Chem. 262:15605-15609.

Cheng, M. Y., Pollock, R. A., Hendrick, J. A., and Horwich, A. L., 1987, Import and processing of human ornithine transcarbamylase precursor by mitochondria from Saccharomyces cerevisiae. Proc. Natl. Acad. Sci. U.S.A. 84:4063-4067.

Chia, C. P., and Arntzen, C. J., 1986, Evidence for two step processing of nuclear encoded chloroplast protein during membrane assembly. J. Cell Biol. 103:725-731.

Chien, S. M., and Freeman, K. B., 1983, Synthesis and uptake of precursor forms of mammalian mitochondrial proteins. Fed. Proc. 42:2125 (Abstract).

Chien, S. M., Patel, H. V., and Freeman, K. B., 1984, Import of rat liver mitochondrial malate dehydrogenase. Binding of the precursor to mitochondria, an intermediate stage in import. $J$. Biol. Chem. 259:13633-13636.

Chiocchia, K. B., and Dirckamer, K., 1984, Direct evidence for the transmembrane orientation of the hepatic glycoprotein receptors. J. Biol. Chem. 259:15440-15446.

Chitnis, P. R., Hartl, E., Kohorn, B. L., Tobin, E. M., and Thornber, J. P., 1986, Assembly of the precursor and processed light-harvesting chlorophyll $\mathrm{a} / \mathrm{b}$ protein of Lemna into the lightharvesting complex II of barley etioplasts, J. Cell. Biol. 102:982-988.

Chitnis, P., Nechushtai, R., and Thornber, J. P., 1987, Insertion of the precursor of the lightharvesting chlorophyll $\mathrm{a} / \mathrm{b}$ protein into the thylakoid requires the presence of a developmentally regulated stromal factor, Plant Mol. Biol. 10:3-11.

Christiansen, K., and Carlsen, J., 1985, Reconstitution of cytochrome $b_{5}$ into lipid vesicles in a form which is nonsusceptible to attack by carboxypeptidase Y. Biochim. Biophys. Acta 815:215222.

Chu, T. W., Grand, P. M., and Strauss, A. W., 1987a, Mutation of a neutral amino acid in the transit peptide of rat mitochondrial malate dehydrogenase abolishes binding and import, $J$. Biol. Chem. 262:15759-15764.

Chu, T. W., Grant, P. M., and Strauss, A. W., 1987b, The role of arginine residues in the rat mitochondrial malate dehydrogenase transit peptide, J. Biol. Chem. 262:12806-12811.

Chua, N. H., and Schmidt, G. W., 1978, Post-translational transport into intact chloroplasts of a precursor to the small subunit of ribulose 1,5-bisphosphate carboxylase. Proc. Natl. Acad. Sci. U. S.A. 75:6110-6114.

Chua, N. H., and Schmidt, G. W., 1979, Transport of proteins into mitochondria and chloroplasts. J. Cell Biol. 81:461-483.

Chyn, T. L., Martonosi, A. N., Morimoto, T., and Sabatini, D. D., 1979, In vitro synthesis of the $\mathrm{Ca}^{2+}$ transport ATPase by ribosomes bound to sarcoplasmic reticulum membranes. Proc. Natl. Acad. Sci. U.S.A. 76:1241-1245.

Claesson, L., Larhammer, D., Rask, L., and Peterson, P. A., 1983, cDNA clone for human invariant $\gamma$ chain of class II histocompatibility and its implications for protein structure. Proc. Natl. Acad. Sci. U.S.A. 80:7395-7399.

Cline, K., 1986, Import of proteins into chloroplasts. Membrane integration of a thylakoid precursor protein reconstituted in chloroplast lysates. J. Biol. Chem. 261:14804-14810. 
Cline, K., Werner-Washburne, M., Lubben, T. H., and Keegstra, K., 1985, Precursors to two nuclear-encoded chloroplast proteins bind to the outer envelope membrane before being imported into chloroplasts. J. Biol. Chem. 260:3691-3696.

Coleman, J., Inukai, M., and Inouye, M., 1985, Dual functions of signal peptide in protein transfer across the membrane. Cell 43:351-360.

Collier, D. N., Bankaitis, V. A., Weiss, J. B., and Bassford, P. J., Jr., 1988, The antifolding activity of Sec B promotes the export of $E$. coli maltose-binding protein, Cell 53:273-283.

Cornwell, K. L., and Keegstra, K., 1987, Evidence that a chloroplast surface protein is associated with a specific binding site for the precursor to the small subunit of ribulose 1,5-bisphosphate carboxylase, Plant. Physiol. 85:760-785.

Coruzzi, G., Brogli, R., Cashmore, A., and Chua, N. H., 1983, Nucleotide sequences of two pea cDNA clones encoding the small subunit of ribulose 1,5-bisphosphate carboxylase and the major chlorophyll a/b-binding thylakoid membrane polypeptide. J. Biol. Chem. 258:13991402.

Côté, C., and Boulet, D., 1985, Differential import and processing of the precursors to $F_{1}$-ATPase $\beta$-subunit and ornithine carbamyl transferase by liver, spleen, heart and kidney mitochondria. Biochem. Biophys. Res. Commun. 129:240-247.

Cover, W. H., Ryan, J. P., Bassford, P. J. Jr., Walsh, K. A., Bollinger, J., and Randall, L. L., 1987, Suppression of a signal sequence mutation by an amino acid substitution in the mature portion of the maltose-binding protein. J. Bacteriol. 169:1794-1800.

Crane, D., Holmes, R., and Masters, C. J., 1982a, Synthesis and incorporation of phospholipid by peroxisomes of mouse liver. Biochim. Biophys. Acta 712:57-64.

Crane, D. I., Holmes, R. S., and Masters, C. J., 1982b, Proteolytic modification of mouse liver catalase. Biochem. Biophys. Res. Commun. 104:1567-1572.

Crane, D. I., Holmes, R. S., and Masters, C. J., 1983, On the synthesis and incorporation of catalase and urate oxidase into the peroxisomes of mouse liver. Int. J. Biochem. 15:14291437.

Crimaudo, C., Hortsch, M., Gausephol, H., and Meyer, D. I., 1987, Human ribophorins I and II: the primary structure and membrane topology of two highly conserved rough endoplasmic reticulum-specific glycoproteins, EMBO J. 6:75-82.

Culter, D. F., and Garoff, H., 1986a, Mutants of the membrane binding region of Semliki forest virus $\mathrm{E}_{2}$ protein. I. Cell surface transport and fusogenic activity. J. Cell Biol. 102:889-901.

Culter, D. F., Melancon, P., and Garoff, H., 1986b, Mutants of membrane binding region of Semliki forest virus $\mathrm{E}_{2}$ protein. II. Topology and membrane binding. J. Cell Biol. 102:902910.

Dailey, H. A., and Strittmatter, P., 1981a, The role of $\mathrm{COOH}$-terminal anionic residues in binding cytochrome $b_{5}$ to phospholipid vesicles and biological membranes. J. Biol. Chem. 256:16771680.

Dailey, H. A., and Strittmatter, P. 1981b, Orientation of the carboxyl and $\mathrm{NH}_{2}$ termini of the membrane-binding segment of cytochrome $b_{5}$ on the same side of phospholipid bilayers. $J$. Biol. Chem. 256:3951-3955.

Dalbey, R. E., and Wickner, W., 1986, The role of the polar, carboxyl terminal domain of Escherichia coli leader peptidase in its translocation across the plasma membrane. J. Biol. Chem. 261:13844-13849.

Dalbey, R. E., and Wickner, W., 1988, Characterization of the internal signal anchor domain of Escherichia coli leader peptidase, J. Biol. Chem. 263:404-408.

Daniels, C. J., Bole, D. G., Quay, S. C., and Oxender, D. L., 1981, Role of membrane potential in the secretion of proteins into the periplasm of Escherichia coli. Proc. Natl. Acad. Sci. U.S.A. 78:5396-5400.

Date, T., Goodman, J. M., and Wickner, W. T., 1980, Procoat, the precursor of $\mathrm{M}_{13}$ coat protein, 
requires an electrochemical potential for membrane insertion. Proc. Natl. Acad. Sci. U.S.A. 77:4669-4673.

Daum, G., Gasser, S. M., and Schatz, G., 1982, Import of proteins into mitochondria. Energy dependent, two step processing of the intermembrane space enzyme cytochrome $b_{2}$ by isolated yeast mitochondria. J. Biol. Chem. 257:13075-13080.

Davis, B. D., and Tai, P. C., 1980, The mechanisms of protein secretion across membranes. Nature (London) 283:433-438.

Davis, N. G., and Model, P., 1985, An artificial anchor domain hydrophobicity suffices to stop transfer. Cell 41:607-614.

Davis, N. G., Boeke, J. D., and Model, P., 1985, Fine structure of membrane anchor domain. $J$. Mol. Biol. 181:111-121.

De Geus, P., Vehreij, H. M., Riegman, N. H., Hoekstra, W. P. M., and de Haas, G. P., 1984, The pro- and mature forms of the Escherichia coli K-12 outer membrane phospholipase A are identical. EMBO J. 3:1799-1802.

De Haan, M., Van Loon, A. P. G. M., Kreike, J., Vaessen, R. T. M. J., and Grivell, L. A., 1983, The biosynthesis of the ubiquinol-cytochrome $c$ reductase complex in yeast. DNA sequence analysis of the nuclear gene coding for the $14 \mathrm{kDa}$ subunit. Eur. J. Biochem. 138:169177.

De Lemos-Chiarandini, C., Frey, A. B., Sabatini, D. D., and Kreibich, G., 1987, Determination of membrane topology of the phenobarbital-induced cytochrome P-450 isoenzyme PB-4 using site specific antibodies, J. Cell. Biol. 104:209-219.

Della-Cioppa, G., Bauer, S. C., Klein, B. K., Shah, D. M., Fraley, R. T., and Kishore, G. M., 1986, Translocation of the precursor of 5-enoylpyruvoylshikimate-3-phosphate synthase into chloroplasts of higher plants in vitro. Proc. Natl. Acad. Sci. U.S.A. 83:6873-6877.

Della-Cioppa, G., Bauer, S. C., Taylor, M. L., Rochester, D. E., Klein, B. K., Shah, D. M., Fraley, R. T. and Kishore, G. M., 1987, Targeting a herbicide-resistant enzyme from Escherichia coli to chloroplasts of higher plants. Bio/Technology 5:579-584.

Desel, H., Zimmermann, R., Janes, M., Miller, F., and Neupert, W., 1982, Biosynthesis of glyoxysomal enzymes in Neurospora crassa. Ann. N.Y. Acad. Sci. 386:377-388.

Deshaies, R. J., and Schekman, R., 1987, A yeast mutant defective at an early stage in import of secretory protein precursors into the endoplasmic reticulum, J. Cell. Biol. 105:633-645.

Devillers-Thiery, A., Kindt, T., Scheele, G., and Blobel, G., 1975, Homology in amino-terminal sequence of precursors to pancreatic secretory proteins. Proc. Natl. Acad. Sci. U.S.A. 72:50165020.

DiRienzo, J. M., and Inouye, M., 1979, Lipid fluidity dependent biosynthesis and assembly of outer membrane proteins of E. coli. Cell 17:155-161.

DiRienzo, J. M., Nakamura, K., and Inouye, M., 1978, The membrane proteins of gram negative bacteria: Biosynthesis assembly and function. Annu. Rev. Biochem. 47:481-552.

Distel, B., Veenhuis, M., and Tabak, H. F., 1987, Import of alcohol oxidase into peroxisomes of Saccharomyces cerevisiae, EMBO J. 6:3111-3116.

Dorbani, L., Janczic, V., Linden, M., Leterrier, J. F., Nelson, B. D., and Rendon, A., 1987, Subfractionation of the outer membrane of rat brain mitochondria: Evidence for the existence of a domain containing the porin hexokinase complex. Arch. Biochem. Biophys. 252:188196.

Douglas, M. G., Geller, B. L., and Emr, S. D., 1984, Intracellular targeting and import of an $\mathrm{F}_{1}$ ATPase-subunit- $\beta$-galactosidase hybrid protein into yeast mitochondria, Proc. Natl. Acad. Sci. U.S.A. 81:3983-3987.

Douma, A. C., Veenhuis, M., Sulter, G. J., and Harder, W., 1987, A proton translocating adenosine triphosphatase is associated with the peroxisomal membrane of yeasts. Arch. Microbiol. 147:42-47. 
Dumont, M. E., and Richards, F. M., 1984, Insertion of apocytochrome $c$ into lipid vesicles. $J$. Biol. Chem. 259:4147-4156.

Duong, L. T., Caulfield, M. P., and Rosenblatt, M., 1987, Synthetic signal peptide and analogs display different activities in mammalian and plant in vitro secretion synthesis. J. Biol. Chem. 262:6328-6333.

Ehring, R., Beyreuther, K., Wright, J. K., and Overath, P., 1980, In vitro and in vivo products of $E$. coli lactose permease gene are identical. Nature (London) 283:537-540.

Eilers, M., Hwang, S., and Schatz, G., 1988, Unfolding and refolding of a purified precursor protein during import into isolated mitochondria, EMBO J. 7:1139-1145.

Eilers, M., and Schatz, G., 1986, Binding of a specific ligand inhibits import of a purified precursor protein into mitochondria. Nature (London) 382:228-232.

Eilers, M., Opplinger, W., and Schatz, G., 1987, Both ATP and energized inner membrane are required to import a purified precursor protein into mitochondria. EMBO J. 6:1073-1077.

Emr, S. D., and Bassford, P. J. Jr., 1982, Localization and processing of outer membrane and periplasmic proteins in Escherichia coli strains harboring export specific suppressor mutations. J. Biol. Chem. 257:5852-5860.

Emr, S. D., and Silhavy, T. J., 1982, Molecular components of the signal sequence that function in the initiation of protein export. J. Cell Biol. 95:689-696.

Emr, S. D., and Silhavy, T. J., 1983, Importance of secondary structure in the signal sequence for protein secretion. Proc. Natl. Acad. Sci. U.S.A. 80:4599-4603.

Emr, S. D., Hall, M. N., and Silhavy, T. J., 1980, Mechanism of protein localization: The signal hypothesis and bacteria. J. Cell Biol. 86:701-711.

Emr, S. D., Hanley-Way, S., and Silhavy, T. J., 1981, Suppressor mutations which restore export of a protein with a defective signal sequence. Cell 23:79-88.

Emr, S., Vassarotti, A., Garret, J., Geller, B., Takeda, M., and Douglas, M., 1986, The amino terminus of the yeast $\mathrm{F}_{1}$-ATPase $\beta$-subunit precursor functions as a mitochondrial import signal. J. Cell Biol. 102:523-533.

Endo, T., and Schatz, G., 1988, Latent membrane perturbation activity of a mitochondrial precursor protein is exposed by unfolding, EMBO J. 7:1153-1158.

Enequist, H. G., Hirst, T. R., Harayama, S., Hardy, S. J. S., and Randall, L. L., 1981, Energy is required for maturation of exported proteins in Escherichia coli. Eur. J. Biochem. 116:227233.

Engelman, D. M., and Steitz, T. A., 1981, The spontaneous insertion of proteins into and across membranes; the helical hairpin hypothesis. Cell 23:411-422.

Enoch, H. G., Fleming, P. J., and Strittmatter, P., 1979, The binding of cytochrome $b_{5}$ to phospholipid vesicles and biological membranes. Effect of orientation on intermembrane transfer and digestion by carboxypeptidase Y. J. Biol. Chem. 254:6483-6488.

Enosawa, S., and Ohashi, A., 1986, Localization of enzyme for heme attachment to apocytochrome $c$ in yeast mitochondria. Biochem. Biophys. Res. Commun. 141:1145-1150.

Epand, R. M., Hui, S. M., Argan, C., Gillespie, L. L., and Shore, G. C., 1986, Structural analysis and amphiphilic properties of a chemically synthesized mitochondrial signal peptide. J. Biol. Chem. 261:10017-10020.

Erickson, A. H., Walter, P., and Blobel, G., 1983, Translocation of a lysosomal enzyme across the microsomal membrane requires signal recognition particles. Biochem. Biophys. Res. Commun. 115:275-280.

Eskridge, E. M., and Shields, D., 1982, Cell-free processing of small polypeptide hormones is a co-translational event. J. Cell Biol. 95:394a (Abstract).

Etémadi, A.-H., 1980a, Membrane asymmetry. A survey and critical appraisal of the methodology. I. Methods for assessing the asymmetric orientation and distribution of proteins. Biochim. Biophys. Acta 604:347-422. 
Etémadi, A.-H., 1980b, Membrane asymmetry. A survey and critical appraisal of the methodology. II. Methods for assessing the unequal distribution of lipids. Biochim. Biophys. Acta 604:423-475.

Etémadi, A.-H., 1980c, Tendances organisationnelles des constituants des membranes biologiques et les problèmes de l'asymétrie de leur distribution. Biochimie 62:111-134.

Etémadi, A.-H., 1985, Functional and orientational features of protein molecules in reconstituted lipid membranes. Adv. Lipid Res. 21:281-428.

Etémadi, A.-H., 1986a, Mechanism of biogenesis and translocation of cell surface membrane proteins, in Handbook of Plasma Membranes, CRC Press, Boca Raton, FL.

Etémadi, A.-H., 1986b, Intracellular path and maturation of cell surface proteins, in Handbook of Plasma Membranes, CRC Press, Boca Raton, FL.

Etémadi, A.-H., 1987, Reconstitution and cellular protein translocation processes, in: International Symposium Membranes Lipids, Metabolism Organization, Varna, Abstract lecture 1-5.

Etémadi, A.-H., 1988, Overview on protein translocation and membrane insertion processes and conditions for their physiologically significant reconstitution, in: International Conference on Biomembranes in Health and Disease, Lucknow, Abstract Lecture 43.

Evans, E. A., Gilmore, R., and Blobel, G., 1986, Purification of microsomal signal peptidase as a complex. Proc. Natl. Acad. Sci. U.S.A. 83:581-585.

Fecycz, I. T., and Blobel, G., 1987, Soluble factors stimulating secretory protein translocation in bacteria and yeast can substitute for each other. Proc. Natl. Acad. Sci. U.S.A. 84:3723-3727.

Fenton, W. A., Hack, A. M., Helfgott, D., and Rosenberg, L. E., 1984, Biogenesis of mitochondrial enzyme methylmalonyl CoA mutase. Synthesis and processing of a precursor in a cellfree system and in cultured cells. J. Biol. Chem. 259:6616-6621.

Ferro-Novick, S., Novick, P., Field, C., and Schekman, R., 1984a, Yeast secretory mutants that block formation of active cell surface enzymes. J. Cell Biol. 98:35-43.

Ferro-Novick, S., Hausen, W., Sehaver, I., and Schekman, R., 1984b, Genes required for completion of import of proteins into the endoplasmic reticulum in yeast. J. Cell Biol. 98:44-53.

Fidelio, G. D., Austen, B. M., Chapman, D., and Lucy, J. A., 1986, Properties of signal sequence peptides at an air-water interface. Biochem. J. 238:301-304.

Fidelio, G. D., Austen, B. M., and Chapman, D., and Lucy, J. A., 1987, Interaction of ovalbumin and its putative signal sequence with phospholipid monolayers. Possible importance of differing lateral stabilities in protein translocation. Biochem. J. 244:295-301.

Fields, S., Winter, G., and Brownlee, G. G., 1981, Structure of neuraminidase gene in human influenza virus A/PR/8/34. Nature (London) 230:213-217.

Fikes, J. D., Bankaitis, V. A., Ryan, J. P., and Bassford, P. J., Jr., 1987, Mutational alterations affecting the export competence of a truncated but fully functional maltose-binding protein signal peptide, J. Bacteriol. 169:2345-2351.

Fikes, J. D., and Bassford, P. J. Jr., 1987, Export of unprocessed precursor maltose-binding protein to the periplasm of Escherichia coli cells. J. Bacteriol. 169:2352-2359.

Finidori, J., Rizzolo, L., Gonzalez, A., Kreibich, G., Adesnik, M., and Sabatini, D. D., 1987, The influenza hemagglutinin insertion signal is not cleaved and does not halt translocation when presented to the endoplasmic reticulum membrane as part of a translocating polypeptide. J. Cell Biol. 104:1705-1714.

Firguira, F. A., Hendrick, J. P., Kalousek, F., Kraus, J. P., and Rosenberg, L. E., 1984, RNA required for import of precursor proteins into mitochondria. Science 226:1319-1322.

Fitts, R., Reuveny, Z., Van Amsterdam, J., Mulholland, J., and Botstein, D., 1987, Substitution of tyrosine for either cysteine in $\beta$-lactamase prevents release from the membrane during secretion, Proc. Natl. Acad. Sci. USA 84:8540-8543.

Fluegg, V. I., and Hinz, G., 1986, Energy dependence of protein translocation into chloroplasts. Eur. J. Biochem. 160:563-570. 
Freitag, H., Janes, M., and Neupert, W., 1982a, Biosynthesis of mitochondrial porin and insertion into the outer mitochondrial membrane of Neurospora crassa. Eur. J. Biochem. 126:197202.

Freitag, H., Neupert, W., and Benz, R., 1982b, Purification and characterization of a pore protein of the outer mitochondrial membrane from Neurospora crassa. Eur. J. Biochem. 123:629639.

Freudl, R., Braun, G., Hindennach, I., and Henning, U., 1985, Lethal mutations in the structural gene of an outer membrane protein (OmpA) of Escherichia coli K-12. Mol. Gen. Genet. 210:76-81.

Freudl, R., MacIntyre, S., Degen, M., and Henning, U., 1988, Alterations to the signal peptide of an outer membrane protein (OmpA) of Escherichia coli $\mathrm{K}_{12}$ can promote either the cotranslational or the post-translational mode of processing, J. Biol. Chem. 262:344-349.

Freudl, R., Schwartz, H., Stierhof, Y. D., Gamon, K., Hindennach, I., and Henning, U., 1986, An outer membrane protein (OmpA) of Escherichia coli $\mathrm{K}-12$ undergoes a conformational change during export. J. Biol. Chem. 261:11355-11361.

Friedlander, M., and Blobel, G., 1985, Bovine opsin has more than one signal sequence, Nature 318:338-343.

Fujiki, Y., and Lazarow, P. B., 1982, Post-translational uptake of an in vitro synthesized peroxisomal polypeptide of rat liver. J. Cell Biol. 95(2, Pt. 2): 398a (Abstract).

Fujiki, Y., and Lazarow, P. B., 1985, Post-translational import of fatty acyl CoA oxidase and catalase into peroxisomes. J. Biol. Chem. 260:5603-5609.

Fujiki, Y., Rachubinski, R. A., and Lazarow, P. B., 1984, Synthesis of a major integral membrane polypeptide of rat liver peroxisomes on free polysomes. Proc. Natl. Acad. Sci. U.S.A. 81:7127-7131.

Furuta, S., Hashimoto, T., Miura, S., Mori, M., and Tatibana, M., 1982, Cell free synthesis of enzymes of peroxisomal $\beta$-oxidation. Biochem. Biophys. Res. Commun. 105:639-646.

Furuya, S., Okada, M., Ito, A., Aoyagi, H., Kanmera, T., Kato, T., Sagara, Y., Horiuchi, T., and Omura, T., 1987, Synthetic partial extension peptides of P-450 (scc) and adrenodoxin precursors: effects on the import of mitochondrial enzyme precursors, J. Biochem. (Tokyo) 102:821-832.

Furuya, E., Yoshida, Y., and Tagawa, K., 1979, Interaction of aspartate aminotransferase with negatively charged lecithin liposomes. J. Biochem. (Tokyo) 85:1157-1163.

Garcia, P. D., and Ghrayeb, J., Inouye, M., and Walter, P., 1987, Wild type and mutant signal peptide of Escherichia coli outer membrane lipoprotein interact with equal efficiency with mammalian signal recognition particle. J. Biol. Chem. 262:9463-9468.

Gardel, C., Benson, S., Hunt, J., Michaelis, S., and Beckwith, J., 1987, sec D, a new gene involved in protein export in Escherichia coli, J. Bacteriol. 169:1285-1290.

Garnier, J., Gaye, P., Mercier, J. C., and Robson, B., 1980, Structural properties of signal peptides and their membrane insertion. Biochimie 62:231-239.

Garwin, J. L., and Beckwith, J., 1982, Secretion and processing of ribulose-binding protein in Escherichia coli. J. Bacteriol. 149:789-792.

Gasser, S. M., and Schatz, G., 1983, Import of proteins into mitochondria. In vitro, studies on the biogenesis of the outer membrane. J. Biol. Chem. 258:3427-3430.

Gasser, S. M., Daum, G., and Schatz, G., 1982a, Import of proteins into mitochondria. Energy dependent uptake of precursors by isolated mitochondria. J. Biol. Chem. 257:13034-13041.

Gasser, S. M., Ohashi, A., Daum, G., Böhni, P. C., Gibson, J., Reid, G. A., Yonetani, T., and Schatz, G., 1982b, Imported mitochondrial proteins cytochrome $b_{2}$ and cytochrome $c_{1}$ are processed in two steps. Proc. Natl. Acad. Sci. U.S.A. 79:267-271.

Gearing, D. P., and Nagley, P., 1986, Yeast mitochondrial ATPase subunit 8, normally a mito- 
chondrial gene product, expressed in vitro and imported back into the organelle. EMBO J. 5:3651-3655.

Geering, K., Meyer, D., Paccolat, M. P., Kraehenbuhl, J. P., and Rossier, B. C., 1985, Membrane insertion of $\alpha$ - and $\beta$-subunits of $\mathrm{Na}^{+}, \mathrm{K}^{+}$-ATPase. J. Biol. Chem. 260:5154-5160.

Geller, B. L., and Wickner, W., 1985, $\mathrm{M}_{13}$ procoat inserts into liposomes in absence of other membrane proteins. J. Biol. Chem. 260:13281-13285.

Geller, B. L., Movva, N. R., and Wickner, W., 1986, Both ATP and the electrochemical potential are required for optimal assembly of proOmpA into Escherichia coli inner membrane vesicles. Proc. Natl. Acad. Sci. U.S.A. 83:4219-4222.

Geredes, H. H., Behrends, W., and Kindl, H., 1982, Biosynthesis of microbody matrix enzyme in greening cotyledons. Glycolate oxidase synthesized in vivo and in vitro. Planta 156:572578.

Gething, M. J., and Sambrook, J., 1982, Construction of influenza hemagglutinin genes that code for intracellular and secreted forms of the protein. Nature (London) 300:593-603.

Ghersa, P., Huber, P., Semenza, G., and Wacker, H., 1986, Cell free synthesis, membrane integration and glycosylation of pro-sucrase-isomaltase. J. Biol. Chem. 261:7969-7974.

Ghosh, M. K., and Hajra, A. K., 1986, Subcellular distribution and properties of acyl/alkyl dihydroxy acetone phosphate reductase in rodent livers. Arch. Biochem. Biophys. 245:523-530.

Ghrayeb, J., and Inouye, M., 1984, Nine amino acid residues at the amino terminal of lipoprotein are sufficient for its modification, processing and localization in the outer membrane of Escherichia coli. J. Biol. Chem. 259:463-467.

Ghrayeb, J., Lunn, C. A., Inouye, S., and Inouye, M., 1985, An alternate pathway for the processing of the prolipoprotein signal peptide in Escherichia coli. J. Biol. Chem. 260:1096110965.

Giam, C. Z., Chai, T., Hayashi, S., and Wu, H. C., 1984, Prolipoprotein modification and processing in Escherichia coli. A unique secondary structure in prolipoproteins signal sequence for the recognition by glyceryltransferase. Eur. J. Biochem. 141:331-337.

Gibbs, S. P., 1979, The route of entry of cytoplasmically, synthesized proteins into chloroplasts of algae possessing chloroplast ER. J. Cell Sci. 34:253-266.

Gietl, C., and Hock, B., 1984, Import of in vitro synthesized glyoxysomal malate dehydrogenase into isolated watermelon glyoxysomes. Planta 162:261-267.

Gietl, C., and Hock, B., 1986, Import of glyoxysomal malate dehydrogenase precursor into glyoxysomes: A heterologous in-vitro system. Planta 167:87-93.

Gillepsie, L. L., 1987, Identification of an outer mitochondrial membrane protein that interacts with a synthetic signal peptide. J. Biol. Chem. 262:7939-7942.

Gillepsie, L. L., Argan, C., Taneja, A. T., Hodges, R. S., Freeman, K. B., and Shore, G. C., 1985, A synthetic signal peptide blocks import of precursor proteins destined for the mitochondrial inner membrane matrix. J. Biol. Chem. 260:16045-16048.

Gilmore, R., and Blobel, G., 1983, Transient involvement of signal recognition particle and its receptor in the microsomal membrane prior to protein translocation. Cell 35:667-685.

Gilmore, R., and Blobel, G., 1985, Translocation of secretory proteins across the microsomal membrane occurs through an environment accessible to aqueous perturbants. Translocation through proteinaceous channel. Cell 42:497-505.

Gilmore, R., Walter, P., and Blobel, G., 1982, Protein translocation across the endoplasmic reticulum. II. Isolation and characterization of the signal recognition particle receptor. J. Cell Biol. 95(2, Pt. 1): 470-477.

Godinot, C., and Lardy, H. A., 1973, Biosynthesis of glutamate dehydrogenase in rat liver. Demonstration of its microsomal localization and hypothetical mechanism of transfer to mitochondria. Biochemstry 12:2051-2061. 
Gogol, E. P., and Engelman, D. M., 1984, Neutron scattering shows that cytochrome $b_{5}$ penetrates deeply the lipid bilayer. Biophys. J. 46:491-495.

Goldman, B. M., and Blobel, G., 1978, Biosynthesis of peroxisomes: Intracellular site of synthesis of catalase and uricase. Proc. Natl. Acad. Sci. U.S.A. 75:5066-5070.

Gonzalez, E., 1986, Glycoproteins in the matrix of glyoxysomes in endosperm of castor bean seedlings. Plant Physiol. 80:950-955.

Gonzalez, F. J., and Kasper, C. B., 1980, In vitro translation of epoxide hydratase messenger RNA. Biochem. Biophys. Res. Commun. 93:1254-1258.

Gonzalez-Cadavid, N. F., and Cardova, C. S., 1974, Role of membrane bound and free polysomes in the synthesis of cytochrome $c$ in rat liver. Biochem. J. 140:157-167.

Goodman, J. M., 1985, Dihydroxyacetone synthase is an abundant constituent of the methanolinduced peroxisomes of Candida boidinii. J. Biol. Chem. 260:7108-7113.

Goodman, J. M., Scott, C. W., Donahue, P. N., and Atherthon, J. P., 1984, Alcohol dehydrogenase assembles post-translationally into the peroxisomes of Candida boidinni. J. Biol. Chem. 259:8485-8493.

Gordon, J. I., Alpers, D. H., Schonfeld, G., Andy, R., Smith, D. P., and Strauss, A., 1981, The primary translation product of rat intestinal apo $\mathrm{A}_{1}$ mRNA is a preprotein. Fed. Proc. 40: 1635.

Gorgas, K., 1985, Serial section analysis of mouse hepatic peroxisomes. Anat. Embryol. 172:2132.

Gorin, M. B., Yancey, S. B., Cline, J., Revel, J. P., and Horwitz, J., 1984, The major intrinsic protein (MIP) of the bovine lens fiber membrane: Characterization and structure based on cDNA cloning. Cell 39:49-59.

Gould, S. J., Keller, G. A., and Subramani, S., 1987, Identification of a peroxisomal targeting signal at the carboxy terminus of firefly luciferase, J. Biol. Chem. 105:2923-2931.

Greenhut, S. F., and Roseman, M. A., 1985, Distribution of cytochrome $b_{5}$ between sonicated phospholipid vesicles of different sizes. J. Biol. Chem. 260:5883-5886.

Grossman, A., Bartlett, S. G., and Chua, N. H., 1980, Energy dependent uptake of cytoplasmically synthesized polypeptides by chloroplasts. Nature (London)285:625-628.

Grossman, A. R., Bartlett, S. G., Schmidt, G. W., Mullet, J. E., and Chua, N. H., 1982, Optimal conditions for post-translational uptake of proteins by isolated chloroplasts. In vitro synthesis and transport of plastocyanin, ferredoxin-NADP ${ }^{+}$oxidoreductase and fructose 1,6-diphosphatase, J. Biol. Chem. 257:1558-1563.

Guan, J. L., and Rose, J. K., 1984, Conversion of a secretory protein into a transmembrane protein results in its transport to the Golgi complex but not to the cell surface. Cell 37:779787.

Gundelfinger, E. D., Krause, E., Melli, M., and Dobberstein, B., 1983, The organization of 7 SL RNA in the signal recognition particle. Nucleic Acids Res. 11:7363-7374.

Halegoua, S., and Inouye, M., 1979, Translocation and assembly of outer membrane proteins of Escherichia coli. Selective accumulation of precursor and novel assembly intermediates caused by phenethyl alcohol. J. Mol. Biol. 130:30-61.

Halegoua, S., Hirashima, A., Sekizawa, J., and Inouye, M., 1976, Protein synthesis in toluenetreated Escherichia coli. Exclusive synthesis of membrane protein. Eur. J. Biochem. 69:163167.

Halegoua, S., Sekizawa, J., and Inouye, M., 1977, A new form of structural lipoprotein of outer membrane of Escherichia coli. J. Biol. Chem. 252:2324-2330.

Hallermeyer, G., and Neupert, W., 1976, Studies on the synthesis of mitochondrial proteins in the cytoplasm and on their transport in the mitochondria, in Genetics and Biogenesis of Chloroplasts and Mitochondria (T. Bücher, W. Neupert, W. Sebald, and S. Werner, eds.), pp. 807812, North-Holland/Elsevier, Amsterdam. 
Hallermeyer, G., Zimmerman, R., and Neupert, W., 1977, Kinetic studies on the transport of cytoplasmically synthesized proteins into mitochondria in intact cells of Neurospora crassa. Eur. J. Biochem. 81:523-532.

Hampsey, D. M., Lewin, A. S., and Kohlhaw, G. B., 1983, Submitochondrial localization, cell free synthesis and mitochondrial import of 2-isopropylmalate synthase of yeast. Proc. Natl. Acad. Sci. U.S.A. 80:1270-1274.

Hansen, W., Garcia, P. D., and Walter, P., 1986, In vitro protein translocation across the yeast endoplasmic reticulum. ATP dependent post-translational translocation of the prepro- $\alpha$-factor. Cell 45:397-406.

Harmey, M. A., Hallermeyer, G., and Neupert, W., 1976, In vitro synthesis and transport into mitochondria of cytoplasmically translated proteins, in Genetics and Biogenesis of Chloroplasts and Mitochondria (T. Bücher, W. Neupert, W. Sebald, and S. Werner, eds.), pp. 813818, North-Holland/Elsevier, Amsterdam.

Harmey, M. A., Hallermeyer, G., Korb, H., and Neupert, W., 1977, Transport of cytoplasmically synthesized proteins into mitochondria in a cell free system from Neurospora crassa. Eur. $J$. Biochem. 81:533-544.

Harnik-Ort, V., Prakash, K., Marcantonio, E., Colman, D. R., Rosenfeld, M. G., Adesnik, M., and Sabatini, D. D., 1987, Isolation and characterization of cDNA clones for rat ribophorin. I: Complete coding sequence and in vitro synthesis and insertion of the encoded product into endoplasmic reticulum. J. Cell Biol. 104:855-863.

Harold, F. M., and Van Brunt, J., 1977, Circulation of $\mathrm{H}^{+}$and $\mathrm{K}^{+}$across the plasma membrane is not obligatory for bacterial growth. Science 197:372-373.

Harson, M. M., Conder, M. J., and Lord, J. M., 1983, Endoplasmic reticulum and glyoxysomal membranes from castor bean endosperm: Interaction between membrane glycoproteins and organelle matrix proteins. Planta 157:143-149.

Hartl, F. U., and Just, W. W., 1987, Integral membrane polypeptides of rat liver peroxisomes: Topology and response to different metabolic states. Arch. Biochem. Biophys. 255:109119.

Hartl, F. U., Pfanner, N., and Neupert, W., 1987a, Translocation intermediates on the import pathway of proteins into mitochondria. Biochem. Soc. Trans. 15:96-97.

Hartl, F. U., Ostermann, J., Guiard, B., and Neupert, W., 1987b, Successive translocation into and out of the mitochondrial matrix: targeting of proteins in the intermembrane space by bipartite signal peptide, Cell 51:1027-1037.

Hase, T., Mueller, U., Riezman, H., and Schatz, G., 1984, A 70-kd protein of the yeast mitochondrial outer membrane is targeted and anchored via its extreme amino terminus. EMBO J. 3:3157-3164.

Hase, T., Nakai, M., and Matsubara, H., 1986, The N-terminal 21 amino acids of the $70 \mathrm{kd}$ protein of the yeast mitochondrial outer membrane direct $E$. coli $\beta$-galactosidase into mitochondrial matrix space in yeast cells. FEBS Lett. 197:199-203.

Hashimoto, T., 1982, Individual proxisomal $\beta$-oxidation enzymes. Ann. N.Y. Acad. Sci. 385:512.

Heinemann, F. S., and Ozols, J., 1984, The covalent structure of hepatic microsomal epoxide hydrolase, II. The complete amino acid sequence. J. Biol. Chem. 259:797-804.

Heinrich, P. C., Schmelzer, E., Northmann, C. K., and Witt, I., 1982, Rat liver cytochrome $c$ oxidase subunits IV and V; cell free synthesis as larger molecular weight precursors, mRNA sizes and sites of synthesis. Prog. Clin. Biol. Res. 102B:149-159.

Henning, B., and Neupert, W., 1981, Assembly of cytochrome $c$. Apocytochrome $c$ is bound to specific sites on mitochondria before its conversion to holocytochrome $c$. Eur. J. Biochem. 121:203-212.

Henning, B., Koehler, H., and Neupert, W., 1983, Receptor sites involved in post-translational 
transport of apocytochrome $c$ into mitochondria. Specificity, affinity and number of sites. Proc. Natl. Acad. Sci. U.S.A. 80:4963-4967.

Henning, U., Cole, S. T., Bremer, E., Hindennach, I., and Schaller, H., 1983, Gene fusion using OmpA gene coding for a major outer membrane protein of Escherichia coli. Eur. J. Biochem. 136:233-240.

Hernandez-Yago, J., and Grisolia, S., 1987, Apocytochrome c competes with pre-ornithine carbamyl transferase for transport into mitochondria, Biochem. Biophys. Res. Commun. 146:13181323.

Hernandez-Yago, J., Knecht, E., Felipo, V., Miralles, V., and Grisolia, S., 1983, Exit of proteins and fragments thereof from mitochondria is accelerated by the import of cytosolic synthesized protein. Biochem. Biophys. Res. Commun. 113:199-204.

Hiatt, A., McDonough, A., and Edelman, I. S., 1984, Assembly of the $\left(\mathrm{Na}^{+}+\mathrm{K}^{+}\right)$-adenosine triphosphatase, post-translational membrane integration of the $\alpha$-subunit. J. Biol. Chem. 259:2629-2635.

Higgins, C. F., Haag, P. D., Nikaido, K., Ardelshir, F., Garcia, G., and Ames, G. F. L., 1982, Complete nucleotide sequence and identification of membrane components of histidine transport operon of $S$. typhimurium. Nature (London) 298:723-727.

Highfield, P. E., and Ellis, R. J., 1978, Synthesis and transport of the small subunit of chloroplast ribulose bisphosphate carboxylase. Nature (London) 271:420-424.

Hock, B., and Gietl, C., 1982, Cell-free synthesis of watermelon glyoxysomal malate dehydrogenase: A comparison with the mitochondrial isoenzyme. Ann. N.Y. Acad. Sci. 386:350361 .

Holland, E. C., Leung, J. O., and Dirckamer, K., 1984, Rat liver asialoglycoprotein receptor lacks a cleavable $\mathrm{NH}_{2}$-terminal signal sequence. Proc. Natl. Acad. Sci. U.S.A. 81:73387342 .

Horiuchi, S., Marty-Mazars, D., Tai, P., and Davis, B. D., 1983a, Localization and quantitation of proteins characteristic of the complex membrane of Bacillus subtilis. J. Bacteriol. 153:12151221 .

Horiuchi, S., Tai, P., and Davis, B. D., 1983b, A 64-kilodalton membrane protein of Bacillus subtilis covered by secreting ribosomes. Proc Natl. Acad. Sci. U.S.A. 80:3287-3291.

Hortin, G., and Boime, I., 1980a, Inhibition of preprotein processing in ascites tumor lysates by incorporation of leucine analog. Proc. Natl. Acad. Sci. U.S.A. 77:1356-1360.

Hortin, G., and Boime, I., 1980b, Pre-prolactin accumulates in rat pituitary cells incubated with threonine analog. J. Biol. Chem. 255:7051-7053.

Hortsch, M., and Meyer, D. I., 1988, The human docking protein does not associate with the membrane of the rough endoplasmic reticulum via a signal or insertion sequence-mediated mechanism, Biochem. Biophys. Res. Commun. 150:111-117.

Hortsch, M., Avossa, A., and Meyer, D. I., 1985, A structural and functional analysis of docking protein: Characterization of active domains by proteolysis and specific antibodies. J. Biol. Chem. 260:9137-9145.

Hortsch, M., Avossa, D., and Meyer, D. I., 1986, Characterization of secretory protein translocation: Ribosome-membrane interaction in endoplasmic reticulum. J. Cell Biol. 103:241253.

Horwich, A. L., Fenton, W. A., Williams, K. R., Kalousek, F., Kraus, J. P. Doolittle, R. F., Konigsberg, W., and Rosenberg, L. E., 1984, Structure and expression of a complementary DNA for the nuclear coded precursor of human mitochondrial ornithine transcarbamylase. Science 224:1068-1074.

Horwich, A. L., Fenton, W. A., Firgaira, F. A., Fox, J. E., Kolansky, D., Mellman, I. S., and Rosenberg, L. E., 1985a, Expression of amplified DNA sequences for ornithine transcarbam- 
ylase in HeLa cells: Arginine residues may be required for mitochondrial import of enzyme precursor. J. Cell Biol. 100:1515-1521.

Horwich, A. L., Kalousek, F., and Rosenberg, L. E., 1985b, Arginine in the leader peptide is required for both import and proteolytic cleavage of a mitochondrial precursor. Proc. Natl. Acad. Sci. U.S.A. 82:4930-4933.

Horwich, A. L., Kalousek, F., Mellman, I., and Rosenberg, L. E., 1985c, A leader peptide is sufficient to direct mitochondrial import of a chimeric protein. EMBO J. 4:1129-1135.

Horwich, A. L., Kalousek, F., Fenton, W. A., Pollock, R. A., and Rosenberg, L. E., 1986, Targeting of pre-ornithine transcarbamylase to mitochondria: Definition of critical regions and residues in the leader peptide. Cell 44:451-459.

Horwich, A. L., Kalousek, F., Fenton, W. A., Furtak, K., Pollock, R. A., and Rosenberg, L. E., 1987, The ornithine transcarbamylase leader peptide directs mitochondrial import through both midportion structure and net positive charge. J. Cell Biol. 105:669-677.

Hu, V. W., and Wiesnieski, B. J., 1979, Photoactive labeling of $\mathrm{M}_{13}$ coat protein in model membranes by use of glycolipid probe. Proc. Natl. Acad. Sci. U.S.A. 76:5460-5464.

Hurt, E. C., and Schatz, G., 1987, A cytosolic protein contains a cryptic mitochondrial targeting signal. Nature (London) 325:499-503.

Hurt, E. C., and Van Loon, A. P. G. M., 1986, How proteins find mitochondria and intramitochondrial components. Trends Biochem. Sci. 11:204-207.

Hurt, E. C., Pesold-Hurt, B., and Schatz, G., 1984, The amino terminal region of an imported mitochondrial precursor polypeptide can direct cytoplasmic dehydrofolate reductase into the mitochondrial matrix. EMBO J. 3:3149-3156.

Hurt, E. C., Mueller, U., and Schatz, G., 1985, The first twelve amino acids of a yeast mitochondrial outer membrane protein can direct a nuclear encoded cytochrome oxidase subunit to the mitochondrial inner membrane. EMBO J. 4:3509-3518.

Hurt, E. C., Goldschmidt-Clermont, M., Pesold-Hurt, B., Rochaix, J. D., and Schatz, G., 1986a, A mitochondrial presequence can transport a chloroplast encoded protein into yeast mitochondria. J. Biol. Chem. 261:11440-11443.

Hurt, E. C., Soltanifar, N., Goldschmidt-Clermont, M., Rochaix, J. D., and Schatz, G., 1986b, The cleavable pre-sequence of an imported chloroplast protein directs attached polypeptides into yeast mitochondria. EMBO J. 5:1343-1350.

Ibrahimi, I., 1987, Dithiothreitol and the translocation of preprolactin across mammalian endoplasmic reticulum, J. Cell. Biol. 105:1555-1560.

Ibrahimi, I., and Gentz, R., 1987, A functional interaction between the signal peptide and the translation apparatus is detected by the use of a single point mutation which blocks translocation across mammalian endoplasmic reticulum. J. Biol. Chem. 262:10189-10194.

Ibrahimi, I., Culter, D., Stueber, D., and Bujard, H., 1986, Determinants for protein translocation across mammalian endoplasmic reticulum. Membrane insertion of truncated and full-length prelysozyme molecule, Eur. J. Biochem. 155:571-576.

Ichihara, S., Hussain, M., and Mizushima, S., 1982, Mechanism of export of outer membrane lipoproteins through the cytoplasmic membrane in Escherichia coli. Binding of lipoprotein precursors to the peptidoglycan layer. J. Biol. Chem. 257:495-500.

Ikeda, Y., Keese, S. M., Fenton, W. A., and Tanaka, K., 1987, Biosynthesis of four rat liver mitochondrial acyl-CoA dehydrogenases: In vitro synthesis, import into mitochondria and processing of their precursors in a cell free system and in cultured cells. Arch. Biochem. Biophys. 252:662-674.

Innis, M. A., Tokunaga, M., Williams, M. E., Loranger, J. M., Chang, S. Y., Chang, S., and Wu, H. C., 1984, Nucleotide sequence of Escherichia coli prolipoprotein signal peptidase (1 sp) gene. Proc. Natl. Acad. Sci. U.S.A. 81:3708-3712. 
Inouye, H., and Beckwith, J., 1977, Synthesis and processing of an Escherichia coli alkaline phosphatase precursor in vitro. Proc. Natl. Acad. Sci. U.S.A. 74:1440-1444.

Inouye, H., Barnes, W., and Beckwith, J., 1982, Signal sequence of alkaline phosphatase of $E$. coli. J. Bacteriol. 149:434-439.

Inouye, M., and Halegoua, S., 1980, Secretion and membrane localizaiton of proteins of Escherichia coli. CRC Crit. Rev. Biochem. 7:339-371.

Inouye, M., DiRienzo, J., Maeda, T., Movva, R., Nakamura, K., Lee, A. G., Pirtle, R., and Pirtle, I., 1980, Secretion of outer membrane proteins of Escherichia coli across the cytoplasmic membrane. Ann. N.Y. Acad. Sci. 343:362-367.

Inouye, S., Soberon, X., Franceschini, T., Nakamura, K., Itakura, K., and Inouye, M., 1982, Role of positive charge on the amino terminal region of the signal peptide in protein secretion across the membrane. Proc. Natl. Acad. Sci. U.S.A. 79:3438-3441.

Inouye, S., Hsu, C. P. S., Itakura, K., and Inouye, M., 1983a, Requirement for signal peptide cleavage of Escherichia coli lipoprotein. Science 221:59-61.

Inouye, S., Franceschini, T., Sato, M., Itakura, K., and Inouye, M., 1983b, Prolipoprotein signal peptidase of Escherichia coli requires a cysteine residue at the cleavage site. EMBO J. 2:8791.

Inouye, S., Duffaud, G., and Inouye, M., 1986, Structural requirement at the cleavage site for efficient processing of the lipoprotein secretory precursor of Escherichia coli. J. Biol. Chem. 261:10970-10975.

Inukai, M., and Inouye, M., 1983, Association of the prolipoprotein accumulated in the presence of globomycin with the outer membrane of Escherichia coli. Eur. J. Biochem. 130:27-32.

Ito, A., Ogishima, T., Ou, W., Omura, T., Aoyagi, H., Lee, S., Mihara, H., and Izumiya, N., 1985, Effects of synthetic model peptides resembling the extension peptides of mitochondrial enzyme precursors on import of the precursors into mitochondria. J. Biochem. (Tokyo) 98:15711582.

Ito K., 1984, Identification of the sec Y (prl A) gene product involved in protein export in Escherichia coli. Mol. Gen. Genet. 197:204-208.

Ito, K., Bassford, P. J. Jr., and Beckwith, J., 1981, Protein localization in E. coli. Is there a common step in insertion of periplasmic and outer membrane proteins? Cell 24:707-717.

Ito, K., Cerretti, D. P., Nashimoto, H., and Nomura, M., 1984, Characterization of an amber mutation in the structural gene for ribosomal protein $\mathrm{L}_{15}$; which impairs the expression of the protein export gene, sec Y, in Escherichia coli. EMBO J. 3:2319-2324.

Jain, M., and Zakim, D., 1987, The spontaneous incorporation of proteins into preformed bilayers, Biochim. Biophys. Acta 906:33-68.

Jaussi, R., Cotton, B., Juretic, N., Christen, P., and Schuemperli, D., 1985, The primary structure of the precursor of chicken mitochondrial aspartate aminotransferase. Cloning and sequence analysis of cDNA. J. Biol. Chem. 260:16060-16063.

Johnson, L. V., Walsh, M. L., Bockus, B. J., and Chen, L. B., 1981, Monitoring of relative mitochondrial membrane potential in living cells by fluorescence microscopy. J. Cell Biol. 88:526-535.

Josefsson, L. G., and Randall, L. L., 1981a, Processing in vivo of precursor maltose-binding protein in Escherichia coli occurs post-translationally as well as co-translationally. J. Biol. Chem. 256:2504-2507.

Josefsson, L. G., and Randall, L. L., 1981b, Different exported proteins in Excherichia coli show differences in the temporal mode of processing in vivo. Cell 25:151-157.

Joste, V., Berrez, J. C., Latruff, N., and Nelson, B. D., 1987, An import factor in reticulocyte lysates which stimulates processing of several precursors destined for the rat liver mitochondrial inner membrane, Acta Chem. Scand. Ser. B. B 41:770-772.

Kadonaga, J. T., Gantier, A. E., Strauss, D. R., Charles, A. D., Edge, M. D., and Knowles, J. 
R., 1984, The role of the $\beta$-lactamase signal sequence in the secretion of proteins by Escherichia coli. J. Biol. Chem. 259:2149-2154.

Kaiser, C. A., Preuss, D., Grisafi, P., and Botstein, D., 1987, Many random sequences funtionally replace the secretion signal sequences of yeast invertase. Science 235:312-317.

Kalousek, F., Orsulak, M. D., and Rosenberg, L. E., 1984, Newly processed transcarbamylase subunits are assembled to trimers in rat liver mitochondria. J. Biol. Chem. 259:5392-5395.

Kamisaki, Y., Sakakibara, R., Horio, Y., and Wada, H., 1982, Low isoelectric point of precursor of mitochondrial glutamic oxaloacetate transaminase isoenzyme synthesized in vitro. Biochem. Int. 4:289-296.

Kaput, J., and Blobel, G., 1986, Binding of the heme ligand inhibits translocation of in vitro synthesized cytochrome $c$ peroxidase into yeast mitochondria. Yeast 2:S182.

Kaput, J., Goltz, S., and Blobel, G., 1982, Nucleotide sequence of the yeast nuclear gene for cytochrome $c$ peroxidase precursor. Functional implications of the presequence for protein transport into mitochondria. J. Biol. Chem. 257:15054-15058.

Karlin-Neumann, G. A., and Tobin, E. M., 1986, Transit peptides of nuclear-encoded chloroplast proteins share a common amino acid framework. EMBO J. 5:9-13.

Kartenbeck, J., and Franke, W. W., 1974, Membrane relationship between endoplasmic reticulum and peroxisomes in rat hepatocytes and Morris hepatoma cells. Cytobiology 10:152-156.

Kasper, C. B., and Porter, T. D., 1985, Coding nucleotide sequence of rat NADPH-cytochrome P-450 oxidoreductase cDNA and identification of flavin binding domain, Proc. Natl. Acad. Sci. U.S.A. 82:973-977.

Katz, F. N., Rothman, J. E., Lingappa, V. R., Blobel, G., and Lodish, H. F., 1977, Membrane assembly in vitro: Synthesis, glycosylation and asymmetric insertion of transmembrane proteins. Proc. Natl. Acad. Sci. U.S.A. 74:3278-3282.

Kawajiri, K., Harano, T., and Omura, T., 1977, Biosynthesis of the mitochondrial matrix enzyme, glutamate dehydrogenase, in rat liver cells. II. Significance of binding of glutamate dehydrogenase to microsomal membrane. J. Biochem. (Tokyo) 82:1417-1423.

Kellems, R. E., Allison, V. F., and Butow, R. A., 1974, Cytoplasmic type 80 S ribosomes associated with yeast mitochondria. II. Evidence for the association of cytoplasmic ribosomes with the outer mitochondrial membrane. in situ. J. Biol. Chem. 249:3297-3303.

Kellems, R. E., Allison, V. F., and Butow, R. A., 1975, Cytoplasmic type 80 S ribosomes associated with yeast mitochondria. IV. Attachment of ribosomes to the outer membrane of isolated mitochondria. J. Cell Biol. 65:1-14.

Keller, G. A., Barton, M. C., Shapiro, D. J., and Singer, S. J., 1985, 3-Hydroxy-3-methyl glutaryl CoA reductase is present in peroxisomes in rat liver cells. Proc. Natl. Acad. Sci. U.S.A. 82:770-774.

Keller, G. A., Pazirandeh, M., and Krisans, S., 1986, 3-Hydroxyl-3-methyl glutaryl coenzyme A reductase localization in rat liver peroxisomes and microsomes of control and cholestyraminetreated animals: Quantitative biochemical and immunoelectron microscopical analyses. J. Cell Biol. 103:875-886.

Keller, G. A., Gould, S., Deluca, M., and Subramani, S., 1987, Firefly luciferase is targeted to peroxisomes in mammalian cells. Proc. Natl. Acad. Sci. U.S.A. 84:3264-3265.

Kendall, D. A., Bock, S. C., and Kaiser, E. T., 1986, Idealization of the hydrophobic segment of the alkaline phosphatase signal peptide. Nature (London) 321:706-708.

Keng, T., Alani, E., and Guarente, L., 1986, The nine aminoterminal residues of $\delta$-aminolevulinate synthase direct- $\beta$-galactosidase into the mitochondrial matrix. Mol. Cell. Biol. 6:355364.

Kensil, C. R., and Strittmatter, P., 1986, Binding and fluorescence properties of the membrane domain of NADH-cytochrome $b_{5}$ reductase. Determination of the depth of Trp-16 in the bilayer. J. Biol. Chem. 261:7316-7321. 
Kensil, C. R., Hediger, M. A., Ozols, J., and Strittmatter, P., 1983, Isolation and partial characterization of the amino-terminal membrane binding domain of NADH-cytochrome $b_{5}$ reductase. J. Biol. Chem. 258:14656-14663.

Kiino, D. R., and Silhavy, T. J., 1984, Mutation Prl $F_{1}$ relieves the lethality associated with export of $\beta$-galactosidase hybrid proteins in Escherichia coli. J. Bacteriol. 158:878-883.

Kindl, H., Köller, W., and Frevert, J., 1980, Cytoplasmic precursor pool during glyoxysome biosynthesis. Hoppe-Seyler's Z. Physiol. Chem. 361:465-467.

Kinoshita, N., Unemoto, T., and Kobayashi, H., 1984, Proton motive force is not obligatory for growth of Escherichia coli. J. Bacteriol. 160:1074-1077.

Kirwin, P. M., Elderfield, P. D., and Robinson, C., 1987, Transport of proteins into chloroplasts, Partial purification of thylakoidal processing peptidase involved in plastocyanin biogenesis, $J$. Biol. Chem. 262:16386-16390.

Kleene, R., Pfanner, N., Pfaller, R., Link, T. A., Sebald, W., Neupert, W., and Tropschug, M., 1987, Mitochondrial porin of Neurospora crassa: cDNA cloning, in vitro expression and import into mitochondria, EMBO J. 6:2627-2633.

Kloppstech, K., and Bitsch, A., 1986, Cross-linking of envelope proteins presumably involved in binding of nuclear coded chloroplast precursor proteins. Plant Biol. 2:235-240.

Koester, A., Heisig, M., Heinrich, P. C., and Just, W. W., 1986, In vitro synthesis of peroxisomal membrane polypeptides. Biochem. Biophys. Res. Commun. 137:628-632.

Kohorn, B. D., Harel, E., Chitnis, P. R., Thornber, J. P., and Tobin, E. M., 1986, Functional and mutational analysis of the light-harvesting chlorophyll $\mathrm{a} / \mathrm{b}$ protein of thylakoid membranes. J. Cell Biol. 102:972-981.

Kolansky, D. M., Conboy, J. G., Fenton, W. A., and Rosenberg, P. E., 1982, Energy dependent translocation of precursor of ornithine transcarbamylase by isolated rat liver mitochodria. $J$. Biol. Chem. 257:8467-8471.

Köller, W., and Kindl, H., 1980, Cytosolic malate synthethase. A small pool characterized by rapid turnover. Hoppe-Seyler's Z. Physiol. Chem. 361:1437-1444.

Kopito, R. R., and Lodish, H. F., 1985, Primary structure and transmembrane orientation of the murine anion exchange protein. Nature (London) 316:234-238.

Kornfeld, R., and Kornfeld, S., 1985, Assembly of asparagine-linked oligosaccharides. Annu. Rev. Biochem. 54:631-664.

Koshland, D., and Botstein, D., 1980, Secretion of $\beta$-lactamase requires the carboxy end of the protein. Cell 20:749-760.

Koshland, D., and Botstein, D., 1982, Evidence for post-translational translocation of $\beta$-lactamase across the bacterial inner membrane. Cell 30:893-902.

Koshland, D., Sauer, R. T., and Botstein, D., 1982, Diverse effects of mutations in the signal sequence on the secretion of $\beta$-lactamase in Salmonella typhimurium. Cell. 30:903-914.

Kreibich, G., Ullrich, B. L., and Sabatini, D. D., 1978a, Proteins of rough microsomal membranes related to ribosome binding. I. Identification of ribophorins I and II, membrane proteins characteristic of rough microsomes. J. Cell Biol. 77:464-487.

Kreibich, G., Freienstein, C. M., Pereyra, P. N., Ullrich, B. L., and Sabatini, D. D., 1978b, Proteins of rough microsomal membranes related to ribosome binding. II. Cross-linking of bound ribosomes to specific membrane proteins exposed at the binding site. J. Cell Biol. 77:488-506.

Kreibich, G., Csako-Graham, M., Grebeneau, R., and Sabatini, D. D., 1980, Functional and structural characteristics of endoplasmic reticulum membrane proteins associated with ribosome binding sites. Ann N.Y. Acad. Sci. 343:17-33.

Kreibich, G., Ojakian, G., Rodriguez-Boulan, E. J., and Sabatini, D. D., 1982, Recovery of ribophorins in "inverted rough" vesicles derived from liver rough microsomes. J. Cell Biol. 93:111-121. 
Krieg, U. C., Walter, P., and Johnson, A. E., 1986, Photo cross-linking of the signal sequence of nascent preprolactin to the 54-kilodalton polypeptide of the signal recognition particle. Proc. Natl. Acad. Sci. U.S.A. 83:8604-8608.

Kruse, C., and Kindl, H., 1983, Oligomerization of malate synthase during glyoxysome biosynthesis. Arch. Biochem. Biophys. 223:629-638.

Kruse, C., Frevert, J., and Kindl, H., 1981, Selective uptake by glyoxysome of in vitro translated malate synthase. FEBS. Lett. 129:36-38.

Kuhn, A., Wickner, W., and Kreil, G., 1986, The cytoplasmic terminus of $\mathbf{M}_{13}$ procoat is required for the membrane insertion of its central domain. Nature (London) 322:335-339.

Kumamoto, C. A., and Beckwith, J., 1983, Mutations in a new gene, sec B, cause defective protein localization in Escherichia coli. J. Bacteriol. 154:253-260.

Kumamoto, C. A., Oliver, D. B., and Beckwith, J., 1984, Signal sequence mutations disrupt feedback between secretion of an exported protein and its synthesis in E. coli. Nature (London) 308:863-864.

Kumamoto, T., Morohashi, K., Ito, A., and Omura, T., 1987, Site-directed mutagenesis of basic amino acid residues in the extension peptide of P-450 (scc) precursor: effects on import of precursor into mitochondria, J. Biochem. (Tokyo) 102:833-834.

Kuntz, M., Simons, A., Schell, J., and Schreier, P. H., 1987, Targeting of protein to chloroplasts in transgenic tobacco by fusion to mutated transit peptide. Mol. Gen. Genet 205:454460.

Kurzchalia, T. V., Wiedmann, M., Girshovich, A. S., Bochkareva, E. S., Bielka, H., and Rapoport, T. A., 1986, The signal sequence of nascent preprolactin interacts with the $54 \mathrm{~K}$ polypeptide of signal recognition particle. Nature (London) 320:634-636.

Lai, J. S., Sarvas, M., Brammar, W. J., Neugebauer, K., and Wu, H. C., 1981, Bacillus licheniformis penicillinase synthesized in Escherichia coli contains covalently linked fatty acid and glyceride. Proc. Natl. Acad. Sci. U.S.A. 78:3506-3510.

Lamppa, G. K., Morelli, G., and Chua, N. H., 1985, Structure and developmental regulation of a wheat gene encoding the major chlorophyll a/b-binding polypeptide. Mol. Cell. Biol. 5:13701378.

Lauffer, L., Garcia, P. D., Harkins, R. N., Coussens, L., Axel, U., and Walter, P., 1985, Topology of signal recognition particle receptor in endoplasmic reticulum membrane. Nature (London) 318:334-338.

Lazarow, P. B., and De Duve, C., 1973, The synthesis and turnover of rat liver peroxisomes. V. Intracellular pathway of catalase synthesis. J. Cell Biol. 59:507-524.

Lazarow, P. B., Robbin, M., Fujiki, Y., and Wong, L., 1982, Biogenesis of peroxisomal proteins in vivo and in vitro. Ann. N.Y. Acad. Sci. 386:285-300.

Lee, C. A., and Beckwith, J., 1986, Suppression of growth and protein secretion defects in Escherichia coli sec A mutants by decreasing protein synthesis. J. Bacteriol. 166:878-883.

Lee, C. A., Fournier, M. J., and Beckwith, J., 1985, Escherichia coli 6S RNA is not essential for growth of protein secretion. J. Bacteriol. 161:1156-1161.

Leskes, A., Siekevitz, P., and Palade, G. E., 1971a, Differentiation of endoplasmic reticulum in hepatocytes. I. Glucose-6-phosphatase distribution in situ. J. Cell Biol. 49:264-287.

Leskes, A., Siekevitz, P., and Palade, G. E., 1971b, Differentiation of endoplasmic reticulum in hepatocytes. II. Glucose-6-phosphatase in rough microsomes. J. Cell Biol. 49:288-302.

Lewin, A. S., and Norman, D. K., 1983, Assembly of $F_{1}$-ATPase in isolated mitochondria. $J$. Biol. Chem. 258:6750-6755.

Liao, M. J., and Khorana, H. G., 1984, Removal of the carboxyl terminal peptide does not affect refolding or function of bacteriorhodopsin as a light-dependent proton pump. J. Biol. Chem. 259:4194-4199.

Lin, J. J. C., Kanazawa, H., Ozols, J., and Wu, H. C., 1978, An Escherichia coli mutant with 
an amino acid alteration within the signal sequence of outer membrane prolipoprotein. Proc. Natl. Acad. Sci. U.S.A. 75:4891-4895.

Lin, J. J. C., Kanazawa, H., and Wu, H. C., 1980, Assembly of outer membrane proteins in Escherichia coli mutant, with a single amino acid replacement within the signal sequence of prolipoprotein. J. Bacteriol. 141:550-557.

Lingappa, V. R., Katz, F. N., Lodish, H. F., and Blobel, G., 1978, A signal sequence for the insertion of a membrane glycoprotein. Similarity to the signals of secretory proteins in a primary structure and function. J. Biol. Chem. 253:8667-8670.

Lingappa, V. R., Lingappa, J. R., and Blobel, G., 1979, Chicken ovalbumin contains an internal signal sequence. Nature (London) 281:117-121.

Lingelbach, K. R., Graf, L. J., Dunn, A. R., and Hoogenraad, N. J., 1986, Effect of deletion within the leader peptide of pre-ornithine transcarbamylase on mitochondrial import. Eur. J. Biochem. 161:19-23.

Lipp, J., and Dobberstein, B., 1986a, Signal recognition particle-dependent membrane insertion of mouse invariant chain: A membrane-spanning protein with cytoplasmically exposed amino terminus. J. Cell Biol. 102:2169-2175.

Lipp, J., and Dobberstein, B., 1986b, The membrane-spanning segment of invariant chain (I $\gamma)$ contains a potentially cleavable signal sequence. Cell 46:1103-1112.

Lord, J. M, 1978, Evidence that proliferation of endoplasmic reticulum precedes formation of glyoxysomes and mitochondria in germinating castor bean endosperm. J. Exp. Bot. 29:1323.

Lord, J. M., and Roberts, L. M., 1982, Glyoxysome biosynthesis via endoplasmic reticulum in castor bean endosperm. Ann. N.Y. Acad. Sci. 386:362-376.

Lory, S., Tai, P. C., and Davis, B. D., 1983, Mechanism of protein secretion by gram-negative bacteria: Pseudomonas aeruginosa exotoxin A. J. Bacteriol. 156:695-702.

Lubben, T. H., Bansberg, J., and Keegstra, K., 1987, Stop-transfer regions do not halt translocation of proteins into chloroplasts, Science 238:1112-1114.

Lubben, T. H., and Keegstra, K., 1986, Efficient in vitro import of a cytosolic heat shock protein into pea chloroplasts. Proc. Natl. Acad. Sci. U.S.A. 83:5502-5506.

Lubin, I. M., Wu, L. N. Y., Wuthier, R. E., and Fisher, R. R., 1987, Rhodamine 123 inhibits import of rat liver mitochondrial transhydrogenase. Biochem. Biophys. Res. Commun. 144:477483.

Lunn, C. A., and Inouye, M., 1987, Effects of prolipoprotein signal peptide mutations on secretion of hybrid prolipo- $\beta$-lactamase in Escherichia coli. J. Biol. Chem. 262:8318-8324.

Lycett, G. W., Delauney, A. J., Catehouse, J. A., Gilroy, J., Croy, R. R. D., and Boutler, D., 1983, The vicilin gene family of pea (Pisum sativum L.) a complete cDNA sequence for preprovicilin. Nucleic Acids Res. 11:2367-2380.

Maarse, A. C., Van Loon, A. P. G. M., Riezman, H., Gregor, I., Schatz, G., and Grivell, L. A., 1984, Subunit IV of yeast cytochrome oxidase: Cloning and nucleotide sequencing of the gene and partial amino acid sequencing of the mature protein. EMBO J. 3:2831-2837.

Maccecchini, M. L., Rudin, Y., Blobel, G., and Schatz, G., 1979a, Import of proteins into mitochondria: Precursor forms of the extramitochondrially made $F_{1}$-ATPase subunits in yeast. Proc. Natl. Acad. Sci. U.S.A. 76:343-347.

Maccecchini, M. L., Rudin, Y., and Schatz, G., 1979b, Transport of proteins across the mitochondrial outer membrane. A precursor form of the cytoplasmically made intermembrane enzyme, cytochrome $c$ peroxidase. J. Biol. Chem. 254:7468-7471.

Maeshima, M., Yokoi, H., and Asahi, T., 1988, Evidence for no proteolytic processing during transport of isocitrate lyase into glyoxysomes in castor bean endosperm, Plant Cell Physiol. 29:381-384. 
Maher, P. S., and Singer, S. J., 1986, Disulfide bonds and the translocation of proteins across membranes. Proc. Natl. Acad. Sci. U.S.A. 83:9001-9005.

Majzoub, J. A., Rosenblatt, M., Fennick, B., Mannus, R., Kronenberg, H. M., Potts, J. T. Jr., and Habener, J. F., 1980, Synthetic pre-pro-parathyroid hormone leader sequence inhibits cell-free processing of placental, parathyroid and pituitary prehormones. J. Biol. Chem. 255:11478-11484.

Marcantonio, E., Amar-Costesec, A., and Kreibich, G., 1984, Segregation of polypeptide translocation apparatus to regions of the endoplasmic reticulum containing ribophorin and ribosomes. II. Rat liver microsomal subfractions contain equimolar amounts of ribophorins and ribosomes. J. Cell Biol. 99:2254-2259.

Martial, J. A., Hallwell, R. A., Baxter, J. D., and Goodman, H. M, 1979, Human growth hormone: Complementary DNA cloning and expression in bacteria. Science 205:602-607.

Marty-Mazars, D. M., Horiuchi, S., Tai, P. C., and Davis, B. D., 1983, Proteins of ribosomebearing and free-membrane domains in Bacillus subtilis. J. Bacteriol. 154:1381-1388.

Matocha, M. F., and Waterman, M. R., 1984, Discriminatory processing of the precursor forms of cytochrome P-450 scc and adrenodoxin by adrenocortical and heart mitochondria. J. Biol. Chem. 259:8672-8678.

Matocha, M. F., and Waterman, M. R., 1986, Import and processing of P-450 scc and P-450 $11 \beta$ precursors by corpus luteal mitochondria: A processing pathway recognizing homologous and heterologous precursors. Arch. Biochem. Biophys. 250:456-460.

Matsuura, S., Arpin, M., Hannun, C., Margoliash, E., Sabatini, D. D., and Morimoto, T., 1981, In vitro synthesis and post-translational uptake of cytochrome $c$ into isolated mitochondria: Role of a specific addressing signal in the apocytochrome c. Proc. Natl. Acad. Sci. U.S.A. 78:4368-4372.

McIntyre, S., Freudl, R., Degen, M., Hindennach, I., and Henning, U., 1987, The signal sequence of an $E$. coli outer membrane protein can mediate translocation of a not normally secreted protein across the plasma membrane. J. Biol. Chem. 262:8416-8422.

McKean, D. J., and Maurer, R. A., 1978, Complete amino acid sequence of the precursor region of rat prolactin. Biochemistry 17:5215-5219.

MacLennan, D. H., Brandl, C. J., Korczak, B., and Green, N. M., 1985, Amino-acid sequence of $\mathrm{Ca}^{2+}+\mathrm{Mg}^{2+}$-dependent ATPase from rabbit muscle sarcoplasmic reticulum, deduced from its complementary DNA sequence. Nature 316:696-700.

Meek, R. L., Walsh, K. A., and Palmiter, R. D., 1982, The signal sequence of ovalbumin is located near the aminoterminus. J Biol. Chem. 257:12245-12251.

Meyer, D. I., 1982, Translocation of secretory and membrane proteins across the endoplasmic reticulum: The requirement for two specific receptors. J. Cell Biol. 95(2, Pt. 2) 388a (Abstract).

Meyer, D. I., 1985, Signal recognition particle does not mediate a translational arrest of nascent secretory proteins in mammalian cell free systems. EMBO J. 4:2031-2033.

Meyer, D. I., and Dobberstein, B., 1980a, A membrane component essential for vectorial translocation of nascent proteins across the endoplasmic reticulum: Requirement for its extraction and reassociation with the membrane. J. Cell Biol. 87:498-502.

Meyer, D. I., and Dobberstein, B., 1980b, Identification and characterization of a membrane component essential for the translocation of nascent proteins across the membrane of the endoplasmic reticulum. J. Cell Biol. 87:503-508.

Meyer, D. I., Krause, E., and Dobberstein, B., 1982a, Secretory protein translocation across membranes. The role of the "docking protein." Nature (London) 297:647-650.

Meyer, D. I., Louvard, D., and Dobberstein, B., 1982b, Characterization of molecules involved in protein translocation using a specific antibody. J. Cell Biol: 92:579-582. 
Michaelis, S., Guarente, L., and Beckwith, J., 1983a, In vitro construction and characterization of Pho A-lac Z gene fusions in Escherichia coli. J. Bacteriol. 154:356-365.

Michaelis, S., Inouye, H., Oliver, D., and Beckwith, J., 1983b, Mutations that alter the signal sequences of alkaline phosphatase in Escherichia coli. J. Bacteriol. 154:366-374.

Mihara, K., and Blobel, G., 1982, In vitro synthesis and integration into mitochondria of porin, a major protein of the outer mitochondrial membrane of Saccharomyces cerevisiae, Proc. Natl. Acad. Sci. U.S.A. 79:7012-7016.

Mihara, K., and Sato, R., 1985, Molecular cloning and sequencing of cDNA from yeast porin, an outer mitochodrial membrane protein: A search for targeting signal in primary structure. $E M B O$ J. 4:769-774.

Mihara, K., Sato, R., Sakakibara, R., and Wada, H., 1978, Reduced nicotinamide adenine dinucleotide-cytochrome $b_{5}$ reductase: Location of the hydrophobic membrane binding region at the carboxyl terminal and the masked amino terminus. Biochemistry 17:2829-2834.

Mihara, K., Omura, T., Harano, T., Brenner, S. C., Fleischer, S., Rayagoplan, K. V., and Blobel, G., 1982, Rat liver L-glutamate dehydrogenase, malate dehydrongenase, D- $\beta$-hydroxybutyrate dehydrogenase and sulfite oxidase are each synthesized as larger precursors by cytoplasmic free polysomes. J. Biol. Chem. 257:3355-3358.

Milstein, C., Brownlee, G. G., Harrison, T. M., and Mathews, M. B., 1972, A possible precursor of immunoglobulin light chains. Nature New Biol. 239:117-120.

Minami, E., Shinohara, K., Kuwabara, T., and Watanabe, A., 1986, In vitro synthesis and assembly of photosystem II proteins of spinach chloroplasts. Arch. Biochem. Biophys. 244:517527.

Minsky, A., Summers, R. G., and Knowles, J. R., 1986, Secretion of $\beta$-lactamase into the periplasm of Escherichia coli: Evidence for a distinct release step associated with a conformation change. Proc. Natl. Acad. Sci. U.S.A. 83:4180-4184.

Mirales, V., Felipo, V., Hernandez-Yago, J., and Grisolia, S., 1983, Transport of the precursor for rat liver glutamate dehydrogenase into mitochondria in vitro. Biochem. Biophys. Res. Commun. 110:449-503.

Mishkind, M. L., Wessler, S. R., and Schmidt, G. W., 1985, Functional determinants in transit sequence: Import and partial maturation by vascular plant chloroplasts of the ribulose 1,5bisphosphate carboxylase small subunit of Chlamydomonas. J. Cell Biol. 100:226-234.

Miura, S., Mori, M., Morita, T., and Tatibana, M., 1982, A high isolectric point of mitochondrial ornithine carbamyltransferase precursor and inhibition of its processing in vitro by basic proteins. Biochem. Int. 4:201-208.

Miura, S., Mori, M., and Tatibana, M., 1983, Transport of ornithine carbamyltransferase precursor into mitochondria. Stimulation by potassium ion, magnesium ion and reticulocyte cytosolic protein(s). J. Biol. Chem. 258:6671-6674.

Miura, S., Mori, M., Takiguchi, M., Tatibana, M., Furuta, S., Miyazawa, S., and Hashimoto, T., 1984, Biosynthesis and intracellular transport of enzymes of peroxisomal $\beta$-oxidation. $J$. Biol. Chem. 259:6397-6402.

Miura, S., Amaya, Y., and Mori, M., 1986, A metalloprotease involved in the processing of mitochondrial precursor proteins. Biochem. Biophys. Res. Commun. 134:1151-1159.

Miyazawa, S., Ozasa, H., Furuta, S., Osumi, T., Hashimoto, T., Miura, S., Mori, M., and Tatibana, M., 1983, Biosynthesis and turnover of carnitine acetyltransferase in rat liver. $J$. Biochem. (Tokyo) 93:453-459.

Montisano, D. F., Cascarano, J., Pickett, C. B., and James, T. W., 1982, Association between mitochondria and rough endoplasmic reticulum in rat liver, Anat. Rec. 203:441-450.

Moore, K. E., and Miura, S., 1987, A small hydrophobic domain anchors leader peptidase to the cytoplasmic membrane of Escherichia coli. J. Biol. Chem. 262:8806-8813.

Mori, M., Morita, T., Ikeda, F., Amaya, Y., Tatibana, M., and Cohen, P. P., 1981, Synthesis, 
intracellular transport and processing of the precursor for mitochondrial ornithine transcarbamylase and carbamyl phosphate synthetase I in isolated hepatocytes. Proc. Natl. Acad. Sci. U.S.A. 78:6056-6060.

Mori, M., Miura, S., Morita, T., and Tatibana, M., 1982, Synthesis and intracellular transport of mitochondrial carbamyl phosphate synthetase I and ornithine transcarbamylase. Adv. Exp. Med. Biol. 153:267-276.

Mori, M., Matsue, H., Miura, S., Tatibana, M., and Hashimoto, T., 1985, Transport of matrix proteins into mitochondrial matrix. Evidence suggesting a common pathway for 3-ketoacylCoA thiolase and enzymes having presequences. Eur. J. Biochem 149:181-186.

Morita, T., Mori, M., Ikeda, F., and Tatibana, M., 1982, Transport of carbamyl phosphate synthetase I and ornithine transcarbamylase into mitochondria. Inhibition by rhodamine 123 and accumulation of enzyme precursors in isolated hepatocytes. J. Biol. Chem. 257:13075-13080.

Mueckler, M., and Lodish, H. F., 1986, Post-translational insertion of a fragment of the glucose transporter into microsomes requires phosphoanhydride bond cleavage. Nature (London) 322:549-552.

Mueckler, M., Caruso, C., Baldwin, S. A., Panico, M., Blench, I., Morris, H. R., Allard, W. J., Lienhard, G. E., and Lodish, H. F., 1985, Sequence and structure of a human glucose transporter. Science 229:941-945.

Mueller, M., and Blobel, G., 1984, Protein export in Escherichia coli requires a soluble activity. Proc. Natl. Acad. Sci. U.S.A. 81:7737-7741.

Mueller, M., Ibrahimi, I., Chang, C. N., Walter, P., and Blobel, G., 1982, A bacterial secretory protein requires signal recognition particle for translocation across mammalian endoplasmic reticulum. J. Biol. Chem. 257:11860-11863.

Müller, G., and Zimmermann, R., 1987, Import of honeybee prepromelittin into the endoplasmic reticulum: structural basis of independence of SRP and docking protein, EMBO J. 6:20992107.

Murén, E. M., and Randall, L. L., 1985, Export of $\alpha$-amylase by Bacillus amyloliquefaciens requires proton motive force. J. Bacteriol. 164:712-716.

Mustonen, P., Virtanen, J. A., Somerharju, P. J., and Kinnunen, P. K. J., 1987, Binding of cytochrome $c$ to liposomes as revealed by the quenching of fluorescence from pyrene-labeled phospholipids. Biochemistry 26:2991-2997.

Myers, M., Mayorga, D. L., Emtage, J., and Friere, E., 1987, Thermodynamic characterization of interactions between ornithine transcarbamylase leader peptide and phospholipid bilayer membranes, Biochemistry 26:4309-4315.

Nabi, N., Ishikawa, T., Ohashi, M., and Omura, T., 1983, Contribution of cytoplasmic free and membrane-bound ribosomes to the synthesis of NADPH-adrenodoxin of bovine adrenal cortex mitochondria. J. Biochem. (Tokyo) 94:1505-1515.

Nagaraj, R., 1984, Interaction of synthetic signal sequence fragments with model membranes. FEBS Lett 165:79-82.

Nagata, S., Tsunetsuga-Yokota, Y., Naito, A., and Kaziro, Y., 1983, Molecular cloning and sequence determination of the nuclear gene coding for mitochondrial elongation factor Tn of Saccharomyces cerevisiae. Proc. Natl. Acad. Sci. U.S.A. 80:6182-6196.

Nakagawa, T., Maeshima, M., Muto, H., Kajiura, H., Hattori, H., and Asahi, T., 1987, Separation, amino-terminal sequence and cell free synthesis of the smallest subunit of sweet potato cytochrome $c$ oxidase. Eur. J. Biochem. 165:303-307.

Natsoulis, G., Hilger, F., and Fink, G. R., 1986, The HTS gene encodes both the cytoplasmc and mitochondrial histidine tRNA synthetases of $S$. cerevisiae. Cell 46:235-243.

Nelson, N., Maccecchini, M. L., Rudin, Y., and Schatz, G., 1979, Import of proteins into mitochondria, in Biological Function of Proteinases (H. Holzer and H. Tschesche, eds.), pp. 109119 , Springer, Berlin. 
Nesmeyanova, M. A., 1982, On the possible participation of acid phospholipids in the translocation of secreted proteins through the bacterial cytoplasmic membrane, FEBS Lett. 142:189193.

Ness, S. A., and Weiss, R. L., 1987, Carboxyl-terminal sequences influence the import of mitochondrial protein precursors in vivo, Proc. Natl. Acad. Sci. USA 84:6692-6696.

Neupert, W., and Schatz, G., 1981, How proteins are transported into mitochondria. Trends Biochem. Sci. 6:1-4.

Nguyen, M., and Shore, G. C., 1987, Import of hybrid vesicular stomatitis G protein to the mitochondrial inner membrane. J. Biol. Chem. 262:3929-3931.

Nguyen, M., Argan, C., Lusty, C. J., and Shore, G. C., 1986, Import and processing of hybrid proteins by mammalian mitochondria in vitro. J. Biol. Chem. 261:800-805.

Nguyen, M., Argan, C., Sheffield, W. P., Bell, A. W., Shields, D., and Shore, G. C., 1987, A signal sequence domain essential for processing, but not import, of mitochondrial pre-ornithine carbamyltransferase. J. Cell Biol. 104:1193-1198.

Nicholson, D. W., Koehler, H., and Neupert, W., 1987, Import of cytochrome $c$ into mitochondria. Cytochrome $c$ heme lyase. Eur. J. Biochem. 144:147-157.

Niranjan, B. G., Raza, H., Shayiq, R. M., Jefcoate, C. R., and Avadhani, N. G., 1988, Hepatic mitochondrial cytochrome P-450 system. Identificatin and characterization of a precursor form of mitochondrial cytochrome P-450 induced by 3-methylcholanthrene, J. Biol.Chem. 262:575580.

Noda, M., Shimizu, S., Tanabe, T., Takai, T., Kayano, T., Ireka, T., Takahashi, H., Nakayama, H., Hanaoka, Y., Inayama, S., Hayashiba, H., Miyata, T., and Numa, S., 1984, Primary structure of Electrophorus electricus sodium channel deduced from cDNA sequence. Nature (London) 312:121-127.

Noel, C., Nicolau, Y., Argan, C., Rachubinski, R. A., and Shore, G. C., 1985, In vitro synthesis and assembly of a $68 \mathrm{k}$ Da outer membrane protein from rat liver. Biochim. Biophys. Acta 814:35-42.

Oda, T., Ichiyama, A., Miura, S., and Mori, M., 1984, Uptake and processing of serine: Pyruvate aminotransferase precursor by rat liver mitochondria in vitro and in vivo. J. Biochem. (Tokyo) 95:815-824.

Ogishima, T., Okada, Y., and Omura, T., 1985, Import and processing of the precursor of cytochrome P-450 (scc) by bovine adrenal cortex mitochondria. J. Biochem. 98:781-791.

Ohashi, A., Gibson, J., Gregor, I., and Schatz, G., 1982, Import of proteins into mitochondria. The precursor of cytochrome $c_{1}$ is processed in two steps one of them heme dependent. $J$. Biol. Chem. 257:13042-13047.

Ohba, M., and Schatz, G., 1987, Disruption of the outer membrane restores protein import to trypsin treated yeast mitochondria. EMBO J. 6:2117-2122.

Ohkawa, I., and Webster, R. E., 1981, The orientation of the major coat protein of bacteriophage $\mathrm{f}_{1}$ in the cytoplasmic membrane of Escherichia coli. J. Biol. Chem. 256:9951-9958.

Ohno-Iwashita, Y., and Wickner, W., 1983, Reconstitution of rapid and asymmetrric assembly of $\mathrm{M}_{13}$ procoat protein into liposomes which have bacterial leader peptidase. J. Biol. Chem. 258:1895-1900.

Okada, Y., Frey, A. B., Guenthner, T. M., Oesh, F., and Sabatini, D. D., 1982, Sites of synthesis and mode of insertion of cytochrome $b_{5}$, cytochrome $b_{5}$ reductase, cytochrome P-450 reductase and epoxide hydrolase. Eur. J. Biochem. 122:393-402.

Oliver, D. B., 1985, Identification of five new essential genes involved in the synthesis of a secreted protein in Escherichia coli. J. Bacteriol. 161:285-291.

Oliver, D. B., and Beckwith, J., 1981, E. coli mutant pleiotropically defective in the export of secreted proteins. Cell 25:765-772.

Oliver, D. B., and Beckwith, J., 1982a, Identification of a new gene (sec A) and gene product 
involved in the secretion of the envelope proteins in Escherichia coli. J. Bacteriol. 150:686691.

Oliver, D. B., and Beckwith, J., 1982b, Regulation of a membrane component is required for protein secretion in Escherichia coli. Cell 30:311-319.

Oliver, D. B., and Liss; L. R., 1985, PrlA-mediated suppression of signal sequence mutations is modulated by the sec A gene product of Escherichia coli K-12. J. Bacteriol. 161:817-819.

Ono, H., and Ito, A., 1984a, Transport of the precursor of sulfite oxidase into intermembrane space of liver mitochondria: Characterization of import and processing activities. J. Biochem. (Tokyo) 95:345-352.

Ono, H., and Ito, A., 1984b, Transport of the precursor for sulfite oxidase into intermembrane space of liver mitochondria: Binding of the precursor to outer mitochondrial membrane. $J$. Biochem. (Tokyo) 95:353-358.

Ono, H., and Tuboi, S., 1985, Partial purification of the receptor protein for import of preornithine aminotransferase into mitochondria. Biochem. Int. 10:351-357.

Ono, H., and Tuboi, S., 1986, Translocation of proteins into rat liver mitochondria. The precursor polypeptides of a large subunit of succinate dehydrogenase and ornithine aminotransferase and their import into their own locations of mitochondria. Eur. J. Biochem. 155:543-549.

Ono, H., and Tuboi, S., 1988, The cytosolic factor required for import of precursors of mitochondrial proteins into mitochondria, J. Biol. Chem. 263:3188-3193.

Ono, H., Yoshimura, N., Sato, M., and Tuboi, S., 1985, Translocation of proteins into rat liver mitochondria. Existence of two different precursor polypeptides of liver fumarase and import of the precursor into mitochondrria. J. Biol. Chem. 260:3402-3407.

Op den Kamp, J. A. F., 1979, Lipid asymmetry in membranes, Ann. Rev. Biochem. 48:47-71.

Osumi, T., Ishii, N., Hijikata, M., Kamijo, K., Ozasa, H., Furuta, S., Miyazawa, S., Kondo, K., and Inoue, K., 1985, Molecular cloning and nucleotide sequence of cDNA for rat peroxisomal enoyl CoA: Hydrarase-3-hydroxyacyl-CoA dehydrogenase bifunctional enzyme. $J$. Biol. Chem. 260:8905-8910.

Otha, S., and Schatz, G., 1984, A purified precursor polypeptide requires a cytosolic protein fraction for import into mitochondria. EMBO J. 3:651-657.

Ou, W., Ito, A., Morohashi, K., Fujii-Kuriyama, Y., and Omura, T., 1986, Processing-independent in vitro translocation of cytochrome P-450 (scc) precursor across mitochondrial membranes. J. Biochem. (Tokyo) 100:1287-1296.

Ovchinnikov, Yu. A., 1982, Rhodopsin and bacteriorhodopsin: Structure function relationship, FEBS Lett. 148:179-191.

Ovchinnikov, Yu. A., Abdulaev, N. G., Feigina, M. Y., Artamonov, I. D., Bogachuk, A. S., Zolotarev, A. S., Egamian, E. R., and Kostetsky, P. V., 1983, Visual rhodopsin III. Total amino acid sequence and arrangement in the membrane, Bioorg. Khim 9:1331-1340.

Ozasa, H., Furuta, S., Miyazawa, S., Osumi, T., Hashimoto, T., Mori, M., Miura, S., and Tatibana, M., 1984, Biosynthesis of enzymes of rat liver mitochondrial $\beta$-oxidation. Eur. J. Biochem. 144:453-458.

Pain, D., and Blobel, G., 1987, Protein import into chloroplasts requires a chloroplast ATPase. Proc. Natl. Acad. Sci. U.S.A. 84:3288-3292.

Pain, D., Kanwar, Y. S., and Blobel, G., 1988, Identification of a receptor for protein import into chloroplasts and its localization to envelope contact zones, Nature 311:232-237.

Palade, G. E., 1975, Intracellular aspects of the process of protein synthesis. Science 189:347358.

Palade, G. E., and Siekevitz, P., 1956a, Liver microsomes. An integrated morphological and biochemical study. J. Biophys. Biochem. Cytol. 2:171-200.

Palade, G. E., and Siekevitz, P., 1956b, Pancreatic microsomes. An integrated morphological and biochemical study. J. Biophys. Biochem. Cytol. 2:671-690. 
Palmiter, R. D., Gagnon, J., and Walsh, K. A., 1978, Ovalbumin, a secreted protein without a transient hydrophobic leader sequence. Proc. Natl. Acad. Sci. U.S.A. 75:94-98.

Palva, E. T., Hirst, T. R., Hardy, S. J. S., Holmgren, J., and Randall, L. L., 1981, Synthesis of a precursor of the B subunit of heat-labile enterotoxin in Escherichia coli. J. Bacteriol. 146:325330.

Parimoo, S., Rao, N., and Padmanaban, C., 1982, Cytochrome $c$ oxidase is preferably synthesized in the rough endoplasmic reticulum-mitochondria complex in rat liver. Biochem. J. 208:505507.

Park, K. S., Frost, B., Tuck, M., Ho, L. L., Kim, S., and Paik, W. K., 1987, Enzymatic metabolism of in vitro synthesized apocytochrome $\mathrm{c}$ enhances its transport into mitochondria, J. Biol. Chem. 262:14702-14708.

Park, S., Liu, G., Topping, T. B., Cover, W. H., and Randall, L. L., 1988, Modulation of folding pathways of exported proteins by the leader sequence, Science 239:1033-1035.

Paul, D. L., and Goodenough, D. A., 1983, In vitro synthesis and membrane insertion of bovine MP 26, an integral protein from lens fiber plasma membrane. J. Cell Biol. 96:633-638.

Perara, E. and Lingappa, R., 1986, A former aminoterminal signal sequence engineered to an internal location directs translocation of both flanking protein domains. J. Cell Biol. 101:22922301.

Perara, E., Rothman, R. E., and Lingappa, R., 1986, Uncoupling translocation from translation implication for transport of proteins across membrane. Science 232:348-352.

Perlman, D., and Halvorson, H. O., 1983, A putative signal peptidase recognition site and sequence in eukaryotic and prokaryotic signal peptides. J. Mol. Biol. 167:633-638.

Pfaller, R., and Neupert, W., 1987, High affinity binding sites involved in the import of porin into mitochondria. EMBO J. 6:2635-2642.

Pfanner, N., and Neupert, W., 1985, Transport of proteins into mitochondria: a potassium diffusion potential is able to drive the import of ADP/ATP carrier. EMBO J. 4:2819-2825.

Pfanner, N., and Neupert, W., 1986, Transport of $F_{1}$-ATPase subunit $\beta$ into mitochodria depends on both a membrane potential and nucleotide triphosphates. FEBS Lett. 209:152-156.

Pfanner, N., and Neupert, W., 1987, Distinct steps in the import of ADP/ATP carrier into mitochondria. J. Biol. Chem. 262:7528-7536.

Pfanner, N., Tropschug, M., and Neupert, W., 1987a, Mitochondrial protein import: Nucleotide triphosphates are involved in conferring import competence to precursors. Cell 49:815-823.

Pfanner, N., Hartl., F. U., Bernard, G., and Neupert, W., 1987b, Mitochondrial precursor proteins are imported through a hydrophilic membrane environment, Eur. J. Biochem. 169:289293.

Pfanner, N., Hoeben, P., Tropschug, M., and Neupert, W., 1987c, The carboxyl-terminal twothird of the ADP/ATP carrier polypeptide contains sufficient information to direct translocation into mitochondria, J. Biol. Chem. 262:14851-14854.

Pfanner, N., Müller, H. K., Harmey, M. A., and Neupert, W., 1987d, Mitochondrial protein import: involvement of the mature part of a cleavable precursor protein in the binding to receptor sites, EMBO J. 6:3449-3454.

Pfanner, N., Pfaller, R., Kleene, R., Ito, M., Tropschug, M., and Neupert, W., 1988, Role of ATP in mitochondrial protein import. Conformational alteration of a precursor protein can substitute for ATP requirement, J. Biol. Chem. 263:4049-4051.

Pfisterer, J., Lachman, P., and Kloppstech, K., 1982, Transport of proteins into chloroplasts. Binding of nuclear-coded chloroplast proteins to the chloroplast envelope. Eur. J. Biochem. 126: $143-148$.

Pilon, M., Jordi, W., De Kruijff, B., and Domel, R. A., 1987, Interaction of mitochondrial precursor protein apocytochrome $\mathrm{c}$ with phosphatidylserine in model membranes, Biochim. 
Biophys. Acta 902:207-216.

Pollit, S., and Zalkin, H., 1983, Role of primary structure and disulfide bound formation in $\beta$ lactamase secretion. J. Bacteriol. 153:27-32.

Poulis, M. I., Sham, D. C., Campbell, H. D., and Young, I. G., 1981, In vitro synthesis of respiratory NADH dehydrogenase of Escherichia coli. Role of UUG as initiation codon. Biochemistry 20:4178-4185.

Prehn, S., Tsamaloukas, A., and Rapoport, T. A., 1980, Demonstration of specific receptors of the rough endoplasmic membrane for the signal sequence of carp preproinsulin. Eur. J. Biochem. 107:185-195.

Prehn, S., Nürnberg, P., and Rapoport, T. A., 1981, A receptor for signal segments of secretory proteins in rough endoplasmic reticulum membrane. FEBS Lett. 123:79-84.

Prehn, S., Wiedmann, M., Rapoport, T. A., and Zwieb, C., 1987, Protein translocation across wheat germ microsomal membranes requires an SRP-like component. EMBO J. 6:2093-2097.

Rachubinski, R. A., Verma, D. P. S., and Bergeron, J. J. M., 1980, Synthesis of rat liver microsomal cytochrome $b_{5}$ by free ribosomes. J. Cell Biol. 85:705-716.

Racker, E., 1979, Reconstitution of membrane processes, in Methods in Enzymology (S. P. Colowick and D. Kaplan, eds.), Vol. 55, pp. 699-711, Academic Press, New York.

Randall, L. L., 1983, Translocation of domains of nascent periplasmic proteins across the cytoplasmic membrane is independent of elongation. Cell 33:231-240.

Randall, L. L., and Hardy, S. J. S., 1977, Synthesis of exported proteins by membrane bound polysomes from Escherichia coli. Eur. J. Biochem. 75:43-53.

Randall, L. L., and Hardy, S. J. S., 1986, Correlation of competence for export with lack of tertiary structure of the mature species: A study in vivo of maltose-binding protein in $E$. coli. Cell 46:921-928.

Randall, L. L., Hardy, S. J. S., and Josefsson, L. G., 1978, Precursors of three exported proteins in Escherichia coli. Proc. Natl. Acad. Sci. U.S.A. 75:1209-1212.

Rasmussen, B. A., and Bassford, P. J. Jr., 1985, Both linked and unlinked mutations can alter the intracellular site of synthesis of exported proteins of Escherichia coli. J. Bacteriol. 161:258264.

Rasmussen, B. A., and Silhavy, T. J., 1987, The first 28 amino acids of mature lam B are required for rapid and efficient export from the cytoplasm, Genes Dev. 1: 185-196.

Redman, C. M., and Sabatini, D. D., 1966, Vectorial discharge of peptides released by puromycin from attached ribosomes. Proc. Natl. Acad. Sci. U.S.A. 56:608-615.

Rees-Jones, R., and Alawqati, O., 1984, Proton-translocating adenosine triphosphatase in rough and smooth microsomes from rat liver. Biochemistry 23:2236-2240.

Reid, G. A., and Schatz, G., 1982, Import of proteins into mitochondria. Extramitochondrial pool and post-translational import of mitochondrial proteins precursors in vivo. J. Biol. Chem. 257:13062-13067.

Reid, G. A., Yonetani, T., and Schatz, G., 1982, Import of proteins into mitochondria. Import and maturation of mitochondrial intermembrane space enzymes cytochrome $b_{2}$ and cytochrome $c$ peroxidase in intact yeast cells. J. Biol. Chem. 257:13068-13074.

Reiss, B., Wasmann, C. C., and Bohnert, H. J., 1987, Regions in the transit peptide of SSU essential for transport into chloroplasts, Mol. Gen. Genet. 209:116-121.

Rhoads, D. B., Tai, P. C., and Davis, B. D., 1984, Energy requiring translocation of OmpA protein and alkaline phosphatase of Escherichia coli into inner membrane vesicle. J. Bacteriol. 159:63-70.

Richter, J. D., Lorenz, L. J., and Andet, R. G., 1985, Membrane-bound mRNAs are recruited from preinitiated ribonucleoprotein particles in injected Xenopus oocytes. J. Biol. Chem. 260:4448-4454. 
Rietveld, A., Jordi, W., and De Kruijff, B., 1986, Studies on the lipid dependency and mechanism of the translocation of the mitochondrial precursor protein apocytochrome $c$ across model membranes. J. Biol. Chem. 261:3846-3856.

Riezman, H., 1982, Binding of precursors of cytoplasmically-synthesized mitochondrial proteins to isolated outer membranes of yeast mitochondria. Prog. Clin. Res. 102b:161-170.

Riezman, H., Weir, E. M., Leaver, C. J., Titus, D. E., and Becker, W. M., 1980. Regulation of glyoxysomal enzymes during germination of cucumber. Plant Physiol. 65:40-46.

Riezman, H., Hay, R., Witte, C., Nelson, N., and Schatz, G., 1983a, Yeast mitochondrial outer membrane specifically binds cytoplasmically synthesized precursors of mitochondrial proteins. EMBO J. 2:1113-1118.

Riezman, H., Hase, T., Van Loon, A. P. G. M., Grivell, L. A., Suda, K., and Schatz, G., 1983b, Import of proteins into mitochondria: A 70-kilodalton outer membrane protein with a large carboxy-terminal deletion is still transported to the outer membrane. EMBO J. 2:21612168.

Riggs, P. D., Derman, A. I., and Beckwith, J., 1988, A mutation affecting the regulation of a sec A-lac $\mathrm{Z}$ fusion defines a new sec gene, Genetics 118:1571-579.

Rizzolo, L. J., Finidori, J., Gonzalez, A., Arpin, M., Ivanov, I. E., Adesnik, M., and Sabatini, D. D., 1985, Biosynthesis and intracellular sorting of growth hormone-viral envelope glycoprotein hybrids. J. Cell Biol. 101:1351-1356.

Roa, M., and Blobel, G., 1983, Biosynthesis of peroxisomal enzymes in the methylotropic yeast Hansenula polymorpha. Proc. Natl. Acad. Sci. U.S.A. 80:6872-6876.

Robbi, M., and Lazarow, P. B., 1978, Synthesis of catalase in two cell free protein synthesizing systems in rat liver. Proc. Natl. Acad. Sci. U.S.A. 75:4344-4348.

Robinson, A., Kaderbhai, M. A., and Austen, B. M., 1987, Identification of signal binding proteins integrated into the rough endoplasmic reticulum membrane. Biochem. J. 242:767-777.

Rodriguez-Garcia, M. F., and Sievers, A., 1977, Membrane contacts of the endoplasmic reticulum with plastids and with the plasmalemma in the endothecium of Scilla non-scripta. Cytobiologie 15:85-95.

Roggenkamp, R., Janiwicz, Z., Stanikowski, B., and Hollenberg, C. P., 1984, Biosynthesis and regulation of the peroxisomal methanol oxidase from methylotropic yeast Hansenula polymorpha. Mol. Gen. Genet. 194:489-493.

Roise, D., Horvath, S. J., Tomich, J. M., Richards, J. H., and Schatz, G., 1986, A chemically synthesized pre-sequence of an imported mitochondrial protein can form an amphiphilic helix and perturb natural and artificial phospholipid bilayers. EMBO J. 5:1327-1334.

Roise, D., Theiler, F., Horvath, S., Tomich, J. M., Richards, J. H., Allison, D. S., and Schatz, C., 1988, Amphiphilicity is essential for mitochondrial presequence function, EMBO J. 7:649653.

Rose, J. K., and Bergman, J. E., 1982, Expression from cloned cDNA of cell surface and secreted forms of the glycoprotein of vesicular stomatitis virus in eukaryotic cells. Cell 30:753-762.

Rosenblatt, M., Habener, J. F., Tyler, F. A., Shepard, G. L., and Potts, J. T. Jr., 1979, Chemical synthesis of precursor-specific region of pre-proparathyroid hormone. J. Biol. Chem. 254:14141421.

Rosenblatt, M., Beaudett, N. V., and Fasman, G. D., 1980, Conformational studies on the synthetic precursor specific region of pre-proparathyroid hormone. Proc. Natl. Acad. Sci. U.S.A. 77:3983-3987.

Rosenkrantz, A., Alam, T., Kim, K. S., Clark, B., Srere, P. A., Garante, L. P., 1986, Mitochondrial and nonmitochondrial citrate synthases in Saccharomyces cerevisiae are encoded by distinct homologous genes, Mol. Cell. Biol. 6:4509-4515.

Rothblatt, J. A., and Meyer, D. I., 1986a, Secretion in yeast: Reconstitution of translocation and glycosylation of $\alpha$-factor and invertase in homolgous cell free systems. Cell 44:619-628. 
Rothblatt, J. A., and Meyer, D. I., 1986b, Secretion in yeast: Translocation and glycosylation of pre-pro $\alpha$-factor in vitro can occur via an ATP dependent post-translational mechanism. $E M B O$ J. 5:1031-1036.

Rothblatt, J. A., Webb, J. R., Ammerer, G., and Meyer, D. I., 1987, Secretion in yeast: structural features influencing the post-translational translocation of prepro $\alpha$-factor in vitro, EMBO J. 6:3455-3468.

Rothman, J. E., and Lenard, J., 1977, Membrane asymmetry, the nature of membrane asymmetry provides clues to the puzzle of how membranes are assembled. Science 195:743-753.

Rothstein, S. J., Lazarus, C. M., Smith, W. E., Baulcombe, D. C., and Gatenby, A. A., 1984, Secretion of a wheat $\alpha$-amylase expressed in yeast. Nature (London) 308:662-665.

Rothstein, S. J., Gatenby, A. A., Wiley, D. L., and Gray, J. C., 1985, Binding of pea cytochrome $f$ to the inner membrane of Escherichia coli requires the bacterial sec A gene product. Proc. Natl. Acad. Sci. U.S.A. 82:7955-7959.

Rottier, P., Armstrong, J., and Meyer, D. I., 1985, Signal recognition particle-dependent insertion of coronavirus $\mathrm{E}_{1}$, an intracellular membrane glycoprotein. J. Biol. Chem. 260:46484652.

Russel, M., and Model, P., 1981, A mutant downstream from the signal peptidase cleaving site affects cleavage but not membrane insertion of phage coat protein. Proc. Natl. Acad. Sci. U.S.A. 78:1717-1721.

Ryan, J. P., Duncan, M. C., Bankaitis, Y. A., and Bassford, P. J. Jr., 1986, Intragenic reversion mutations that improve export of maltose-binding protein in Escherichia coli mal E signal sequence mutants. J. Biol. Chem. 261:3389-3395.

Rzepecki, L. M., Strittmatter, P., and Herbette, L. G., 1986, X-ray diffraction analysis of cytochrome $b_{5}$ reconstituted in egg phosphatidylcholine vesicles, Biophys. J. 49:829-838.

Sakaguchi, M., Mihara, K., and Sato, R., 1984, Signal recognition particle is required for cotranslational insertion of cytochrome P-450 into microsomal membrane. Proc. Natl. Acad. Sci. U.S.A. 81:3361-3364.

Sakaguchi, M., Mihara, K., and Sato, R., 1987, A short amino terminal segment of microsomal cytochrome P-450 functions both as an insertion signal and as a stop-transfer sequence. EMBO J. 6:2425-2431.

Sakakibara, R., Horio, Y., Ishiguro, M., Kanagawa, K., Matsuo, H., and Wada, H., 1987, An antibody and anti-idiotypic antibody against the extra signal peptide of pre-aspartate aminotransferase, Biochem. Biophys. Res. Commun. 148:979-988.

Sakakibara, R., Kamisaki, Y., Horio, Y., and Wada, H., 1983, Precursor of mitochondrial glutamic oxaloacetic transaminase isoenzyme exits as dimer. Biochem. Int. 6:231-238.

Santos, E., Kung, H., Young, I. G., and Kaback, H. R., 1982, In vitro synthesis of membrane bound $D$-lactate dehydrogenase of Escherichia coli. Biochemistry 21:2085-2091.

Sautter, C., Keller, G., and Hock, B., 1988, Glyoxysomal citrate synthase from watermelon cotyledons: immunocytochemical localization and heterologous translation in Xenopus oocytes, Planta 173:289-297.

Schatz, G., 1979, How mitochondria import proteins from the cytoplasm. FEBS Lett. 103:203211.

Schatz, G., and Butow, R. A., 1983, How are proteins imported into mitochondria. Cell 32:316318.

Schechter, I., Burstein, Y., Zemell, R., Ziv, E., Kantor, F., and Papermaster, D. S., 1979, Messenger RNA for opsin from bovine retina: Isolation and partial sequence of the in vitro translation product. Proc. Natl. Acad. Sci. U.S.A. 76:2654-2658.

Scheele, G. A., Jacoby, R., and Carne, T., 1980, Mechanism of compartmentation of secretory proteins: Transport of exocrine pancreatic proteins across the microsomal membrane. J. Cell Biol. 87:611-628. 
Schleyer, M., and Neupert, W., 1985, Transport of proteins into mitochondria: Translational intermediates spanning contact sites between outer and inner membranes. Cell 43:339-350.

Schleyer, M., Schmidt, B., and Neupert, W., 1982, Requirements of a membrane potential for the post-translational transfer of proteins into mitochondria. Eur. J. Biochem. 125:109-116.

Schmidt, B., Henning, B., Zimmermann, R., and Neupert, W., 1983, Biosynthetic pathway of mitochondrial ATPase subunit 9 in Neuropsora crassa. J. Cell Biol. 96:248-255.

Schmidt, G. W., Devillers-Thiery, A., Desruisseaux, H., Blobel, G., and Chua, N. H., 1979, Amino terminal amino acid sequences of precursor and mature forms of the ribulose 1,5bisphosphate carboxylase small subunit from Chlamydomonas reinhardtii. J. Cell Biol. 83:615623.

Schmidt, R. J., Gillham, N. W., and Boynton, J. E., 1985, Processing of the precursor to a chloroplast ribosomal protein made in the cytosol occurs in two steps, one of which depends on a protein made in the chloroplast. Mol. Cell. Biol. 5:1093-1099.

Schreier, P., Seftor, E. A., Schell, J., and Bohnert, H. J., 1985, The use of nuclear-encoded sequences to direct the light-regulated synthesis and transport of a foreign protein into plant chloroplasts. EMBO J. 4:25-32.

Schwaiger, M., Herzog, V., and Neupert, W., 1987, Characterization of translocation contact sites involved in the import of mitochondrial proteins. J. Cell Biol. 105:235-246.

Scotto, A. W., Goodwyn, D., and Zakim, D., 1987, Reconstitution of membrane proteins: Sequential incorporation of integral membrane proteins into preformed lipid bilayers. Biochemistry 26:833-839.

Scoulica, E., Krause, E., Meese, K., and Dobberstein, B., 1987, Disassembly and domain structure of the proteins in the signal recognition particle. Eur. J. Biochem. 163:519-528.

Seeburg, P. H., Shine, J., Martial, J. A., Ivarie, R. D., Morris, J. A., Ullrich, A., Baxter, J. D., and Goodman, H. M., 1978, Synthesis of growth hormone by bacteria. Nature (London) 276:795-798.

Seehara, J. S., and Khorana, H. G., 1984, Bacteriorhodopsin precursor characterization and its integration into the purple membrane. J. Biol. Chem. 259:4187-4193.

Sekizawa, J., Inouye, S., Halegoua, S., and Inouye, M., 1977, Precursors of major outer membrane proteins of Escherichia coli. Biochem. Biophys. Res. Commun. 77:1126-1133.

Severina, I. I., and Skulachev, V. P., 1984, Ethylrhodamine as a fluorescent penetrating cation and a membrane potential sensitive probe in cyanobacterial cells. FEBS Lett. 165:61-71.

Sharma, C. P., and Gehring, H., 1986, The precursor of mitochondrial aspartate aminotransferase is translocated into mitochondria as apoprotein. J. Biol. Chem. 261:11146-11149.

Shiba, K., Ito, K., Yura, T., and Cerretti, D. P., 1984, A defined mutation in the protein export gene within the spc ribosomal protein operon of Escherichia coli: Isolation and characterization of a new temperature-sensitive sec Y mutant. EMBO J. 3:631-635.

Shiba, K., Ito, K., and Yura, T., 1986, Suppressors of the sec Y 24 mutation: Identification and characterization of additional ssy genes in Escherichia coli. J. Bacteriol. 166:849-856.

Sidhu, A., and Beattie, D., 1983, Kinetics of assembly of complex III into the yeast mitochondrial membrane. Evidence for a precursor to the iron-sulfur protein. J. Biol. Chem. 256:1064910656.

Siegel, V., and Walter, P., 1985, Elongation arrest is not a prerequisite for secretory protein translocation across the microsomal membrane. J'. Cell Biol. 100:1913-1921.

Siegel, V., and Walter, P., 1986, Removal of the Alu structural domain from signal recognition particle leaves its protein translocation activity intact. Nature (London) 320:81-84.

Siegel, V., and Walter, P., 1988a, Each of the activities of signal recognition particle (SRP) is contained within a distinct domain: Analysis of biochemical mutants of SRP, Cell 52:39-49.

Siegel, V., and Walter, P., 1988b, The affinity of signal recognition particle for presecretory 
proteins is dependent on nascent chain length, EMBO J. 7:1769-1775.

Simon, K., Perara, E., and Lingappa, V. R., 1987, Translocation of globin fusion proteins across the endoplasmic reticulum membrane in Xenopus laevis oocytes. J. Cell Biol. 104:11651172.

Singer, S. J., 1977, The fluid mosaic model of membrane structure, in Structure of Biological Membranes (S. Abrahamsson and I. Pascher, eds.), pp. 443-461, Plenum Press, New York.

Singer, S. J., Maher, P. A., and Yaffe, M., 1987a, On the translocation of proteins across membranes. Proc. Natl. Acad. Sci. U.S.A. 84:1015-1019.

Singer, S. J., Maher, P. A., and Yaffe, M. P., 1987b, On the transfer of integral proteins into membranes. Proc. Natl. Acad. Sci. U.S.A. 84:1960-1964.

Skerjanc, I. S., Shore, G. C., and Silvius, J. R., 1987, The interaction of a synthetic mitochondrial signal peptide with lipid membranes is independent of transbilayer potential, EMBO J. 6:31173123.

Small, G. M., and Lazarow, P. B., 1987, Import of the carboxy-terminal portion of acyl-CoA oxidase into peroxisomes of Candida tropicalis. J. Cell Biol. 105:247-250.

Smeekens, S., Bauerle, C., Hageman, J., Keegstra, K., and Wasbeek, P., 1986, The role of the transit peptide in the routing of precursors towards different chloroplast comparttments. Cell 46:365-375.

Smeekens, S., van Steeg, H., Bauerle, C., Bettenbroek, H., Keegstra, K., and Weisbeek, P., 1987, Import into chloroplasts of a yeast mitochondrial protein directed by ferredoxin and plastocyanin transit peptides, Plant Mol. Biol. 9:377-388.

Smith, H., Bron, S., Van Ee, J., and Venema, G., 1987, Construction and use of signal sequence selection vectors in Escherichia coli and Bacillus subtilis. J. Bacteriol. 169:3321-3328.

Smith, W. P., 1980, Co-translational secretion of diphtheria toxin and alkaline phosphatase in vitro: Involvement of membrane protein(s). J. Bacteriol. 141:1142-1147.

Smith, W. P., Tai, P. C., and Davis, B. D., 1978a, Nascent peptides as sole attachment of polysomes to membranes in bacteria. Proc. Natl. Acad. Sci. U.S.A. 75:814-817.

Smith, W. P., Tai, P. C., and Davis, B. D., 1978b, Interaction of secreted nascent chains with surrounding membrane in Bacillus subtilis. Proc. Natl. Acad. Sci. U.S.A. 75:5921-5925.

Smith, W. P., Tai, P. C., and Davis, B. D., 1979, Extracellular labeling of growing secreted polypeptide chains with surrounding membrane with diazoiodosulfanilic acid. Biochemistry 18:198-202.

Smith, W. P., Tai, P. C., and Davis, B. D., 1981, Bacillus licheniformis penicillinase: Cleavage and attachment of lipid during co-translational secretion. Proc. Natl. Acad. Sci. U.S.A. 78:35013505.

Soellner, T., Pfanner, N., and Neupert, W., 1988, Mitochondrial protein import: differential recognition of various transport intermediates by antibodies, FEBS. Lett. 229:25-29.

Spiess, M., and Lodish, H. F., 1985, The sequene of a second human asialoglycoprotein receptor: Conservation of two receptors during evolution. Proc. Natl. Acad. Sci. U.S.A. 82:6465-6469.

Spiess, M., and Lodish, H. F., 1986, An internal signal sequence; the asialoglycoprotein receptor membrane anchor is necessary. Cell 44:177-185.

Spiess, M., and Handschin, C., 1987, Deletion analysis of the internal signal-anchor domain of the human asialoglycoprotein receptor H. EMBO J. 6:2683-2691.

Spiess, M., Schwartz, A. L., and Lodish, H. F., 1985, Sequence of human asialoglycoprotein receptor cDNA, an internal signal sequence for membrane insertion. J. Biol. Chem. 260:19791982.

Strauch, K. L., Kumamoto, C. A., and Beckwith, J., 1986, Does sec A mediate coupling between secretion and translation in Escherichia coli? J. Bacteriol. 166:505-512.

Strittmatter, P., Thiede, M. A., Hackett, C. S., and Ozols, J., 1988, Bacterial synthesis of active 
rat stearyl-CoA desaturase lacking the 26-residue amino-terminal amino acid sequence, $J$. Biol. Chem. 262:2532-2535.

Strom, M. S., and Lory, S., 1987, Mapping of export signals of Pseudomonas aeruginosa pili with alkaline phosphatase fusions. J. Bacteriol. 169:3181-3188.

Strubin, M., Mach, B., and Long, E. O., 1984, The complete sequence of the mRNA for HLADR associated invariant chain reveals a polypeptide chain with an unusual transmembrane polarity. EMBO J. 3:869-872.

Stuart, R. A., Neupert, W., and Tropschug, M., 1987, Deficiency in mRNA splicing in a cytochrome $c$ mutant of Neurospora crassa: Importance of carboxy terminus for import of apocytochrome $c$ into mitochondria. EMBO J. 6:2131-2137.

Sugimoto, K., Sugisaki, H., Okamoto, T., and Takanami, M., 1977, Studies on the bacteriophage fd DNA. IV. The sequence of messenger RNA for the major coat protein gene. J. Mol. Biol. 111:487-507.

Sugita, H., Tobe, T., Sakamoto, T., and Higashi, T., 1982, Immature precursor catalase in subcellular fractions of rat liver. J. Biochem. (Tokyo) 92:509-515.

Suissa, M., and Schatz, G., 1982, Import of proteins into mitochondria. Translatable mRNA for imported mitochondrial proteins are present in free as well as mitochondrial-bound cytoplasmic polysomes. J. Biol. Chem. 257:13048-13055.

Sutcliffe, J. G., 1978, Nucleotide sequence of the ampicillin resistance gene of Escherichia coli plasmide PBR 322. Proc. Natl. Acad. Sci. U.S.A. 75:3737-3741.

Suzuki, Y., Orii, T., Takiguchi, M., Mori, M., Hijikata, M., and Hashimoto, T., 1987, Biosynthesis of membrane polypeptides of rat liver peroxisomes. J. Biochem. 101:491-496.

Sveda, M. M., Markoff, L. J., and Lai, C. J., 1982, Cell surface expression of the influenza virus hemagglutinin requires the hydrophobic carboxy terminal sequences. Cell 30:649-656.

Swinkels, B. W., Evers, R., and Borst, P., 1988, The topogenic signal of glycosomal (microbody) phosphoglycerate kinase of Crithidia fasciaculata resides in a carboxy-terminal extension, EMBO J. 7:1159-1165.

Sztul, E. S., Hendrick, J. P., Kraus, J. P., Wall, D., Kalousek, F., and Rosenberg, L. E., 1987, Import of rat ornithine transcarbamylase precursor into mitochondria: two-step processing of the leader peptide, J. Cell. Biol. 105:2631-2639.

Tabe, L., Krieg, P., Strachan, R., Jackson, D., Wallis, E., and Colman, A., 1984, Segregation of mutant ovalbumins and ovalbumin-globin proteins in Xenopus oocytes. J. Mol. Biol. 180:645666.

Tadashi, J., Hisayuki, N., Shuichiro, M., Kazunori, S., and Yoshimasa, M., 1985, Cloning and sequence analysis of a cDNA encoding porcine mitochondrial aspartate aminotransferase precursor, Proc. Natl. Acad. Sci. USA 82:6065-6069.

Tajima, S., Lauffer, L., Rath, V. L., and Walter, P., 1986, The signal recognition particle receptor is a complex that contains two distinct polypeptide chains. J. Cell Biol. 103:1167-1178.

Takagaki, Y., Radhakrishnan, R., Gupta, C. M., and Khorana, H. G., 1983a, The membrane embedded segment of cytochrome $b_{5}$ as studied by cross-linking with photoactivable phospholipids. I. Transferable form. J. Biol. Chem. 258:9128-9135.

Takagaki, Y., Radhakrishnan, R., Wirtz, K. W. A., and Khorana, H. G., 1983b, The membrane embedded segment of cytochrome $b_{5}$ as studied by cross-linking with photoactivable phospholipids. II. Nontransferable form. J. Biol. Chem. 258:9136-9143.

Takiguchi, M., Miura, S., Mori, M., and Tatibana, M., 1983, Transport of proteins into mitochondria: A high conservation of precursor uptake and processing systems. Comp. Biochem. Physiol. B 75B:227-231.

Taldmage, K., Stahl, S., and Gilbert, W., 1980a, Eukaryotic signal sequence transports insulin antigen in Escherichia coli. Proc. Natl. Acad. Sci. U.S.A. 77:3369-3373. 
Talmadge, K., Kaufman, S., and Gilbert, W., 1980b, Bacteria mature preproinsulin to proinsulin. Proc. Natl. Acad. Sci. U.S.A. 77:3988-3992.

Taldmadge, K., Brosius, J., and Gilbert, W., 1981, An "internal" signal sequence directs secretion and processing of proinsulin in bacteria. Nature (London) 294:176-178.

Teintze, M., Slaughter, M., Weiss, H., and Neupert, W., 1982, Biogenesis of mitochondrial ubiquinol: Cytochrome creductase (cytochrome $b c_{1}$ complex). Precursor proteins and their transfer into mitochondria. J. Biol. Chem. 257:10364-10371.

Thiede, M. A., Ozols, J., and Strittmatter, P., 1986, Construction and sequence of cDNA for rat liver stearyl coenzyme A desaturase. J. Biol. Chem. 261:13230-13235.

Thibodeau, S. N., Lee, D. C., and Palmiter, R. D., 1978, Identical precursors for serum transferrin and egg white conalbumin. J. Biol. Chem. 253:3771-3774.

Turn, N. J., and Silhavy, T. J., 1987, Characterization and in vivo cloning of prl C, a suppressor of signal sequence mutation in Escherichia coli, Genetics 116:513-521.

Tweten, R. K., and Iandolo, J. J., 1983, Transport and processing of staphylococcal enterotoxin B. J. Bacteriol. 153:297-303.

Valusek, G. P., Inouye, S., and Inouye, M., 1984, Effects of replacing serine and threonine residues within the signal peptide on the secretion of the major outer membrane lipoprotein of Escherichia coli. J. Biol. Chem. 259:6195-6200.

Van Deenen, L. L. M., 1981, Topology and dynamics of phosopholipids in membranes, FEBS Lett. 123:3-15.

Van Den Broek, G., Timko, M. P., Kausch, A. P., Cashmore, A. R., Montagu, M. V., and Herrera-Estrella, L., 1985, Targeting of a foreign protein to chloroplasts by fusion to the transit peptide from the small subunit of ribulose 1,5-bisphosphate carboxylase. Nature (London) 313:358-363.

Van Loon, A. P. G. M., and Schatz, G., 1987, Transport of proteins to mitochondrial intermembrane space: The "sorting" domain of cytochrome $c_{1}$ presequence is a stop-transfer sequence specific for mitochondrial inner membrane. ЕMBO J. 6:2441-2448.

Van Loon, A. P. G. M., and Young, E. T., 1986, Intracellular sorting of alcohol dehydrogenase isoenzymes in yeast: A cytosolic location reflects absence of an aminoterminal targeting sequence for the mitochondria. EMBO J. 5:161-165.

Van Loon, A. P. G. M., De Groot, R. J., De Haan, M., Dekker, A., and Grivell, L. A., 1984, The DNA sequence of the nuclear gene coding for the $17 \mathrm{kd}$ subunit VI of the yeast ubiquinol-cytochrome $c$ reductase: A protein with an extremely high content of acidic amino acid. EMBO J. 3:1039-1043.

Van Loon, A. P. G. M., Brändli, A. W., and Schatz, G., 1986, The presequence of two imported mitochondrial proteins contains information for intracellular and intramitochondrial sorting. Cell 44:801-812.

Van Loon, A. P. G. M., Brädli, A. W., Pesold-Hurt, B., Blank, D., and Schatz, G., 1987, Transport of proteins into the mitochondrial intermembrane space: "Matrix targeting" and the sorting domains in cytochrome $c_{1}$ presequence. EMBO J. 6:2433-2439.

Van Steeg, H., Oudshoorn, P., Van Hell, B., Polman, J. E. M., and Grivell, L. A., 1986, Targeting efficiency of a mitochondrial presequence is dependent on the passenger protein. EMBO J. 5:3643-3650.

Vassarotti, A., Chen, W. J., Smagula, C., and Douglas, M. G., 1987a, Sequences distal to mitochondrial targeting sequence are necessary for the maturation of $F_{1}$-ATPase $\beta$-subunit precursor in mitochondria. J. Biol. Chem. 262:411-418.

Vassarotti, A., Stroud, R., and Douglas, M., 1987b, Independent mutations at the amino terminus of a protein act as surrogate signals for mitochondrial import. EMBO J. 6:705-711.

Verner, K., and Schatz, G., 1987, Import of an incompletely folded precursor protein into isolated 
mitochondria requires an energized inner membrane, but no added ATP. EMBO J. 6:24492456.

Vestweber, D., and Schatz, G., 1988, Point mutations destabilizing a precursor protein enhance its post-translational import into mitochondria, EMBO J. 7:1147-1151.

Viebrock, A., Perz; A., and Sebald, W., 1982, The imported preprotein of the proteolipid subunit of the mitochondrial ATP synthase from Neurospora crassa. Molecular cloning and sequencing of the mRNA. EMBO J. 1:565-571.

Von Heijne, G., 1980, Transmembrane translocation of proteins. A detailed physicochemical analysis. Eur. J. Biochem. 103:431-438.

Von Heijne, G., 1984, How signal sequences maintain cleavage specificity. J. Mol. Biol. 173:243251.

Von Heijne, G., 1985, Signal sequences. The limits of variation. J. Mol. Biol. 134:99-105.

Von Heijne, G., 1986, Mitochondrial targeting sequences may form amphiphilic helices. EMBO J. 5:1335-1342.

Von Heijne, G., and Blomberg, C., 1979, Transmembrane translocation of proteins. The direct transfer model. Eur. J. Biochem. 97:175-181.

Von Heijne, G., and Segrest, J. P., 1987, The leader peptides from bacteriorhodopsin and halorhodopsin are potential membranes spanning amphiphilic helixes. FEBS Lett. 213:238-240.

Walk, R. A., and Hock, B., 1978, Cell-free synthesis of glyoxysomal malate dehydrogenase. Biochem. Biophys. Res. Commun. 81:636-645.

Walter, A., Margolis, D., Mohan, R., and Blumenthal, R., 1986, Apocytochrome $c$ induces pHdependent vesicle fusion. Membr. Biochem. 6:217-237.

Walter, P., and Blobel, G., 1980, Purification of a membrane-associated protein complex required for translocation across the endoplasmic reticulum. Proc. Natl. Acad. Sci. U.S.A. 77:71127116.

Walter, P., and Blobel, G., 1981a, Translocation of proteins across the endoplasmic reticulum. II. Signal recognition protein (SRP) mediates the selective binding to microsomal membranes of in vitro assembled polysomes synthesizing secretory proteins. J. Cell Biol. 91:551-556.

Walter, P., and Blobel, G., 1981b, Translocation of proteins across the endoplasmic reticulum. III. Signal recognition protein (SRP) causes signal sequence dependent and site specific arrest of chain elongation that is released by microsomal membranes. J. Cell Biol. 91:557-561.

Walter, P., and Blobel, G., 1983, Disassembly and reconstitution of signal recognition particle. Cell 34:525-533.

Walter, P., Jackson, R. C., Marcus, M. M., Lingappa, V. R., and Blobel, G., 1979, Tryptic dissection of receptor(s) for the translocation of presecretory proteins across the microsomal membrane and reconstitution of translocation activity. Proc. Natl. Acad. Sci. U.S.A. 76:17951799.

Walter, P., Ibrahimi, I., and Blobel, G., 1981, Translocation of proteins across the endoplasmic reticulum. I. Signal recognition protein (SRP) binds to in vitro assembled polysomes synthesizing secretory protein. J. Cell Biol. 91:545-551.

Wandinger-Ness, A. U., and Weiss, R. L., 1987, A single precursor protein for two separate mitochondrial enzymes in Neurospora crassa, J. Biol. Chem. 262:5823-5830.

Wanner, B. L., Sarthy, A., and Beckwith, J. R., 1979, Escherichia coli periplasmic mutant that reduces amounts of several periplasmic and outer membrane proteins. J. Bacteriol. 140:229239.

Warren, T. G., and Dobberstein, B., 1978, Protein transfer across microsomal membrane reassembled from separated membrane components, Nature 273:569-571.

Wasmann, C., C., Reis, B., Bartlett, S. G., and Bohnert, H., 1987, The importance of the transit peptide and the transported protein for import into chloroplasts, MGG. Mol. Gen. Genet. 205:445-453. 
Watanabe, K., and Kubo, S., 1982, Mitochondrial adenylate kinase from chicken liver. Purification, characterization and its cell-free synthesis. Eur. J. Biochem. 123:587-592.

Watanabe, M., Hunt, J. F., and Blobel, G., 1986, In vitro synthesized outer membrane protein is integrated into bacterial inner membrane but translocated across microsomal membranes. $\mathrm{Na}$ ture 323:71-73.

Waters, M. G., and Blobel, G., 1986, Secretory protein translocation in a yeast cell free system can occur post-translationally and requires ATP hydrolysis. J. Cell Biol. 102:1543-1550.

Waters, M. G., Chirico, W. J., and Blobel, G., 1986, Protein translocation across yeast microsomal membranes is stimulated by a soluble factor. J. Cell Biol. 103:2629-2636.

Watson, E. E., 1984, Compilation of published signal sequences. Nucleic Acids Res. 12:51455164.

Watts, C., Silver, P., and Wickner, W., 1981, Membrane assembly from purified components. II. Assembly of $\mathrm{M}_{13}$ procoat into liposomes reconstituted with purified leader peptides. Cell 25:347353.

Webster, K. A., Patel, H. V., Freeman, K. B., and Papahadjopoulos, D., 1979, Interaction of mitochondrial malate dehydrogenase monomer with phospholipid vesicles. Biochem. J. 178:147158.

White, J. A., and Scandalios, J. G., 1987, In vitro synthesis and processing of Mn-superoxide dismutase (SOD-3) into maize mitochondria, Biochim. Biophys. Acta 226:16-25.

Wickner, W., 1979, The assembly of proteins into biological membranes. The membrane trigger hypothesis. Annu. Rev. Biochem. 49:23-45.

Wiedmann, M., Huth, A., and Rapoport, T. A., 1986a, A signal sequence is required for the functions of the signal recognition particle. Biochem. Biophys. Res. Commun. 134:790796.

Wiedmann, M., Huth, A., and Rapoport, T. A., 1986b, Internally transposed signal sequence of carp preproinsulin retains its function with the signal recognition particle. FEBS Lett. 194:139145.

Wiedmann, M., Kurzchalia, T. V., Bielka, H., and Rapoport, T. A., 1987, Direct probing of the interaction between the signal sequence of nascent preprolactin and the signal recognition particle by specific cross-linking. J. Cell Biol. 104:201-208.

Wiedmann, M., Kurzchalia, T. V., Hartmann, E., and Rapoport, T. A., 1987, A signal sequence receptor in the endoplasmic reticulum membrane, Nature 328:830-833.

Wiedmann, M., Wiedmann, B., Voigt, S., Wachter, E., Müller, H. G., and Rapport, T. A., 1988, Post-translational transport of proteins into microsomal membranes of Candida maltosa, EMBO J. 7:1763-1768.

Wolfe, P. B., and Wickner, W., 1984, Bacterial leader peptidase, a membrane protein without a leader peptide uses the same export pathway as presecretory proteins, Cell 36:1067-1072.

Wolfe, P. B., Wickner, W., and Goodman, J. M., 1983, Sequence of the leader peptidase gene of Escherichia coli and the orientation of leader peptidase in the bacterial envelope. J. Biol. Chem. 258:12073-12080.

Wu, H. C., Hou, C., Lin, J. J. C., and Yem, D. W., 1977, Biochemical characterization of a mutant lipoprotein of Escherichia coli. Proc. Natl. Acad. Sci. U.S.A. 74:1388-1392.

Yaffe, M. P., and Schatz, G., 1984, Two nuclear mutations that block mitochondrial protein import in yeast. Proc. Natl. Acad. Sci. U.S.A. 81:4819-4823.

Yamaguchi, J., Mori, H., and Nishimura, M., 1987, Biosynthesis and intracellular transport of glyoxysomal malate dehydrogenase in germinating pumpkin cotyledons. FEBS Lett. 213:329332.

Yamaguchi, J., Nishimura, M., and Akazawa, T., 1986, Purification and characterization of hemecontaining low-affinity form of catalase from seeding cotyledons, Eur. J. Biochem. 159:315322. 
Yamamoto, K., and Fahimi, H. D., 1987, Three-dimensional reconstruction of a peroxisomal reticulum in regenerating rat liver. J. Cell Biol. 105:713-722.

Yamamoto, M., Hayashi, N., an Kikuchi, G., 1981, Regulation of synthesis and intracellular translocation of $\beta$-aminolevulinate synthase by heme and its relation to the heme saturation of tryptophan pyrolase in rat liver, Arch. Biochem Biophys. 209:451-459.

Yamane, K., Ichihara, S., and Mizushima, S., 1987, Transloction of protein across Escherichia coli membrane vesicles requires both the proton motive force and ATP. J. Biol. Chem. 262:2358-2362.

Yazgu, M., Shiota-Niiya, S., Shimamoto, T., Kanazawa, H., Futai, M., and Tsuchiya, T., 1984, Nucleotide sequence of mel B gene and characteristics of deduced amino acid sequence of melibiose carrier in Escherichia coli. J. Biol. Chem. 259:4320-4326.

Yoshida, Y., Hashimoto, T., Kimura, H., Sakakibara, S., and Tagawa, K., 1985, Interaction with mitochondrial membranes of a synthetic peptide with a sequence common to extra peptides of mitochondrial precursor protein. Biochem. Biophys. Res. Commun. 128:775-780.

Yost, S. C., Hedgpeth, J., and Lingappa, V. R., 1983, A stop transfer sequence confers predictable transmembrane orientation to a previously secreted protein in cell free systems. Cell 34:759-766.

Young, E. T., and Pilgrim, D., 1985, Isolation and DNA sequence of $\mathrm{ADH}_{3}$, a nuclear gene encoding the mitochondrial isoenzyme of alcohol dehydrogenase in Saccharomyces cerevisiae. Mol. Cell. Biol. 5:3024-3034.

Yu, L. M., Merchant, S., Theg, S. M., and Selman, B. R., 1988, Ioslation of cDNA clone for the $\gamma$ subunit of the chloroplast ATP synthase of Chlamydomonas reinhardtii: import and cleavage of the precursor, Proc. Natl. Acad. Sci. USA 85:1369-1373.

Zaar, K., Völkl, A., and Fahimi, H. D., 1986, Isolation and characterization of peroxisomes from the renal cortex of beef, sheep, and cat. Eur. J. Cell Biol. 40:16-24.

Zaar, K., Völkl, A., and Fahimi, H. D., 1987, Association of isolated bovine kidney cortex peroxisomes with endoplasmic reticulum. Biochim. Biophys. Acta 897:135-142.

Zerial, M., Melancon, P., Schneider, C., and Garoff, H., 1986, The transmembrane segment of the human transferrin receptor functions as a signal peptide. EMBO J. 5:1543-1550.

Zerial, M., Huylebroeck, D., and Garoff, H., 1987, Foreign transmembrane peptides replacing the internal signal sequences of transferrin receptor allow its translocation and membrane binding. Cell 48:147-155.

Zimmermann, R., and Neupert, W., 1980a, Transport of proteins into mitochondria. Post-translational transfer of ADP/ATP carrier into mitochondria in vitro. Eur. J. Biochem. 109:217229.

Zimmermann, R., and Neupert, W., 1980b, Biogenesis of glyoxysomes. Synthesis and intracellular transfer of isocitrate lyase. Eur. J. Biochem. 112:225-233.

Zimmermann, R., and Neupert, W., 1981, Different transport pathways of individual precursor proteins in mitochondria. Eur. J. Biochem. 116:455-460.

Zimmermann, R., and Wickner, W., 1983, Energetics and intermediates of the assembly of protein OmpA into the outer membrane of Escherichia coli. J. Biol. Chem. 258:3920-3925.

Zimmermann, R., Watts, C., and Wickner, W., 1982, The biosynthesis of membrane bound $\mathrm{M}_{13}$ coat protein. Energetics and assembly intermediates. J. Biol. Chem. 257:6529-6536.

Zwizinski, C., and Neupert, W., 1983, Precursor protẹins are transported into mitochondria in the absence of proteolytic cleavage of the additional sequences. J. Biol. Chem. 258:13340-13346.

Zwizinski, C., Schleyer, M., and Neupert, W., 1983, Transport of proteins into mitochondria. Precursor to the ADP/ATP carrier binds to receptor sites on isolated mitochondria. J. Biol. Chem. 258:4071-4074.

Zwizinski, C., Schleyer, M., and Neupert, W., 1984, Proteinaceous receptor for the import of mitochondrial precursor proteins. J. Biol. Chem. 259:7850-7856. 